\title{
Stereochemistry of Platinum Organometallics
}

\author{
Milan Melník*,a and Ján Garaj ${ }^{\mathrm{a}}$, and Clive Eduard Holloway ${ }^{\mathrm{b}}$ \\ ${ }^{a}$ Department of Natural Sciences, Alexander Dubcek University in Trencin, Studentska 1, 81250 Trencin, Slovak \\ Rebublic \\ ${ }^{b}$ Department of Chemistry, York University, 4700 Keele st., North York M 3J, 1P3, Ontario, Canada
}

\begin{abstract}
The organometallic chemistry of platinum covers a huge field, as shown by a recent survey covering the crystallographic and structural data of almost one thousand organometallic derivatives. About nine percent of those derivatives exist as isomers and are summarized in this review and include distortion (90\%), cis - trans (6\%) and ligand isomerism (4\%). These are discussed in terms of the coordination about the platinum atom, and correlations are drawn between donor atom, bond length and interbond angles, with attention to any trans - effect.

Distortion isomers, differing only by degree of distortion in Pt-L bond lengths and L- Pt -L bond angles, are the most numerous. They are also spread over a wider range of oxidation states of platinum, (zero, $+1,+2,+4$, mixed valence +1 plus +2 and +2 plus +4 ), compared to cis-trans and ligand isomerism in which only +2 is found.
\end{abstract}

Keywords: Pt, Stereochemistry, X-ray, Isomerisms, Distortion, Cis-, Trans-, Ligand.

\section{INTRODUCTIONS}

The organometallic chemistry of platinum covers a vast range of compounds as shown by a recent survey covering the crystallographic and structural data of almost one thousand examples [1-4]. Systematic studies in the field of stereoselectivity of complexes over the last 50 years have become of increasing interest since this is very often related to important stereospecificity of biological systems, and the catalysis and stereochemical effects in technical processes. Isomers can be broadly classed into two major categories, structural and stereoisomers. The former can be divided into: ionization, hydrate, coordination, linkage and polymerization sub-categories. The latter can be divided into geometric (cistrans, fac-mer), optical, ligand and distortion isomerism.

Recently [5] we analyzed and classified over two hundred isomeric examples of platinum monomeric coordination complexes about sixty five percent of which are distortion isomers, about $30 \%$ cis - trans and the remainder mixed isomers (cis-trans plus distortion) and ligand isomerism. Distortion isomers, differing only by degree of distortion in $\mathrm{Pt}-$ $\mathrm{L}$ distances and L-Pt-L angles, are by far the most numerous.

In this review we analyze and classify isomers of platinum organometallic complexes, to show that stereoisomers are more common than structural isomers, and, surprisingly, that distortion isomerism is again more common than the better known cis - trans isomerism. The aim of this manuscript is to discuss the factors which could lead to a better understanding of stereochemical interaction within the coordination sphere of platinum organometallic compounds, and to examine some cooperative effects between isomeric types.

The system has been classified according to the coordination number of the platinum, and further subdivided in each

*Address correspondence to this author at the Department of Natural Sciences, Alexander Dubcek University in Trencin, Studentska 1, 81250 Trencin, Slovak Rebublic; Tel: +421 2 52495239; Fax: +421 2 52493198; E-mail:milan.melnik@stuba.sk group into monomers and oligomers. The compounds have been listed and referenced in order of increasing coordination number, increasing complexity of the coordination sphere, and increasing atomic number of the principal coordinating ligand. Where the oxidation number for a platinum atom is not designated, it may be taken to be platinum(II). .

\section{DISTORTION ISOMERISM}

The coexistence of two or more species differing only by degree of distortion of M-L bond distances and L-M-L bond angles is typical of the general class of distortion isomers [6]. There are almost one hundred examples in the chemistry of platinum organometallic compounds. The platinum oxidation states in these isomers are found in the oxidation states of zero, $+1,+2$ (the most common) and +4 .

\subsection{Mononuclear Complexes}

\subsubsection{Isomeric Forms}

Crystallographic and structural data for mononuclear platinum organometallic compounds which exist in two [736] and even three [37] isomeric forms are gathered in Table 1. Colourless $\mathrm{Pt}\left(\mathrm{PPh}_{3}\right)_{3} \mathrm{CO}$, monoclinic [7] and rhombohedral [8] are the only examples of a pair of $\mathrm{Pt}(0)$ distortion isomers. The isomers consist of three bulky unidentate $\mathrm{PPh}_{3}$ ligands and $\mathrm{CO}$ ligand in an approximately tetrahedral geometry about each $\operatorname{Pt}(0)$ atom.

There are thirty six $\mathrm{Pt}(\mathrm{II})$ [10-34] and one Pt(IV) $[35,36]$ distortion isomers which exist in two isomeric forms with homo- as well as hetero- crystal classes and pale yellow $\left[\mathrm{Pt}\left(\eta-\mathrm{CH}_{2} \mathrm{Cl}\right)\left(\eta^{3}\right.\right.$-tmba)Cl] [37] exists in three monoclinic isomeric forms (Table $\mathbf{1}$ ).

In twelve derivatives both isomeric classes belong to the homo-monoclinic [11, 21-28], orthorhombic [18], triclinic $[19,20]$ and tetragonal[35,36]. The remaining examples differ from each other not only by degree of distortion but also by crystal class. In twelve of these one isomer is monoclinic and the other orthorhombic $[9,13,14,29-33]$. In two examples 
Table 1. Crystallographic and Structural Data for Mononuclear Platinum Organometallic Compounds - Distortion Isomers ${ }^{\mathrm{a}}$

\begin{tabular}{|c|c|c|c|c|c|c|c|c|c|}
\hline $\begin{array}{l}\text { COMPOUND } \\
\text { (colour) }\end{array}$ & $\begin{array}{c}\text { Cryst. cl. } \\
\text { SPACE GR. } \\
\text { Z }\end{array}$ & $\begin{array}{l}\mathbf{a}[\AA ̊ \\
\mathbf{b}[\AA] \\
\mathbf{c}[\AA ̊\end{array}$ & $\begin{array}{l}\alpha\left[^{\circ}\right] \\
\beta\left[^{\circ}\right] \\
\gamma\left[^{\circ}\right]\end{array}$ & $\begin{array}{l}\text { Chromo- } \\
\text { PHORE }\end{array}$ & & $\begin{array}{l}\mathbf{P t}-\mathbf{L} \\
{[\AA ̊}\end{array}$ & \multicolumn{2}{|c|}{$\begin{array}{c}\mathbf{L}-\mathbf{P t}-\mathbf{L} \\
{\left[{ }^{\circ}\right]}\end{array}$} & Ref. \\
\hline $\begin{array}{c}\mathbf{P t}^{0}\left(\mathbf{P P h}_{3}\right)_{3}(\mathbf{C O}) \\
(\text { colourless })\end{array}$ & $\begin{array}{c}\mathrm{m} \\
\mathrm{P} 2_{1} / \mathrm{n} \\
4\end{array}$ & $\begin{array}{l}11.275(5) \\
30.904(8) \\
13.298(5)\end{array}$ & $91.6(1)$ & $\mathrm{PtP}_{3} \mathrm{C}$ & $\begin{array}{c}\mathrm{Ph}_{3} \mathrm{P}^{\mathrm{b}} \\
\mathrm{OC}\end{array}$ & $\begin{array}{c}2.334(8,1) \\
1.86(3)\end{array}$ & $\begin{array}{l}\mathrm{P}, \mathrm{P}^{\mathrm{b}} \\
\mathrm{P}, \mathrm{C}\end{array}$ & $\begin{array}{c}111.4(3,2.3) \\
107(1,2)\end{array}$ & [7] \\
\hline $\begin{array}{c}\text { cis- } \mathrm{Pt}(\mathrm{Me})_{2}(\mathrm{MeNC})_{2} \\
(\text { colourless })\end{array}$ & $\begin{array}{c}\mathrm{m} \\
\mathrm{C} 2 / \mathrm{c} \\
8\end{array}$ & $\begin{array}{c}26.584(2) \\
6.062(1) \\
11.318(1)\end{array}$ & $108.038(7)$ & $\mathrm{PtC}_{4}$ & $\begin{array}{c}\mathrm{MeC} \\
\mathrm{MeNC}\end{array}$ & $\begin{array}{c}2.065(30,5) \\
1.99(2,3)\end{array}$ & $\mathrm{C}, \mathrm{C}$ & $\begin{array}{l}90.0(9,5.3) \\
174.2(9,9)\end{array}$ & [9] \\
\hline $\begin{array}{c}\mathrm{Pt}\left(\eta^{2}-\mathrm{C}_{8} \mathrm{H}_{4} \mathrm{O}_{2}\right)\left(\mathrm{PPh}_{3}\right)_{2} \\
\text { (red) }\end{array}$ & $\begin{array}{c}\mathrm{m} \\
\mathrm{P} 2{ }_{1} / \mathrm{c} \\
4\end{array}$ & $\begin{array}{l}11.79 \\
22.27 \\
15.64\end{array}$ & 122.0 & $\mathrm{PtC}_{2} \mathrm{P}_{2}$ & & & & & [10] \\
\hline $\begin{array}{c}\mathrm{Pt}\left(\eta^{2}-\mathrm{C}_{8} \mathrm{H}_{4} \mathrm{O}_{2}\right)\left(\mathrm{PPh}_{3}\right)_{2} \\
\text { (blue) }\end{array}$ & $\begin{array}{c}\mathrm{tr} \\
\mathrm{P}-1 \\
2\end{array}$ & $\begin{array}{c}9.740 \\
9.797 \\
19.610\end{array}$ & $\begin{array}{c}99.9 \\
96.9 \\
105.6\end{array}$ & $\mathrm{PtC}_{2} \mathrm{P}_{2}$ & & & & & [10] \\
\hline $\begin{array}{c}\mathrm{Pt}\left\{\eta^{2}-\mathrm{CH}_{2} \mathrm{C}_{6} \mathrm{H}_{4} \mathrm{PBu}^{t}(\text { o-tolyl })\right\}_{2} \\
\text { (colourless) }\end{array}$ & $\begin{array}{c}\mathrm{m} \\
\mathrm{P} 2_{1} / \mathrm{n} \\
2\end{array}$ & $\begin{array}{l}12.59(2) \\
10.69(2) \\
12.44(2)\end{array}$ & $103.7(1)$ & $\mathrm{PtC}_{2} \mathrm{P}_{2}$ & $\begin{array}{l}\eta^{2} \mathrm{C} \\
\eta^{2} \mathrm{P}\end{array}$ & $\begin{array}{l}2.15(2) \\
2.238(4)\end{array}$ & $\mathrm{C}, \mathrm{P}$ & $82.6(6)^{c}$ & [11] \\
\hline $\begin{array}{l}\mathrm{Pt}\left(\mathrm{SMe}_{2}\right)_{2}(\mathrm{Ph}) \mathrm{Cl} \\
\quad \text { (pale yellow) }\end{array}$ & $\begin{array}{c}\mathrm{tr} \\
\mathrm{P}-1 \\
2\end{array}$ & $\begin{array}{l}7.9076(6) \\
8.994(6) \\
9.998(1)\end{array}$ & $\begin{array}{l}85.88(1) \\
80.600(9) \\
76.668(7)\end{array}$ & $\mathrm{PtS}_{2} \mathrm{CCl}$ & $\begin{array}{c}\mathrm{S} \\
\mathrm{PhC} \\
\mathrm{Cl}\end{array}$ & $\begin{array}{c}2.293(2,4) \\
2.004(5) \\
2.420(1)\end{array}$ & $\begin{array}{l}\mathrm{S}, \mathrm{S} \\
\mathrm{C}, \mathrm{Cl} \\
\mathrm{S}, \mathrm{C} \\
\mathrm{S}, \mathrm{Cl}\end{array}$ & $\begin{array}{c}178.51(5) \\
179.9(1) \\
89.5(1,4.5) \\
90.5(1,4.6)\end{array}$ & [12] \\
\hline $\begin{array}{l}\mathrm{Pt}\left(\mathrm{SMe}_{2}\right)_{2}(\mathrm{Ph}) \mathrm{Cl}^{\mathrm{d}} \\
\quad \text { (pale yellow) }\end{array}$ & $\begin{array}{c}\mathrm{m} \\
\mathrm{P} 2{ }_{1} / \mathrm{n} \\
8\end{array}$ & $\begin{array}{l}10.106(1) \\
13.046(3) \\
20.939(3)\end{array}$ & $94.39(1)$ & $\mathrm{PtS}_{2} \mathrm{CCl}$ & $\begin{array}{c}\mathrm{S} \\
\mathrm{PhC} \\
\mathrm{C}\end{array}$ & $\begin{array}{c}2.295(4,4) \\
1.99(1) \\
2.404(3)\end{array}$ & $\begin{array}{l}\mathrm{S}, \mathrm{S} \\
\mathrm{C}, \mathrm{Cl} \\
\mathrm{S}, \mathrm{C} \\
\mathrm{S}, \mathrm{C}\end{array}$ & $\begin{array}{l}170.8(1) \\
177.7(4) \\
85.7(4,3) \\
94.4(1,6)\end{array}$ & [12] \\
\hline & & & & $\mathrm{PtS}_{2} \mathrm{CCl}$ & $\begin{array}{c}\mathrm{S} \\
\mathrm{PhC} \\
\mathrm{C}\end{array}$ & $\begin{array}{c}2.287(4,8) \\
1.99(1) \\
2.403(4)\end{array}$ & $\begin{array}{l}\mathrm{S}, \mathrm{S} \\
\mathrm{C}, \mathrm{Cl} \\
\mathrm{S}, \mathrm{C} \\
\mathrm{S}, \mathrm{C}\end{array}$ & $\begin{array}{c}171.9(1) \\
177.2(3) \\
86.0(4,2) \\
94.1(1,1.1)\end{array}$ & \\
\hline $\begin{array}{c}\mathrm{Pt}\left(\mathrm{Pcy}_{3}\right)_{2}(\mathrm{H})\{\eta-\mathrm{C}(\mathrm{CHO})(\mathrm{CO})\} \\
(\text { pale yellow })\end{array}$ & $\begin{array}{c}\mathrm{m} \\
\mathrm{P} 2 / \mathrm{a} \\
4\end{array}$ & $\begin{array}{l}19.404(9) \\
11.889(6) \\
18.534(9)\end{array}$ & $91.3(1)$ & $\mathrm{PtP}_{2} \mathrm{HC}$ & $\begin{array}{c}\mathrm{P} \\
\mathrm{H} \\
\eta \mathrm{C}\end{array}$ & $\begin{array}{c}2.284(3,3) \\
\text { not given } \\
2.14(1)\end{array}$ & $\mathrm{P}, \mathrm{P}$ & $165.4(2)$ & [13] \\
\hline
\end{tabular}




\begin{tabular}{|c|c|c|c|c|c|c|c|c|c|}
\hline $\begin{array}{c}\mathrm{Pt}\left(\mathrm{PMe}_{3}\right)_{2}(\eta-\mathrm{hfp})(\mathrm{Me}) \\
(\text { white })\end{array}$ & $\begin{array}{c}\mathrm{m} \\
\mathrm{P} 2 / \mathrm{a} \\
4\end{array}$ & $\begin{array}{l}12.056(3) \\
16.938(3) \\
9.156(3)\end{array}$ & 104.91(2) & $\mathrm{PtP}_{2} \mathrm{OC}$ & $\begin{array}{c}\mathrm{Me}_{3} \mathrm{P} \\
\mathrm{MeC} \\
\eta \mathrm{O}\end{array}$ & $\begin{array}{c}2.181(4) \\
2.317(4) \\
2.13(2) \\
2.07(1)\end{array}$ & $\begin{array}{l}\mathrm{P}, \mathrm{P} \\
\mathrm{O}, \mathrm{C} \\
\mathrm{P}, \mathrm{O} \\
\mathrm{P}, \mathrm{C}\end{array}$ & $\begin{array}{c}101.6(2) \\
89.6(5) \\
80.6(3) \\
171.6(3) \\
88.3(5) \\
169.7(5)\end{array}$ & {$[15]$} \\
\hline $\begin{array}{c}{\left[\mathrm{Pt}\left(\mathrm{PMe}_{3}\right)_{2}(\eta-\mathrm{hfp})(\mathrm{Me})\right] \cdot \mathrm{hfpH}} \\
(\text { white })\end{array}$ & $\begin{array}{c}\operatorname{trg} \\
\mathrm{P} 3_{1} \\
3\end{array}$ & $\begin{array}{l}9.347(6) \\
23.317(3)\end{array}$ & & $\mathrm{PtP}_{2} \mathrm{OC}$ & $\begin{array}{c}\mathrm{Me}_{3} \mathrm{P} \\
\mathrm{MeC} \\
\eta \mathrm{O}\end{array}$ & $\begin{array}{c}2.199(8) \\
2.334(8) \\
2.07(3) \\
2.06(3)\end{array}$ & $\begin{array}{l}\mathrm{P}, \mathrm{P} \\
\mathrm{O}, \mathrm{C} \\
\mathrm{P}, \mathrm{O} \\
\mathrm{P}, \mathrm{C}\end{array}$ & $\begin{array}{c}96.9(3) \\
86(1) \\
88.0(7) \\
175.0(7) \\
89(1), 174(1)\end{array}$ & {$[16]$} \\
\hline $\begin{array}{c}\operatorname{Pt}\left(\eta^{3} \text {-bdpep }\right) \mathrm{Cl} \\
\quad \text { (white })\end{array}$ & $\begin{array}{c}\text { or } \\
\text { Pbca } \\
8\end{array}$ & $\begin{array}{l}21.807(3) \\
17.020(5) \\
16.519(2)\end{array}$ & & $\mathrm{PtP}_{2} \mathrm{CCl}$ & $\begin{array}{c}\eta^{3} \mathrm{P} \\
\eta^{3} \mathrm{C} \\
\mathrm{Cl}\end{array}$ & $\begin{array}{l}2.261(6,8) \\
2.200(2) \\
2.368(6)\end{array}$ & $\begin{array}{c}\mathrm{P}, \mathrm{P} \\
\mathrm{C}, \mathrm{Cl} \\
\mathrm{P}, \mathrm{C} \\
\mathrm{P}, \mathrm{Cl}\end{array}$ & $\begin{array}{c}163.4(2) \\
178.5(5) \\
81.7(6,5)^{\mathrm{c}} \\
98.3(2,8)\end{array}$ & [17] \\
\hline $\begin{array}{c}{\left[\mathrm{Pt}\left(\eta^{3} \text {-bdpep }\right) \mathrm{Cl}\right] . \mathrm{CHCl}_{3}} \\
\text { (white) }\end{array}$ & $\begin{array}{c}\mathrm{m} \\
\mathrm{P} 2_{1} / \mathrm{c} \\
4\end{array}$ & $\begin{array}{l}11.850(2) \\
15.150(3) \\
19.837(2)\end{array}$ & $98.69(1)$ & $\mathrm{PtP}_{2} \mathrm{CCl}$ & $\begin{array}{c}\eta^{3} \mathrm{P} \\
\eta^{3} \mathrm{C} \\
\mathrm{Cl}\end{array}$ & $\begin{array}{l}2.271(2,3) \\
2.003(9) \\
2.384(2)\end{array}$ & $\begin{array}{c}\mathrm{P}, \mathrm{P} \\
\mathrm{C}, \mathrm{Cl} \\
\mathrm{P}, \mathrm{C} \\
\mathrm{P}, \mathrm{Cl}\end{array}$ & $\begin{array}{c}163.1(1) \\
177.6(3) \\
81.8(3,6)^{\mathrm{c}} \\
98.3(1,1)\end{array}$ & [17] \\
\hline $\begin{array}{c}{\left[\mathrm{Pt}\left\{\eta^{3}-\mathrm{CH}_{2} \mathrm{C}(\mathrm{SPh}) \mathrm{CH}_{2}\right\} .\right.} \\
\left.\left(\mathrm{PPh}_{3}\right)_{2}\right] \mathrm{BF}_{4} \mathrm{CHCl}_{3} \\
\text { (pale yellow) }\end{array}$ & $\begin{array}{c}\operatorname{tr} \\
\mathrm{P}-1 \\
2\end{array}$ & $\begin{array}{l}12.842(3) \\
12.989(3) \\
13.589(5)\end{array}$ & $\begin{array}{l}89.85(2) \\
92.98(3) \\
106.66(2)\end{array}$ & $\mathrm{PtC}_{3} \mathrm{P}_{2}$ & $\begin{array}{l}\eta^{3} \mathrm{C} \\
\mathrm{Ph}_{3} \mathrm{P}\end{array}$ & $\begin{array}{l}2.177(6,0) \\
2.204(6) \\
2.290(2,4)\end{array}$ & $\begin{array}{l}\mathrm{C}, \mathrm{C} \\
\mathrm{P}, \mathrm{P} \\
\mathrm{C}, \mathrm{P}\end{array}$ & $\begin{array}{c}37.1(2,5)^{\mathrm{e}} \\
66.9(2) \\
101.90(6) \\
95.3(2,2.8) \\
128.6(2,1.9) \\
161.3(2,2.9)\end{array}$ & [19] \\
\hline $\begin{array}{c}{\left[\mathrm{Pt}\left\{\eta^{3}-\mathrm{CH}_{2} \mathrm{C}(\mathrm{SPh}) \mathrm{CH}_{2}\right\} .\right.} \\
\left.\left(\mathrm{PPh}_{3}\right)_{2}\right] \mathrm{BF}_{4} \mathrm{CHCl}_{3} \\
\text { (pale yellow) }\end{array}$ & $\begin{array}{c}\operatorname{tr} \\
\mathrm{P}-1 \\
2\end{array}$ & $\begin{array}{l}11.285(3) \\
11.615(2) \\
17.946(2)\end{array}$ & $\begin{array}{l}106.57(1) \\
91.44(1) \\
106.43(2)\end{array}$ & $\mathrm{PtC}_{3} \mathrm{P}_{2}$ & & & & & {$[20]$} \\
\hline $\begin{array}{c}\left(\mathrm{PPh}_{4}\right)\left[\mathrm{PtCl}_{3}\left(\eta^{2}-\mathrm{C}_{4} \mathrm{H}_{8} \mathrm{O}_{2}\right)\right] \\
\text { (yellow) }\end{array}$ & $\begin{array}{c}\mathrm{m} \\
\mathrm{P} 2_{1} / \mathrm{c} \\
4\end{array}$ & $\begin{array}{c}13.665 \\
7.471 \\
27.402\end{array}$ & 102.08 & $\mathrm{PtCl}_{3} \mathrm{C}_{2}$ & $\begin{array}{c}\mathrm{Cl} \\
\eta^{2} \mathrm{C}\end{array}$ & $\begin{array}{c}2.296(9,5) \\
2.14(3,4)\end{array}$ & $\begin{array}{l}\mathrm{Cl}, \mathrm{Cl} \\
\mathrm{C}, \mathrm{C}\end{array}$ & $\begin{array}{l}88.8(3,4) \\
177.3(4) \\
38.1(9)^{\mathrm{e}}\end{array}$ & {$[21]$} \\
\hline $\begin{array}{c}\left(\mathrm{NEt}_{4}\right)\left[\mathrm{PtCl}_{3}\left(\eta^{2}-\mathrm{C}_{4} \mathrm{H}_{8} \mathrm{O}_{2}\right)\right] \\
\text { (pale yellow) }\end{array}$ & $\begin{array}{c}\mathrm{m} \\
\mathrm{P} 2_{1} / \mathrm{n} \\
4\end{array}$ & $\begin{array}{c}7.728(4) \\
17.763(6) \\
13.418(9)\end{array}$ & 103.71(1) & $\mathrm{PtCl}_{3} \mathrm{C}_{2}$ & $\begin{array}{l}\mathrm{Cl} \\
\eta^{2} \mathrm{C}\end{array}$ & $\begin{array}{c}2.300(2,8) \\
2.324(2) \\
2.128(7) \\
2.208(7)\end{array}$ & $\begin{array}{l}\mathrm{Cl}, \mathrm{Cl} \\
\mathrm{C}, \mathrm{C} \\
\mathrm{Cl}, \mathrm{C}\end{array}$ & $\begin{array}{c}90.0(1,8) \\
179.2(9) \\
37.1(3)^{\mathrm{e}} \\
161.5(3,4.3)\end{array}$ & {$[22]$} \\
\hline
\end{tabular}


(Table 1). Contd.....

\begin{tabular}{|c|c|c|c|c|c|c|c|c|c|}
\hline $\begin{array}{c}\mathrm{PtCl}_{3}\left(\eta^{2}-\mathrm{C}_{4} \mathrm{H}_{10} \mathrm{~N}-2\right) \\
\text { (orange yellow) }\end{array}$ & $\begin{array}{c}\mathrm{m} \\
\mathrm{P} 2{ }_{1} / \mathrm{c} \\
4\end{array}$ & $\begin{array}{c}10.248(3) \\
10.388(1) \\
8.414(1)\end{array}$ & $97.96(2)$ & $\mathrm{PtCl}_{3} \mathrm{C}_{2}$ & $\begin{array}{c}\mathrm{Cl} \\
\eta^{2} \mathrm{C}\end{array}$ & $\begin{array}{c}2.298(7,10) \\
2.339(7) \\
2.14(3,2)\end{array}$ & $\begin{array}{l}\mathrm{Cl}, \mathrm{Cl} \\
\mathrm{C}, \mathrm{C}\end{array}$ & $\begin{array}{l}89.0(3,6) \\
177.2(2) \\
38.1(10)^{\mathrm{e}}\end{array}$ & [23] \\
\hline $\begin{array}{c}\mathrm{PtCl}_{3}\left(\eta^{2}-\mathrm{C}_{4} \mathrm{H}_{10} \mathrm{~N}-2\right) \\
(\text { yellow })\end{array}$ & $\begin{array}{c}\mathrm{m} \\
\mathrm{P} 2_{1} / \mathrm{c} \\
4\end{array}$ & $\begin{array}{c}7.2254(4) \\
7.521(1) \\
16.596(1)\end{array}$ & $92.77(2)$ & $\mathrm{PtCl}_{3} \mathrm{C}_{2}$ & $\begin{array}{c}\mathrm{Cl} \\
\eta^{2} \mathrm{C}\end{array}$ & $\begin{array}{c}2.297(6,4) \\
2.320(6) \\
2.165(20,15)\end{array}$ & $\begin{array}{l}\mathrm{Cl}, \mathrm{Cl} \\
\mathrm{C}, \mathrm{C}\end{array}$ & $\begin{array}{l}88.8(3,2) \\
177.5(2) \\
37.8(10)^{\mathrm{e}}\end{array}$ & [24] \\
\hline $\begin{array}{c}\mathrm{PtCl}_{3}\left(\eta^{2}-\mathrm{C}_{5} \mathrm{H}_{12} \mathrm{~N}-4\right) \\
\text { (yellow) }\end{array}$ & $\begin{array}{c}\mathrm{m} \\
\mathrm{P} 2{ }_{1} / \mathrm{c} \\
4\end{array}$ & $\begin{array}{c}6.435(1) \\
9.372(11) \\
16.737(13)\end{array}$ & $100.6(1)$ & $\mathrm{PtCl}_{3} \mathrm{C}_{2}$ & $\begin{array}{c}\mathrm{Cl} \\
\eta^{2} \mathrm{C}\end{array}$ & $\begin{array}{c}2.321(9,0) \\
2.335(9) \\
2.182(30,12)\end{array}$ & $\begin{array}{l}\mathrm{Cl}, \mathrm{Cl} \\
\mathrm{C}, \mathrm{C}\end{array}$ & $\begin{array}{c}89.2(3,1.3) \\
177.5(3) \\
37.6(10)^{\mathrm{e}}\end{array}$ & [25] \\
\hline $\begin{array}{c}\mathrm{PtCl}_{3}\left(\eta^{2}-\mathrm{C}_{5} \mathrm{H}_{12} \mathrm{~N}-4\right) \\
\text { (orange) }\end{array}$ & $\begin{array}{c}\mathrm{m} \\
\mathrm{P} 2 / \mathrm{c} \\
4\end{array}$ & $\begin{array}{c}10.504(7) \\
10.627(10) \\
9.608(8)\end{array}$ & $106.9(1)$ & $\mathrm{PtCl}_{3} \mathrm{C}_{2}$ & $\begin{array}{c}\mathrm{Cl} \\
\eta^{2} \mathrm{C}\end{array}$ & $\begin{array}{c}2.301(6,13) \\
2.342(6) \\
2.129(23,19)\end{array}$ & $\begin{array}{l}\mathrm{Cl}, \mathrm{Cl} \\
\mathrm{C}, \mathrm{C}\end{array}$ & $\begin{array}{c}89.7(2,9) \\
175.2(2) \\
38.8(8)^{\mathrm{e}}\end{array}$ & [25] \\
\hline $\begin{array}{c}\mathrm{PtCl}_{3}\left(\eta^{2}-\mathrm{C}_{5} \mathrm{H}_{12} \mathrm{~N}-2\right) \\
(\text { yellow })\end{array}$ & $\begin{array}{c}\mathrm{m} \\
\mathrm{P} 2{ }_{1} / \mathrm{c} \\
4\end{array}$ & $\begin{array}{c}11.215(3) \\
8.729(2) \\
10.259(3)\end{array}$ & $90.01(1)$ & $\mathrm{PtCl}_{3} \mathrm{C}_{2}$ & $\begin{array}{c}\mathrm{Cl} \\
\eta^{2} \mathrm{C}\end{array}$ & $\begin{array}{c}2.314(5,2) \\
2.321(4) \\
2.097(14) \\
2.183(14)\end{array}$ & $\begin{array}{l}\mathrm{Cl}, \mathrm{Cl} \\
\mathrm{C}, \mathrm{C}\end{array}$ & $\begin{array}{c}88.4(1,4) \\
174.5(1) \\
38.1(5)^{\mathrm{e}}\end{array}$ & [26] \\
\hline $\begin{array}{c}\mathrm{PtCl}_{3}\left(\eta^{2}-\mathrm{C}_{5} \mathrm{H}_{12} \mathrm{~N}-2\right) \\
(\text { yellow })\end{array}$ & $\begin{array}{c}\mathrm{m} \\
\mathrm{P} 2 / \mathrm{c} \\
4\end{array}$ & $\begin{array}{c}10.530(3) \\
9.037(3) \\
10.067(3)\end{array}$ & $105.50(3)$ & $\mathrm{PtCl}_{3} \mathrm{C}_{2}$ & $\begin{array}{c}\mathrm{Cl} \\
\eta^{2} \mathrm{C}\end{array}$ & $\begin{array}{c}2.306(3,1) \\
2.330(7) \\
2.156(13,10)\end{array}$ & $\begin{array}{l}\mathrm{Cl}, \mathrm{Cl} \\
\mathrm{C}, \mathrm{C}\end{array}$ & $\begin{array}{c}89.1(1,1.0) \\
174.3(1) \\
37.9(4)^{\mathrm{e}}\end{array}$ & [27] \\
\hline $\begin{array}{c}{\left[\mathrm{PtCl}_{3}\left(\eta^{2}-\mathrm{C}_{4} \mathrm{H}_{12} \mathrm{~N}_{2}\right)\right] \mathrm{Cl}} \\
(\text { yellow })\end{array}$ & $\begin{array}{c}\mathrm{m} \\
\mathrm{P} 2 / \mathrm{c} \\
4\end{array}$ & $\begin{array}{c}11.238(3) \\
13.234(4) \\
7.769(1)\end{array}$ & $112.99(2)$ & $\mathrm{PtCl}_{3} \mathrm{C}_{2}$ & $\begin{array}{c}\mathrm{Cl} \\
\eta^{2} \mathrm{C}\end{array}$ & $\begin{array}{c}2.305(4,4) \\
2.330(4) \\
2.134(15,2)\end{array}$ & $\begin{array}{l}\mathrm{Cl}, \mathrm{Cl} \\
\mathrm{C}, \mathrm{C}\end{array}$ & $\begin{array}{c}89.0(2,5) \\
177.7(2) \\
39.5(6)^{\mathrm{e}}\end{array}$ & [27] \\
\hline $\begin{array}{c}{\left[\mathrm{PtCl}_{3}\left(\eta^{2}-\mathrm{C}_{4} \mathrm{H}_{12} \mathrm{~N}_{2}\right)\right] \mathrm{Cl} .0 .5 \mathrm{H}_{2} \mathrm{O}} \\
(\text { yellow })\end{array}$ & $\begin{array}{c}\mathrm{m} \\
\mathrm{C} 2 / \mathrm{c} \\
4\end{array}$ & $\begin{array}{c}23.911(7) \\
6.942(1) \\
13.956(2)\end{array}$ & $102.07(2)$ & $\mathrm{PtCl}_{3} \mathrm{C}_{2}$ & $\begin{array}{c}\mathrm{Cl} \\
\eta^{2} \mathrm{C}\end{array}$ & $\begin{array}{c}2.302(2,9) \\
2.342(2) \\
2.130(8,19)\end{array}$ & $\begin{array}{l}\mathrm{Cl} . \mathrm{Cl} \\
\mathrm{C}, \mathrm{C}\end{array}$ & $\begin{array}{c}88.6(1,5) \\
176.11(9) \\
38.9(3)^{\mathrm{e}}\end{array}$ & [28] \\
\hline $\begin{array}{c}\mathrm{PtCl}_{3}\left(\eta^{2}-\mathrm{C}_{5} \mathrm{H}_{11} \mathrm{NMe}\right) \\
(\text { yellow })\end{array}$ & $\begin{array}{c}\text { or } \\
\text { Pna } 2_{1} \\
4\end{array}$ & $\begin{array}{l}18.623(7) \\
8.385(2) \\
7.261(2)\end{array}$ & & $\mathrm{PtCl}_{3} \mathrm{C}_{2}$ & $\begin{array}{c}\mathrm{Cl} \\
\eta^{2} \mathrm{C}\end{array}$ & $\begin{array}{c}2.273(9,12) \\
2.336(2) \\
2.19(4) \\
2.24(3)\end{array}$ & $\mathrm{C}, \mathrm{C}$ & $\begin{array}{c}91.6(3,5) \\
176.7(3) \\
37(1)^{\mathrm{e}}\end{array}$ & [29] \\
\hline $\begin{array}{c}{\left[\mathrm{Pt}\left(\mathrm{Cl}_{3}\right)\left(\eta^{3}-\mathrm{C}_{5} \mathrm{H}_{11} \mathrm{NMe}\right)\right] \cdot \mathrm{H}_{2} \mathrm{O}} \\
(\text { yellow })\end{array}$ & $\begin{array}{c}\mathrm{m} \\
\mathrm{P} 2{ }_{1} / \mathrm{c} \\
4\end{array}$ & $\begin{array}{c}11.500(4) \\
6.088(4) \\
18.961(5)\end{array}$ & $116.92(4)$ & $\mathrm{PtCl}_{3} \mathrm{C}_{2}$ & $\begin{array}{c}\mathrm{Cl} \\
\eta^{2} \mathrm{C}\end{array}$ & $\begin{array}{c}2.289(4) \\
2.329(4,5) \\
2.141(12,10)\end{array}$ & $\mathrm{C}, \mathrm{C}$ & $\begin{array}{c}88.8(1,6) \\
174.8(2) \\
38.2(6)^{\mathrm{e}}\end{array}$ & [30] \\
\hline $\begin{array}{c}\operatorname{Pt}\left(\eta^{2}-\mathrm{MeCH}=\mathrm{CHMe}\right) \mathrm{Cl}_{2} \\
\left(\eta^{1}-\text { pea }\right) \\
(\text { yellow })\end{array}$ & $\begin{array}{c}\mathrm{m} \\
\mathrm{P} 2_{1} \\
2\end{array}$ & $\begin{array}{l}12.87(2) \\
6.58(1) \\
8.86(1)\end{array}$ & $102.38(15)$ & $\mathrm{PtC}_{2} \mathrm{Cl}_{2} \mathrm{~N}$ & $\begin{array}{c}\eta^{2} \mathrm{C} \\
\mathrm{Cl} \\
\eta^{1} \mathrm{~N}\end{array}$ & $\begin{array}{c}2.16(5,1) \\
2.32(2,3) \\
2.14(3)\end{array}$ & $\begin{array}{l}\mathrm{Cl}, \mathrm{Cl} \\
\mathrm{Cl}, \mathrm{N}\end{array}$ & $\begin{array}{l}89(1) \\
90(1) \\
176(1)\end{array}$ & [31] \\
\hline $\begin{array}{c}\operatorname{Pt}\left(\eta^{2}-\mathrm{MeCH}=\mathrm{CHMe}\right) \mathrm{Cl}_{2} . \\
\left(\eta^{1} \text {-pea }\right) \\
(\text { yellow })\end{array}$ & $\begin{array}{c}\text { or } \\
\mathrm{P} 2_{1} 2_{1} 2_{1} \\
4\end{array}$ & $\begin{array}{l}15.64(5) \\
10.42(3) \\
9.16(3)\end{array}$ & & $\mathrm{PtC}_{2} \mathrm{Cl}_{2} \mathrm{~N}$ & $\begin{array}{c}\eta^{2} \mathrm{C} \\
\mathrm{Cl} \\
\eta^{1} \mathrm{~N}\end{array}$ & $\begin{array}{l}\text { not given } \\
2.34(2,0) \\
\text { not given }\end{array}$ & $\mathrm{Cl}, \mathrm{Cl}$ & 180 & [32] \\
\hline $\begin{array}{l}\operatorname{Pt}\left(\eta^{4}-\mathrm{dph}\right) \mathrm{Cl}_{2} \\
\text { (pale yellow) }\end{array}$ & $\begin{array}{c}\mathrm{m} \\
\mathrm{C} 2 / \mathrm{c} \\
4\end{array}$ & $\begin{array}{l}18.258(5) \\
8.395(3) \\
11.043(2)\end{array}$ & $108.5(6)$ & $\mathrm{PtC}_{4} \mathrm{Cl}_{2}$ & $\eta^{4} \mathrm{C}$ & $\begin{array}{l}2.19(2,0) \\
2.30(2,0) \\
2.324(5,0)\end{array}$ & $\begin{array}{c}\mathrm{C}, \mathrm{C} \\
\mathrm{Cl}, \mathrm{Cl} \\
\mathrm{C}, \mathrm{Cl}\end{array}$ & $\begin{array}{c}37.5(8)^{\mathrm{e}} \\
81.9(7,3.3) \\
11.0(7) \\
83.9(5) \\
101.0(4) \\
161.8(7)\end{array}$ & [33] \\
\hline
\end{tabular}


(Table 1). Contd.....

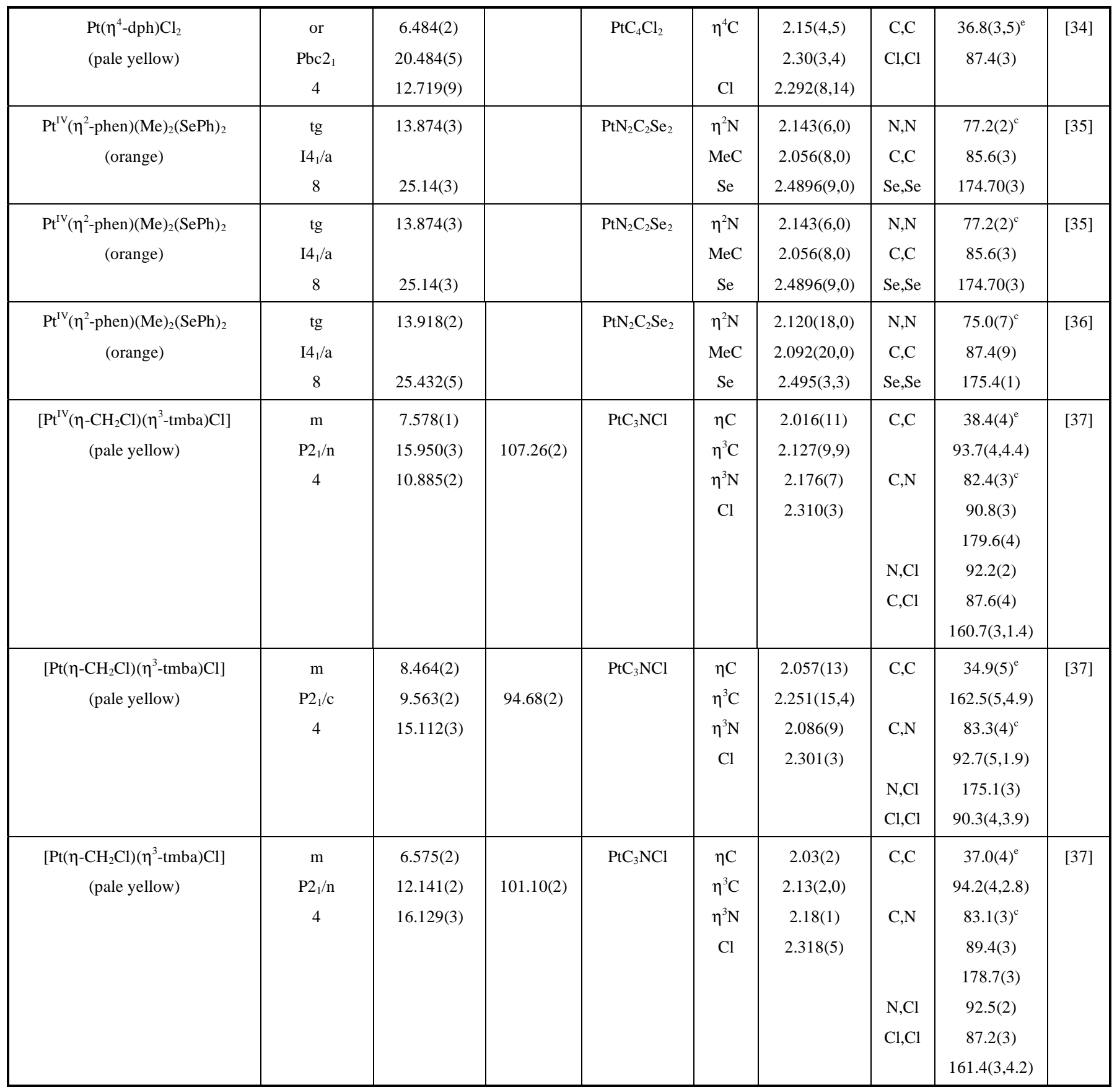

Footnotes: a.) Where more than one chemically equivalent distance or angle is present, the mean value is tabulated. The first number in parenthesis is the e.s.d., and the second is the maximum deviation from the mean.

b). The chemical identity of the coordinated atom or ligand is specified in these columns

c). Five-membered metallocyclic ring.

d). There are two crystallographically independent molecules.

e). Three membered metallocyclic ring.

one is monoclinic and the other triclinic [10,12]. For the remainder, the mixture is monoclinic and trigonal $[15,16]$.

There is an example, $\left[\mathrm{Pt}\left(\mathrm{SMe}_{2}\right)_{2}(\mathrm{Ph})(\mathrm{Cl})\right]$ [12] which exists in two isomeric forms where one form contains two crystallographically independent molecules.

Each $\mathrm{Pt}(\mathrm{II})$ atom with the chromophores: $\mathrm{PtC}_{4}, \mathrm{PtC}_{2} \mathrm{P}_{2}$, $\mathrm{PtS}_{2} \mathrm{CCl}, \mathrm{PtP}_{2} \mathrm{HC}, \mathrm{PtP}_{2} \mathrm{OC}$ and $\mathrm{PtP}_{2} \mathrm{CCl}$ has a square-planar arrangement with a different degree of distortion [9-17]. The remaining $\mathrm{Pt}(\mathrm{II})$ examples with the chromophores: $\mathrm{PtC}_{3} \mathrm{X}_{2}$ $(\mathrm{X}=\mathrm{Cl}[8]$ or $\mathrm{P}[19,20]), \mathrm{PtCl}_{3} \mathrm{C}_{2}[21-30]$ and $\mathrm{PtC}_{2} \mathrm{Cl}_{2} \mathrm{~N}$
$[31,32]$ and $\mathrm{PtC}_{4} \mathrm{Cl}_{2}[33,34]$ are a $\pi$ - complexes. Each $\mathrm{Pt}(\mathrm{II})$ atom $\left(\mathrm{PtC}_{3} \mathrm{X}_{2}\right)$ is found in the square-planar arrangement with two $\mathrm{X}$ in cis - position, The one $\mathrm{C}$ atom $\sigma$ bonded to the $\mathrm{Pt}$ atom ( $\mathrm{Pt}-\mathrm{C}, 2.40 \AA$ average) and the center of the doubled bond $(\mathrm{Pt}-\mathrm{C}, \approx 2.10 \AA$ of the respective tridentate $\mathrm{C}$ donor atoms ligand, occupy the other sites of coordination. Each $\mathrm{Pt}(\mathrm{II})$ atom in $\mathrm{PtCl}_{3} \mathrm{C}_{2}[21-30]$ is square coordinated with three chlorine atoms and the double bond of the respective ligand. In $\mathrm{Pt}\left(\eta^{4}-\mathrm{dph}\right) \mathrm{Cl}_{2}$ monoclinic [33] and orthorhombic [34] a square planar geometry about each $\mathrm{Pt}(\mathrm{II})$ atom is built up by two chlorine atoms in cis- position 
and two centers of double bond of the 2,5 - diphenyl-1,5hexadiene ligand.

Each $\mathrm{Pt}(\mathrm{IV})$ atom in $\mathrm{Pt}\left(\eta^{2}\right.$-phen $)(\mathrm{Me})_{2}(\mathrm{SePh})_{2}[35,36]$ is a tetragonal -.bipyramidally coordinated $\left(\mathrm{PtN}_{2} \mathrm{C}_{2} \mathrm{Se}_{2}\right)$ with $\mathrm{Se}$ atom in the axial positions with $\mathrm{Se}-\mathrm{Pt}-\mathrm{Se}$ bond angles of $174.6^{\circ}$ (average).

As mentioned above, pale yellow $\left[\mathrm{Pt}\left(\eta-\mathrm{CH}_{2} \mathrm{Cl}\right)\left(\eta^{3}-\right.\right.$ tmba)Cl] [37] exists in three monoclinic isomeric form and one example structure is shown in (Fig. 1). In all three molecules the five membered ring $\mathrm{Pt}, \mathrm{N}, \mathrm{C}(1), \mathrm{C}(2), \mathrm{C}(3)$ has a $\mathrm{C}(2)$ envelope conformation, with the $\mathrm{Pt}, \mathrm{C}(3), \mathrm{C}(4)$ plane inclined at $101.4,98.9$ and $102.9^{\circ}$, respectively to the coordination plane Pt, N, C(1), C(9). Otherwise, the bond lengths and angles are not unusual (Table $\mathbf{1}$ ).

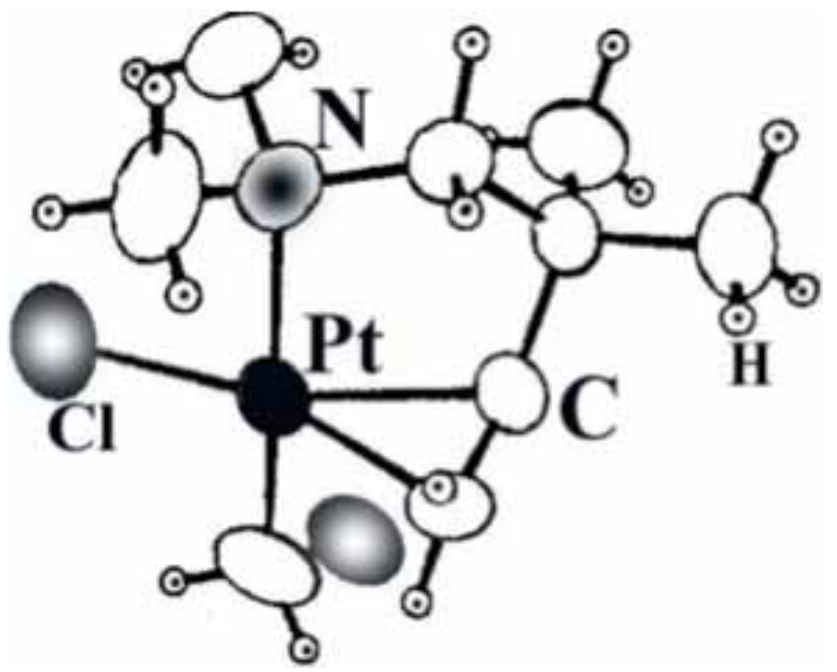

Fig. (1). Structure of one isomeric form of $\left[\mathrm{Pt}^{\mathrm{II}}\left(\eta-\mathrm{CH}_{2} \mathrm{Cl}\right)\left(\eta^{3}-\right.\right.$ tmba)Cl] [37].

\subsubsection{Crystallographically Independent Molecules}

Crystallographic and structural parameters of monomeric platinum organometllic compounds contain two, three and four crystallographically independent molecules within the same crystal are given in Table 2 . There are thirty six derivatives, which contain two crystallographically independent molecules within the same crystal [12, 37-71]. One derivative contains three such molecules [72] and another one derivatives has four such molecules [73]. In these derivatives the platinum atoms are found in the oxidation states of zero (x5) [38-42], +2 (x 27) [12, 37, 43-65, 72, 73] and +4 (x 6) [66-71]. The derivatives belong to the triclinic (x 17), monoclinic (x 16) and orthorhombic (x 5) classes.

In pale yellow $\operatorname{Pt}\left(\eta^{2}-\text { bchp }\right)_{2}$ [38] the pair of homobidentate bchp ligands ( $\mathrm{C}, \mathrm{C}^{\prime}$-donors) with two three - membered rings creating a distorted tetrahedral geometry about the $\mathrm{Pt}(\mathrm{O})$ atom $\left(\mathrm{PtC}_{4}\right)$ with differing degrees of distortion. The mean $\mathrm{Pt}-\mathrm{C}$ bond distances are $1.99 \AA$ (molecule 1) and $2.01 \AA$ (molecule 2).

In another three $\operatorname{Pt}(0)$ derivatives $\operatorname{Pt}\left(\eta^{2}-\mathrm{CL}\right)\left(\mathrm{PPh}_{3}\right)_{2}(\mathrm{CL}$ is $\mathrm{HC}=\mathrm{C}_{6} \mathrm{H}_{10} \mathrm{OH}$ [39], had [40] and ben [41] a homobidentate $\left(\mathrm{C}, \mathrm{C}^{\prime}\right.$ - donors ) ligand and two $\mathrm{PPh}_{3}$ ligands create a tetrahedral arrangement about each $\mathrm{Pt}(0)$ atom $\left(\mathrm{PtC}_{2} \mathrm{P}_{2}\right)$, with differing degrees of distortion. In $\mathrm{Pt}^{0}\left(\eta^{2}\right.$-sti) $\left(\eta^{2}\right.$-chirophos $)$ [42] a homobidentate sti $\left(C, C^{\prime}\right.$ - donors and chirophos (P, $\mathrm{P}^{\prime}$ donors) ligands form two metallocycles, three - and five - membered, with the mean $\mathrm{C}-\mathrm{Pt}-\mathrm{C}$ and $\mathrm{P}-\mathrm{Pt}-\mathrm{P}$ bite angles of $39.3(2)^{\circ}$, and $87.13 .(6)^{\circ}$ (molecule 1), and $39.9(3)^{\circ}$ and $86.55(6)^{\circ}$ (molecule 2 ).

The overall data (Table 2) shows that there are twenty two Pt(II) derivatives in which each $\mathrm{Pt}(\mathrm{II})$ atom has a square planar geometry with different degrees of distortion. There is wide variety of the chromophores: $\mathrm{PtN}_{3} \mathrm{C}$ [43], $\mathrm{PtN}_{2} \mathrm{C}_{2}$ [44], $\mathrm{PtC}_{2} \mathrm{P}_{2}$ [45-47], $\mathrm{PtN}_{2} \mathrm{CX}(\mathrm{X}=\mathrm{Cl}[48,49]$ or $\mathrm{P}[50]), \mathrm{PtC}_{2} \mathrm{NX}$ $\left(\mathrm{X}=\mathrm{Cl}\right.$ [51] or I [52]), $\mathrm{PtCl}_{2} \mathrm{NC}[53,54], \mathrm{PtS}_{2} \mathrm{CCl}$ [12], $\mathrm{PtP}_{2} \mathrm{HC}[55], \mathrm{PtP}_{2} \mathrm{CX}(\mathrm{X}=\mathrm{Cl}[56,57]$ or I [58]), [PtNCClP [59, 60], PtCCISP [61] and PtNCSAs [73].

Remaining $\mathrm{Pt}(\mathrm{II})$ derivatives with the chromophores about the $\mathrm{Pt}(\mathrm{II})$ atoms: $\mathrm{PtC}_{3} \mathrm{X}_{2}(\mathrm{X}=\mathrm{Cl}$ [37] or $\mathrm{P}$ [62]), $\mathrm{PtC}_{2} \mathrm{Cl}_{2} \mathrm{~N}$ [63] , $\mathrm{PtC}_{4} \mathrm{NS}$ [64] and $\mathrm{PtC}_{3} \mathrm{ClP}$ [72] are $\pi$ complexes. Each $\mathrm{Pt}(\mathrm{II})$ atom is square coordinated.

The structure of dark red $\left[\mathrm{Pt}\left(\eta^{2}-\mathrm{PhC} \equiv \mathrm{CPh}\right)\left(\eta^{2}-\right.\right.$ dmphen) $\mathrm{I}_{2}$ ]. $\mathrm{CHCl}_{3}$ [65] contains two crystallographically independent molecules, of which one is shown in (Fig. 2), and disordered chloroform molecule. Each complex has a crystallographically imposed $\mathrm{C}_{\mathrm{s}}$ and an approximate $\mathrm{C}_{2 \mathrm{v}}$ symmetry with the mirror plane bisecting the dmphen and the diphenylacetylene moieties and comprising the PtI2 fragment The platinum(II)atom exhibits a tetragonal bipyramidal coordination. In the axial positions are iodides atoms and the equatorial sites are occupied by the two nitrogen atoms of the bidentate dmphen ligand and by carbon atoms of diphenylacetylene $\left(\mathrm{PtN}_{2} \mathrm{C}_{2} \mathrm{I}_{2}\right)$.

In six $\mathrm{Pt}(\mathrm{IV})$ derivatives $\left[\mathrm{Pt}\left(\eta^{2}-\mathrm{bpy}\right)\left(\eta^{2}-\mathrm{mq}\right)(\mathrm{Me})_{2}\right]_{2} \mathrm{BF}_{4}$ [66], $\left[\mathrm{Pt}\left(\eta^{2}-\mathrm{htpzb}\right)(\mathrm{Ph})_{2}(\mathrm{I})_{2}\right]_{2}(\mathrm{I})\left(\mathrm{I}_{3}\right) \quad[67], \quad \mathrm{Pt}\left(\mathrm{CH}_{2} \mathrm{Ph}\right)\left(\eta^{2}-\right.$ thpy $\left.)_{2} \mathrm{Br}\right]$ [68], [Pt(Me $\left.)_{3}\left(\eta^{2}-\mathrm{dmpbpy}\right) \mathrm{I}\right]$ [69], $\left[\operatorname{Pt}(\mathrm{py})_{2}\left\{\eta^{2}-\right.\right.$ $\left.\left.\mathrm{C}_{3} \mathrm{H}_{4}(\mathrm{Ph})_{2}\right\} \mathrm{Cl}_{2}\right]$ [70] and [Pt $\left.\left(\mathrm{Me}_{3}\right)\left(\eta^{2}-\mathrm{C}_{33} \mathrm{H}_{31} \mathrm{NP}_{2}\right) \mathrm{I}\right]$ [71] each $\mathrm{Pt}(\mathrm{IV})$ atoms is octahedrally coordinated : $\mathrm{PtN}_{3} \mathrm{C}_{3}$ [66], $\mathrm{PtN}_{3} \mathrm{C}_{2} \mathrm{I}$ [67], $\mathrm{PtC}_{3} \mathrm{~N}_{2} \mathrm{X}$ (X = Br [68] or I [69]), $\mathrm{PtN}_{2} \mathrm{C}_{2} \mathrm{Cl}_{2}$ [70] and $\mathrm{PtC}_{3} \mathrm{NPI}$ [71], with different degrees of distortion (Table 2).

\subsection{Bi -, Tri- and Tetranuclear Complexes}

There are eleven binuclear [74-83], three trinuclear [8486] and three tetranuclear [87-89] derivatives which contain two crystallographically independent molecules within the same crystal and their structural parameters are given in Table 3 .

Yellow $\mathrm{Pt}_{2}(\mu-\mathrm{S})(\mathrm{CO})_{2}\left(\mathrm{PPh}_{3}\right)_{2}$ [74] is only example with $\mathrm{Pt}(\mathrm{I})$. In each dimer, the $\left(\mathrm{PPh}_{3}\right)(\mathrm{CO}) \mathrm{Pt}-\mathrm{Pt}(\mathrm{CO})\left(\mathrm{PPh}_{3}\right)$ unit with $\mathrm{Pt}(\mathrm{I})-\mathrm{Pt}(\mathrm{I})$ bond distances of 2.5998(8) and 2.6008(8) $\AA$, is bridged by a single $\mu-\mathrm{S}$ atom. The $\mathrm{Pt}-\mathrm{S}-\mathrm{Pt}$ bridge angles are 70.1(1) and $70.2(1)^{\circ}$, respectively.

In pale orange derivative $\left[\mathrm{Pt}_{2}(\mu-\mathrm{C}=\mathrm{CHPh})\left(\mathrm{PEt}_{3}\right)_{3} \quad[75]\right.$ non-equivalent fragments, $\left(\mathrm{PEt}_{3}\right)(\mathrm{Br}) \mathrm{Pt}$ and $\mathrm{Pt}(\mathrm{Br})\left(\mathrm{PEt}_{3}\right)_{2}$, are both bridged unsymmetrically by a $\mu-\mathrm{C}=\mathrm{CHPh}$ ligand and linked by a Pt(II) - Pt(II) bond. The Pt $-\mathrm{Pt}$ bond distances are 2.682(1) and 2.687(1) $\AA$, respectively.

Structure of yellow derivative [76] consists of discrete $\left[\mathrm{Pt}_{2}(\mu-\mathrm{H})\left(\mu-\mathrm{PPh}_{2}\right)(\mathrm{Ph})\left(\mathrm{PPh}_{3}\right)_{3}\right]^{+}$cations and $\mathrm{BF}_{4}^{-}-$anions. The Pt(II) - Pt(II) bond distances are 2.889(2) $\AA$ (molecule 1) and 2.912(3) $\AA$ (molecule 2). In each of the molecules, the $\mathrm{Pt}(\mathrm{II})$ atoms are in essentially square - planar arrangement, one with $\mathrm{PtP}_{3} \mathrm{H}$ and the other with $\mathrm{PtP}_{2} \mathrm{HC}$ chromophores. 
Table 2. Crystallographic and Structural Data for Mononuclear Platinum Organometallics which Contain Crystallographic Independent Molecules ${ }^{\mathrm{a}}$

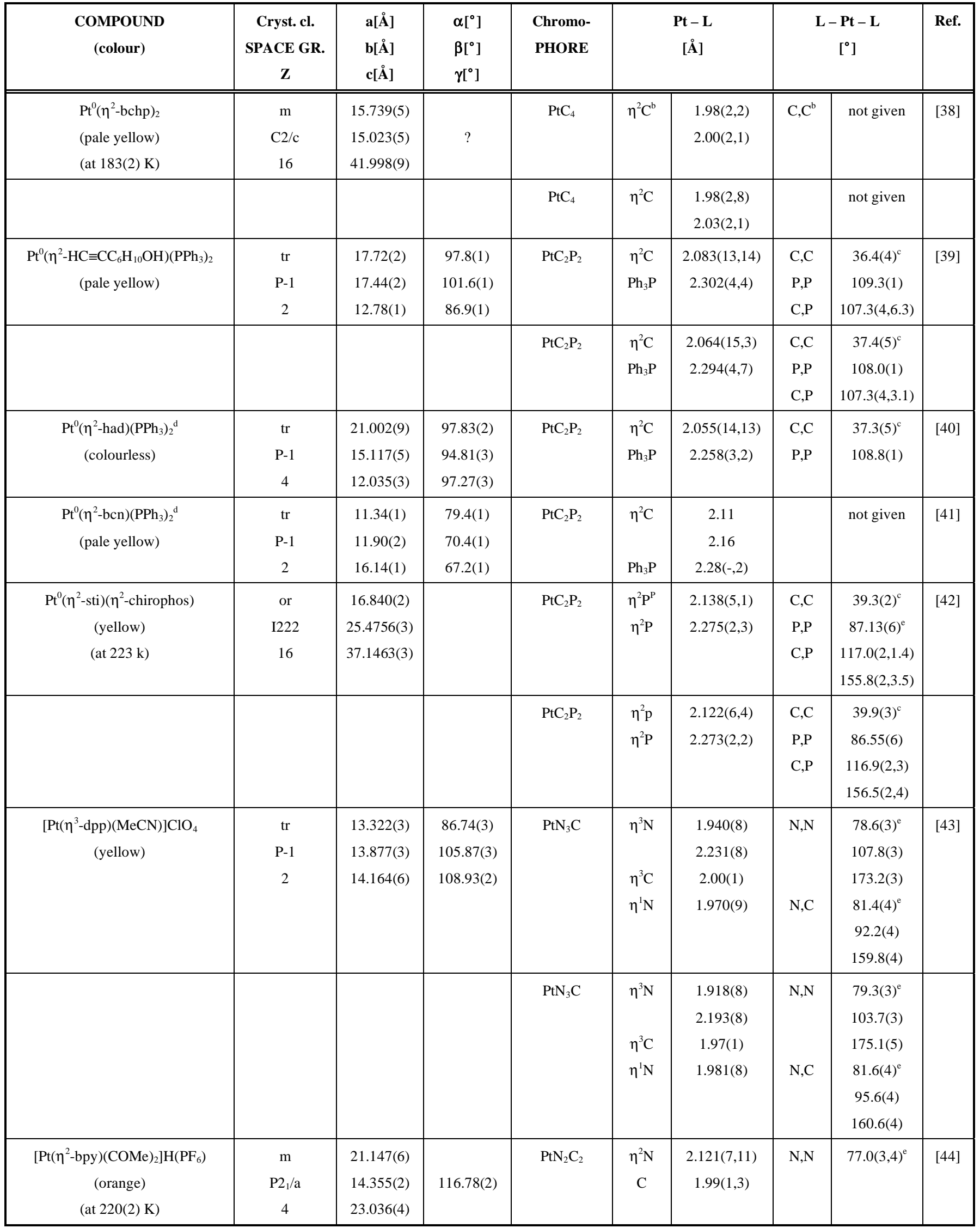


(Table 2).contd.....

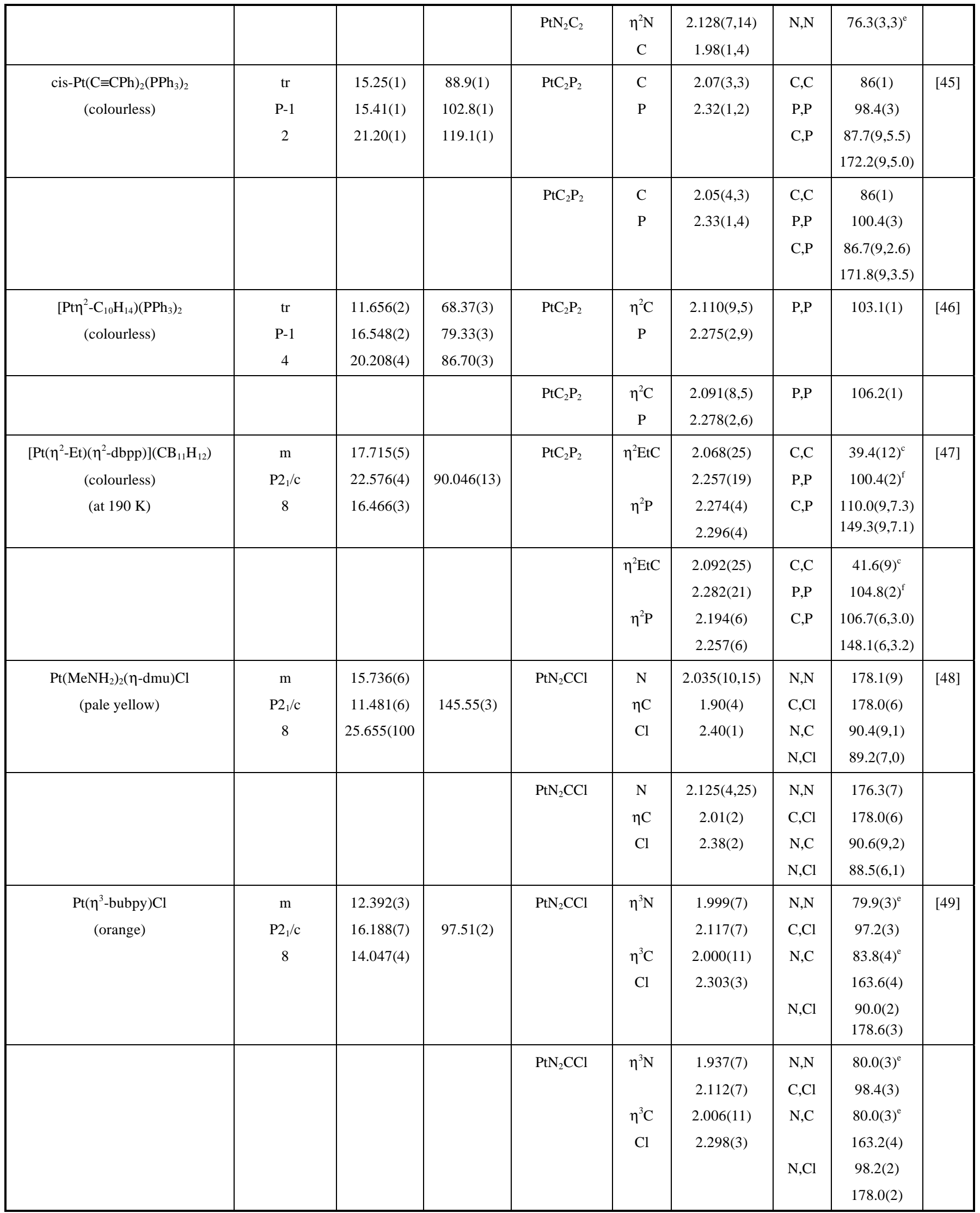




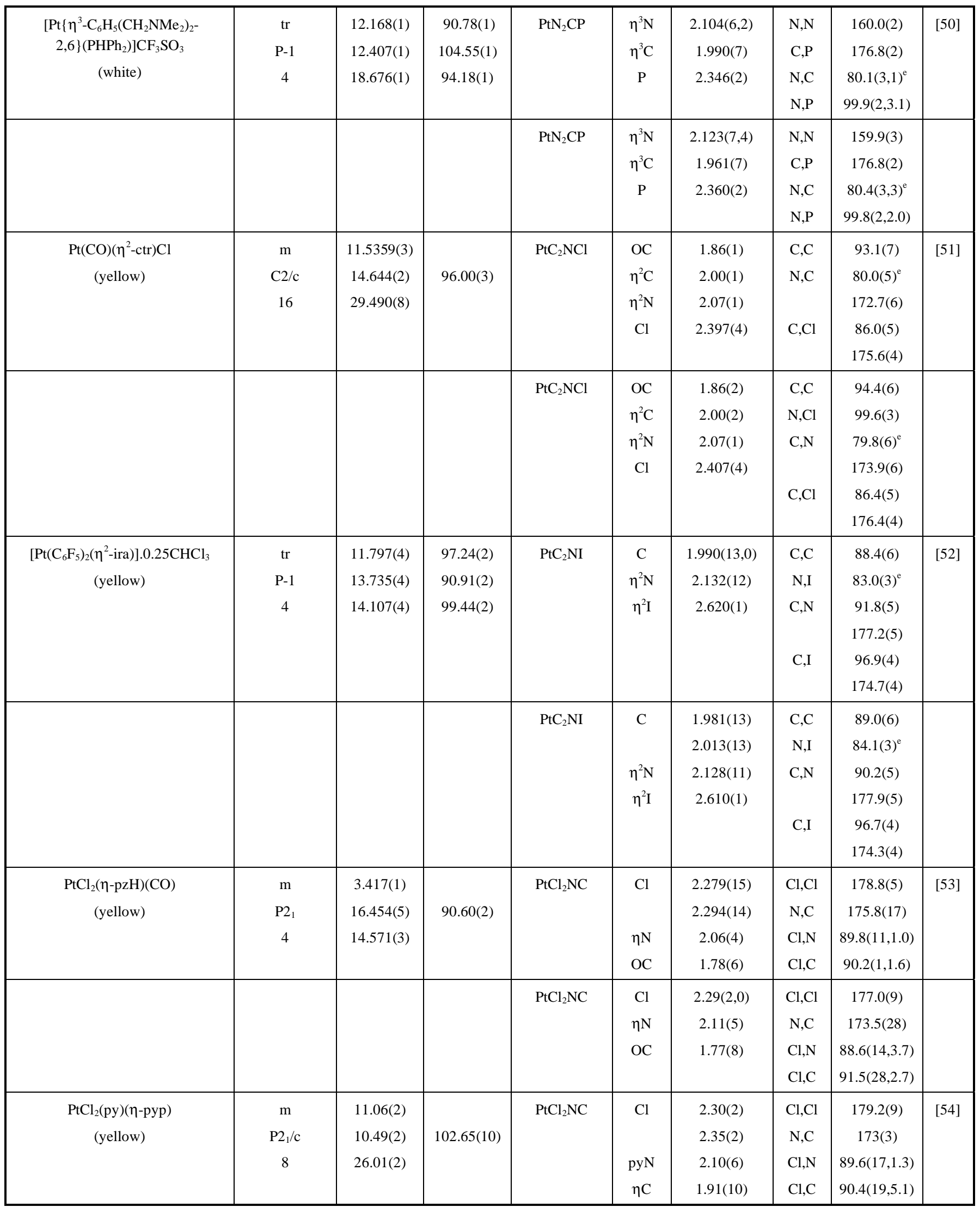


(Table 2).contd.....

\begin{tabular}{|c|c|c|c|c|c|c|c|c|c|}
\hline & & & & $\mathrm{PtCl}_{2} \mathrm{NC}$ & $\begin{array}{c}\mathrm{Cl} \\
\mathrm{pyN} \\
\eta \mathrm{C}\end{array}$ & $\begin{array}{l}2.30(2) \\
2.31(2) \\
2.05(5) \\
2.11(7)\end{array}$ & $\begin{array}{l}\mathrm{Cl}, \mathrm{Cl} \\
\mathrm{N}, \mathrm{C} \\
\mathrm{Cl}, \mathrm{N} \\
\mathrm{Cl}, \mathrm{C}\end{array}$ & $\begin{array}{c}178.3(8) \\
175(2) \\
90.3(15,1) \\
89.8(20,3.7)\end{array}$ & \\
\hline \multirow[t]{2}{*}{$\begin{array}{l}\mathrm{Pt}\left(\mathrm{SMe}_{2}\right)_{2}(\mathrm{Ph}) \mathrm{Cl} \\
\quad \text { (pale yellow) }\end{array}$} & $\begin{array}{c}\mathrm{m} \\
\mathrm{P} 2{ }_{1} / \mathrm{n} \\
8\end{array}$ & $\begin{array}{l}10.106(1) \\
13.046(3) \\
20.939(3)\end{array}$ & $94.39(1)$ & $\mathrm{PtS}_{2} \mathrm{CCl}$ & $\begin{array}{c}\mathrm{S} \\
\mathrm{PhC} \\
\mathrm{Cl}\end{array}$ & $\begin{array}{c}2.295(4,4) \\
1.99(1) \\
2.404(3)\end{array}$ & $\begin{array}{c}\mathrm{S}, \mathrm{S} \\
\mathrm{C}, \mathrm{Cl} \\
\mathrm{S}, \mathrm{C} \\
\mathrm{S}, \mathrm{Cl}\end{array}$ & $\begin{array}{l}170.8(1) \\
177.7(4) \\
85.7(4,3) \\
94.4(1,6)\end{array}$ & [12] \\
\hline & & & & $\mathrm{PtS}_{2} \mathrm{CCl}$ & $\begin{array}{c}\mathrm{S} \\
\mathrm{PhC} \\
\mathrm{Cl}\end{array}$ & $\begin{array}{c}2.287(4,8) \\
1.99(1) \\
2.403(4)\end{array}$ & $\begin{array}{c}\mathrm{S}, \mathrm{S} \\
\mathrm{C}, \mathrm{Cl} \\
\mathrm{S}, \mathrm{C} \\
\mathrm{S}, \mathrm{Cl}\end{array}$ & $\begin{array}{c}171.9(1) \\
177.2(3) \\
86.0(4,2) \\
94.1(1,1.1)\end{array}$ & \\
\hline $\begin{array}{l}\operatorname{Pt}\left(\operatorname{PPr}_{3}^{\mathrm{i}}\right)_{2}(\mathrm{H})(\mathrm{Ph}) \\
\quad(\text { colourless })\end{array}$ & & & & & $\begin{array}{c}\mathrm{P} \\
\mathrm{PhC}\end{array}$ & $\begin{array}{c}2.272(5,7) \\
2.05(2)\end{array}$ & $\begin{array}{l}\mathrm{P}, \mathrm{P} \\
\mathrm{P}, \mathrm{C}\end{array}$ & $\begin{array}{c}169.0(2) \\
95.5(5,1.1)\end{array}$ & \\
\hline \multirow[t]{2}{*}{$\begin{array}{c}\mathrm{Pt}\left(\mathrm{PMePh}_{2}\right)_{2}\left(\mathrm{C}_{2} \mathrm{~F}_{5}\right) \mathrm{Cl} \\
(\text { colourless })\end{array}$} & $\begin{array}{c}\text { or } \\
\text { Pna } 2_{1} \\
8\end{array}$ & $\begin{array}{c}15.4957(4) \\
9.983(3) \\
38.776(1)\end{array}$ & & $\mathrm{PtP}_{2} \mathrm{CCl}$ & $\begin{array}{l}\mathrm{P} \\
\mathrm{C} \\
\mathrm{Cl}\end{array}$ & $\begin{array}{c}2.335(3,6) \\
2.013(12) \\
2.361(3)\end{array}$ & $\begin{array}{c}\mathrm{P}, \mathrm{P} \\
\mathrm{C}, \mathrm{Cl} \\
\mathrm{P}, \mathrm{C} \\
\mathrm{P}, \mathrm{Cl}\end{array}$ & $\begin{array}{c}172.1(1) \\
177.0(4) \\
93.1(3,1.9) \\
87.1(3,2.9)\end{array}$ & [56] \\
\hline & & & & $\mathrm{PtP}_{2} \mathrm{CCl}$ & $\begin{array}{l}\mathrm{P} \\
\mathrm{C} \\
\mathrm{Cl}\end{array}$ & $\begin{array}{c}2.333(3,7) \\
1.990(12) \\
2.365(4)\end{array}$ & $\begin{array}{c}\mathrm{P}, \mathrm{P} \\
\mathrm{C}, \mathrm{Cl} \\
\mathrm{P}, \mathrm{C} \\
\mathrm{P}, \mathrm{Cl}\end{array}$ & $\begin{array}{c}171.9(1) \\
177.8(3) \\
92.9(4,2.7) \\
87.2(1,2.7)\end{array}$ & \\
\hline $\begin{array}{c}{\left[\mathrm{Pt}\left(\mathrm{PPh}_{3}\right)_{2}(\eta \text {-ipa }) \mathrm{Cl}\right] \cdot 2 / 3 \mathrm{CHCl}_{3}} \\
\text { (colourless) }\end{array}$ & & & & $\mathrm{PtP}_{2} \mathrm{CCl}$ & $\begin{array}{r}\mathrm{Ph}_{3} \mathrm{P} \\
\eta \mathrm{C} \\
\mathrm{Cl}\end{array}$ & $\begin{array}{c}2.298(3,2) \\
1.84(2) \\
2.361(6)\end{array}$ & $\begin{array}{c}\mathrm{P}, \mathrm{P} \\
\mathrm{C}, \mathrm{Cl} \\
\mathrm{P}, \mathrm{C} \\
\mathrm{P}, \mathrm{Cl}\end{array}$ & $\begin{array}{c}180 \\
180 \\
90.5(5,3.2) \\
89.6(2,1.0)\end{array}$ & \\
\hline \multirow[t]{2}{*}{$\begin{array}{c}\operatorname{Pt}\left(\mathrm{PPh}_{3}\right)\left(\eta^{2} \text {-mppc }\right) \mathrm{I} \\
\quad(\text { pale yellow })\end{array}$} & $\begin{array}{c}\mathrm{tr} \\
\mathrm{P}-1 \\
4\end{array}$ & $\begin{array}{l}10.507(2) \\
15.033(2) \\
25.523(8)\end{array}$ & $\begin{array}{l}77.06(2) \\
87.77(2) \\
70.29(2)\end{array}$ & $\mathrm{PtP}_{2} \mathrm{CI}$ & $\begin{array}{c}\mathrm{Ph}_{3} \mathrm{P} \\
\eta^{2} \mathrm{P} \\
\eta^{2} \mathrm{C} \\
\mathrm{I}\end{array}$ & $\begin{array}{c}2.310(4) \\
2.238(5) \\
2.07(2) \\
2.645(1)\end{array}$ & $\begin{array}{l}\mathrm{P}, \mathrm{P} \\
\mathrm{C}, \mathrm{I} \\
\mathrm{P}, \mathrm{C}\end{array}$ & $\begin{array}{c}102.4(2) \\
96.1(5) \\
67.7(5)^{\mathrm{g}} \\
170.1(5) \\
93.8(1) \\
163.4(1)\end{array}$ & [58] \\
\hline & & & & $\mathrm{PtP}_{2} \mathrm{CI}$ & $\begin{array}{c}\mathrm{Ph}_{3} \mathrm{P} \\
\eta^{2} \mathrm{P} \\
\eta^{2} \mathrm{C} \\
\mathrm{I}\end{array}$ & $\begin{array}{c}2.307(4) \\
2.228(4) \\
2.10(1) \\
2.641(1)\end{array}$ & $\begin{array}{l}\mathrm{P}, \mathrm{P} \\
\mathrm{C}, \mathrm{I} \\
\mathrm{P}, \mathrm{C}\end{array}$ & $\begin{array}{c}102.9(2) \\
96.6(4) \\
67.8(4)^{\mathrm{g}} \\
170.7(4) \\
92.7(1) \\
164.4(1)\end{array}$ & \\
\hline
\end{tabular}


(Table 2).contd......

\begin{tabular}{|c|c|c|c|c|c|c|c|c|c|}
\hline $\begin{array}{c}\operatorname{Pt}\left(\eta^{2}-\mathrm{mbpp}\right)(\mathrm{Cl})\left(\mathrm{PPr}_{3}\right)^{\mathrm{d}} \\
\text { (white) }\end{array}$ & $\begin{array}{c}\mathrm{m} \\
\mathrm{C} 2 / \mathrm{c} \\
8\end{array}$ & $\begin{array}{l}19.653(3) \\
11.538(2) \\
18.785(2)\end{array}$ & $107.10(1)$ & PtNCClP & $\begin{array}{c}\eta^{2} \mathrm{~N} \\
\eta^{2} \mathrm{C} \\
\mathrm{Cl} \\
\mathrm{Pr}_{3} \mathrm{P}\end{array}$ & $\begin{array}{l}2.179(9) \\
1.975(11) \\
2.406(3) \\
2.217(3)\end{array}$ & $\begin{array}{l}\mathrm{N}, \mathrm{C} \\
\mathrm{N}, \mathrm{Cl} \\
\mathrm{C}, \mathrm{P} \\
\mathrm{Cl}, \mathrm{P}\end{array}$ & $\begin{array}{c}69.0(4) \mathrm{g} \\
98.8(2) \\
96.6(3) \\
95.6(1)\end{array}$ & [59] \\
\hline $\begin{array}{c}\operatorname{Pt}\left(\eta^{2}-\text { ppy }\right)(\mathrm{Cl})\left(\mathrm{Pmor}_{3}\right)^{\mathrm{d}} \\
\text { (yellow) } \\
(\text { at } 93 \mathrm{~K})\end{array}$ & $\begin{array}{c}\mathrm{m} \\
\mathrm{P} 2{ }_{1} / \mathrm{a} \\
8\end{array}$ & $\begin{array}{l}17.192(5) \\
15.593(2) \\
18.178(4)\end{array}$ & $91.27(2)$ & PtNCCIP & $\begin{array}{c}\eta^{2} \mathrm{~N} \\
\eta^{2} \mathrm{C} \\
\mathrm{Cl} \\
\operatorname{mor}_{3} \mathrm{P}\end{array}$ & $\begin{array}{l}2.106(5) \\
2.001(9) \\
2.388(2) \\
2.233(2)\end{array}$ & $\begin{array}{c}\mathrm{N}, \mathrm{C} \\
\mathrm{N}, \mathrm{Cl} \\
\mathrm{N}, \mathrm{P} \\
\mathrm{C}, \mathrm{Cl} \\
\mathrm{C}, \mathrm{P} \\
\mathrm{Cl}, \mathrm{P}\end{array}$ & $\begin{array}{c}80.8(2)^{\mathrm{e}} \\
90.7(2) \\
174.6(2) \\
168.5(2) \\
98.0(2) \\
90.04(8)\end{array}$ & [60] \\
\hline $\begin{array}{c}\mathrm{Pt}(\mathrm{Me})\left(\eta^{2}-\mathrm{ptpc}\right) \mathrm{Cl}^{\mathrm{d}} \\
\quad(\text { colouless })\end{array}$ & $\begin{array}{c}\mathrm{tr} \\
\mathrm{P}-1 \\
4\end{array}$ & $\begin{array}{l}10.301(4) \\
10.986(5) \\
14.976(7)\end{array}$ & $\begin{array}{l}98.67(6) \\
92.90(6) \\
96.78(6)\end{array}$ & PtCClSP & $\begin{array}{c}\mathrm{MeC} \\
\mathrm{Cl} \\
\eta^{2} \mathrm{~S} \\
\eta^{2} \mathrm{P}\end{array}$ & $\begin{array}{l}2.084(7) \\
2.365(2) \\
2.374(2) \\
2.191(2)\end{array}$ & $\begin{array}{l}\mathrm{C}, \mathrm{Cl} \\
\mathrm{C}, \mathrm{S} \\
\mathrm{C}, \mathrm{P} \\
\mathrm{Cl}, \mathrm{S} \\
\mathrm{Cl} . \mathrm{P} \\
\mathrm{S}, \mathrm{P}\end{array}$ & $\begin{array}{c}89.9(2) \\
177.7(2) \\
94.3(3) \\
88.9(1) \\
175.6(1) \\
87.0(1)^{\mathrm{g}}\end{array}$ & [61] \\
\hline $\begin{array}{c}\mathrm{Pt}\left(\eta^{3}-\mathrm{dmps}\right) \mathrm{Cl}_{2} \\
\quad(\text { colouless })\end{array}$ & $\begin{array}{c}\mathrm{m} \\
\mathrm{P} 2 / \mathrm{c} \\
8\end{array}$ & $\begin{array}{l}12.831(1) \\
12.950(1) \\
16.206(2)\end{array}$ & $108.14(1)$ & $\mathrm{PtC}_{3} \mathrm{Cl}_{2}$ & $\begin{array}{l}\eta^{3} \mathrm{C} \\
\mathrm{Cl}\end{array}$ & $\begin{array}{c}1.99(2) \\
2.13(3,2) \\
2.286(7) \\
2.386(3)\end{array}$ & $\begin{array}{l}\mathrm{C}, \mathrm{C} \\
\mathrm{Cl}, \mathrm{Cl} \\
\mathrm{C}, \mathrm{Cl}\end{array}$ & $\begin{array}{c}36.7(7)^{\mathrm{c}} \\
92(1,1) \\
90.6(3) \\
88.3(7,2.1) \\
161.5(7,1.2) \\
177.3(6)\end{array}$ & [37] \\
\hline & & & & $\mathrm{PtC}_{3} \mathrm{Cl}_{2}$ & $\begin{array}{l}\eta^{3} \mathrm{C} \\
\mathrm{Ph}_{3} \mathrm{P}\end{array}$ & $\begin{array}{l}2.102(1) \\
2.15(2,3) \\
2.323(5) \\
2.407(2)\end{array}$ & $\begin{array}{l}\mathrm{C}, \mathrm{C} \\
\mathrm{Cl}, \mathrm{Cl} \\
\mathrm{C}, \mathrm{Cl}\end{array}$ & $\begin{array}{c}40.1(7)^{\mathrm{c}} \\
92.9(8,1.4) \\
91.1(2) \\
87.9(6,1.6) \\
160.0(6,4.2) \\
179.4(6)\end{array}$ & \\
\hline $\begin{array}{c}{\left[\mathrm{Pt}\left\{\eta^{2}-\mathrm{CH}_{2} \mathrm{C}\left(\mathrm{C}\left(\mathrm{CO}_{2} \mathrm{Me}\right)_{2}\right)\right.\right.} \\
\left.\mathrm{CHPh}\}\left(\mathrm{PPh}_{3}\right)_{2}\right] \text { tol.0.5thf } \\
\text { (pale yellow) }\end{array}$ & $\begin{array}{c}\mathrm{m} \\
\mathrm{P} 2 / \mathrm{n} \\
4\end{array}$ & $\begin{array}{l}12.750(2) \\
18.503(4) \\
39.658(6)\end{array}$ & $94.69(1)$ & $\mathrm{PtC}_{3} \mathrm{Cl}_{2}$ & $\begin{array}{l}\eta^{3} \mathrm{C} \\
\mathrm{Ph}_{3} \mathrm{P}\end{array}$ & $\begin{array}{c}2.136(11,10) \\
2.348(10) \\
2.2709(3) \\
2.307(2)\end{array}$ & $\begin{array}{l}\mathrm{C}, \mathrm{C} \\
\mathrm{P}, \mathrm{P} \\
\mathrm{C}, \mathrm{P}\end{array}$ & $\begin{array}{c}37.1(4,4)^{\mathrm{c}} \\
65.9(4) \\
100.31(9) \\
97.0(3,3.3) \\
127.7(3,4.4) \\
162.6(3,3.2)\end{array}$ & [62] \\
\hline & & & & $\mathrm{PtC}_{3} \mathrm{P}_{2}$ & $\begin{array}{l}\eta^{2} \mathrm{C} \\
\mathrm{Ph}_{3} \mathrm{P}\end{array}$ & $\begin{array}{c}2.148(10,2) \\
2.352(9) \\
2.280(3) \\
2.311(2)\end{array}$ & $\begin{array}{l}\mathrm{C}, \mathrm{C} \\
\mathrm{P}, \mathrm{P} \\
\mathrm{C}, \mathrm{P}\end{array}$ & $\begin{array}{c}37.6(3,2)^{\mathrm{c}} \\
66.3(2) \\
99.19(9) \\
97.3(3,3.2) \\
127.7(2,3.7) \\
163.5(3,3.3)\end{array}$ & \\
\hline $\begin{array}{c}\mathrm{Pt}\left(\eta^{2}-\mathrm{CH}_{2}=\mathrm{CH}_{2}\right)(\mathrm{Cl})_{2}\left(\eta^{1}-\mathrm{eox}\right) \\
\text { (yellow) }\end{array}$ & $\begin{array}{c}\text { or } \\
\mathrm{P} 22_{1} 2_{1} \\
8\end{array}$ & $\begin{array}{l}7.003(1) \\
16.573(1) \\
29.088(4)\end{array}$ & & $\mathrm{PtC}_{2} \mathrm{Cl}_{2} \mathrm{~N}$ & $\begin{array}{c}\eta^{2} \mathrm{C} \\
\mathrm{Cl} \\
\eta^{1} \mathrm{~N}\end{array}$ & $\begin{array}{c}2.165(20,5) \\
2.282(6,1) \\
2.10(1)\end{array}$ & $\begin{array}{l}\mathrm{Cl}, \mathrm{Cl} \\
\mathrm{Cl}, \mathrm{N}\end{array}$ & $\begin{array}{c}177.6(2) \\
88.9(4,1.7)\end{array}$ & [63] \\
\hline & & & & $\mathrm{PtC}_{2} \mathrm{Cl}_{2} \mathrm{~N}$ & $\begin{array}{c}\eta^{2} \mathrm{C} \\
\mathrm{Cl} \\
\eta^{1} \mathrm{~N}\end{array}$ & $\begin{array}{c}2.18(2,3) \\
2.288(6,1) \\
2.10(1)\end{array}$ & $\begin{array}{l}\mathrm{Cl}, \mathrm{Cl} \\
\mathrm{Cl}, \mathrm{N}\end{array}$ & $\begin{array}{c}177.3(2) \\
88.8(4,2.5)\end{array}$ & \\
\hline
\end{tabular}


(Table 2).contd.....

\begin{tabular}{|c|c|c|c|c|c|c|c|c|c|}
\hline $\begin{array}{c}\operatorname{Pt}\left(\eta^{4}-\operatorname{cod}\right)\left(\eta^{2}-\text { metu }\right) \\
\text { (pale yellow) } \\
(\text { at } 130 \mathrm{~K})\end{array}$ & $\begin{array}{c}\mathrm{m} \\
\mathrm{P} 2 / \mathrm{c} \\
8\end{array}$ & $\begin{array}{l}11.270(3) \\
12.268(2) \\
17.053(4)\end{array}$ & $96.00(2)$ & $\mathrm{PtC}_{4} \mathrm{NS}$ & $\begin{array}{l}\eta^{4} \mathrm{C} \\
\eta^{2} \mathrm{~N} \\
\eta^{2} \mathrm{~S}\end{array}$ & $\begin{array}{c}2.16(1,1) \\
2.20(1,1) \\
2.00(1) \\
2.318(3)\end{array}$ & $\begin{array}{l}\mathrm{C}, \mathrm{C} \\
\mathrm{N}, \mathrm{S} \\
\mathrm{C}, \mathrm{N} \\
\\
\mathrm{C}, \mathrm{S}\end{array}$ & $\begin{array}{c}82.0(5,6) \\
70.7(3)^{\mathrm{g}} \\
99.6(5,4) \\
156.3(4) \\
101.6(3,1.6)\end{array}$ & [64] \\
\hline & & & & $\mathrm{PtC}_{4} \mathrm{NS}$ & $\begin{array}{l}\eta^{4} \mathrm{C} \\
\eta^{2} \mathrm{~N} \\
\eta^{2} \mathrm{~S}\end{array}$ & $\begin{array}{c}2.17(1,0) \\
2.19(1,1) \\
2.01(1) \\
2.303(3)\end{array}$ & $\begin{array}{l}\mathrm{C}, \mathrm{C} \\
\mathrm{N}, \mathrm{S} \\
\mathrm{C}, \mathrm{N} \\
\\
\mathrm{C}, \mathrm{S}\end{array}$ & $\begin{array}{c}81.4(5,1) \\
70.7(3)^{\mathrm{g}} \\
100.8(4,1.1) \\
155.7(5) \\
100.9(3,2.1)\end{array}$ & \\
\hline $\begin{array}{c}{\left[\mathrm{Pt}\left(\eta^{2}-\mathrm{dmphen}\right)\left(\eta^{2}-\right.\right.} \\
\left.\mathrm{PhC} \equiv \mathrm{CPh}) \mathrm{I}_{2}\right] \cdot \mathrm{CHCl}_{3} \\
\quad(\text { dark red })\end{array}$ & $\begin{array}{c}\text { or } \\
\mathrm{Cmc} 2_{1} \\
8\end{array}$ & $\begin{array}{l}14.367(3) \\
15.529(3) \\
26.959(5)\end{array}$ & & $\mathrm{PtN}_{2} \mathrm{C}_{2} \mathrm{I}_{2}$ & $\begin{array}{c}\eta^{2} \mathrm{~N} \\
\eta^{2} \mathrm{C} \\
\mathrm{I}\end{array}$ & $\begin{array}{c}2.28(2,0) \\
2.01(2,0) \\
2.647(3,22)\end{array}$ & $\begin{array}{c}\mathrm{N}, \mathrm{N} \\
\mathrm{C}, \mathrm{C} \\
\mathrm{I}, \mathrm{I} \\
\mathrm{N}, \mathrm{C} \\
\mathrm{N}, \mathrm{I} \\
\mathrm{C}, \mathrm{I}\end{array}$ & $\begin{array}{c}71.9(6)^{\mathrm{e}} \\
36.1(8) \\
178.7(1) \\
125.7(7) \\
89.5(5,3.2) \\
90.6(6,2.8)\end{array}$ & [65] \\
\hline & & & & $\mathrm{PtN}_{2} \mathrm{C}_{2} \mathrm{I}_{2}$ & $\begin{array}{c}\eta^{2} \mathrm{~N} \\
\eta^{2} \mathrm{C} \\
\mathrm{I}\end{array}$ & $\begin{array}{c}2.22(2,0) \\
2.08(3,0) \\
2.644(3,28)\end{array}$ & $\begin{array}{c}\mathrm{N}, \mathrm{N} \\
\mathrm{C}, \mathrm{C} \\
\mathrm{I}, \mathrm{I} \\
\mathrm{N}, \mathrm{C} \\
\mathrm{N}, \mathrm{I} \\
\mathrm{C}, \mathrm{I}\end{array}$ & $\begin{array}{c}74.9(7)^{\mathrm{e}} \\
35.5(10)^{\mathrm{c}} \\
179.5(1) \\
124.8(9) \\
90.2(5,4) \\
89.8(7,2)\end{array}$ & \\
\hline $\begin{array}{c}{\left[\mathrm{Pt}^{\mathrm{IV}}\left(\eta^{2}-\mathrm{bpy}\right)\left(\eta^{2}-\mathrm{mq}\right)\right.} \\
\left.(\mathrm{Me})_{2}\right] \mathrm{BF}_{4} \cdot 0.75 \mathrm{CH}_{2} \mathrm{Cl}_{2} \\
\text { (white) }\end{array}$ & $\begin{array}{c}\mathrm{m} \\
\mathrm{C} 2 / \mathrm{c} \\
16\end{array}$ & $\begin{array}{l}40.624(4) \\
12.890(1) \\
19.765(2)\end{array}$ & $91.347(2)$ & $\mathrm{PtN}_{3} \mathrm{C}_{3}$ & $\begin{array}{l}\eta^{2} \mathrm{~N} \\
\eta^{2} \mathrm{~N} \\
\eta^{2} \mathrm{C} \\
\mathrm{MeC}\end{array}$ & $\begin{array}{c}2.15(1,1) \\
2.151(9) \\
2.05(1) \\
2.05(1,2)\end{array}$ & $\begin{array}{l}\mathrm{N}, \mathrm{N} \\
\mathrm{C}, \mathrm{C} \\
\mathrm{N}, \mathrm{C}\end{array}$ & $\begin{array}{c}76.1(4)^{\mathrm{e}} \\
89.4(6,2.0) \\
82.6(5)^{\mathrm{e}} \\
96.8(5) \\
172.8(5,2)\end{array}$ & [66] \\
\hline $\begin{array}{c}\left.\left[\mathrm{Pt}^{\mathrm{IV}}\left(\mathrm{\eta}^{3}-\mathrm{htpzb}\right)(\mathrm{Ph})_{2} \mathrm{I}\right)\right]_{2}(\mathrm{I})\left(\mathrm{I}_{3}\right) \\
\text { (black) }\end{array}$ & $\begin{array}{c}\mathrm{tr} \\
\mathrm{P}-1 \\
4\end{array}$ & $\begin{array}{r}18.614(4) \\
13.114(9) \\
11.794(11)\end{array}$ & $\begin{array}{l}78.14(7) \\
86.69(7) \\
88.24(4)\end{array}$ & $\mathrm{PtN}_{3} \mathrm{C}_{2} \mathrm{I}$ & $\begin{array}{c}\eta^{3} \mathrm{~N} \\
\\
\\
\mathrm{PhC} \\
\mathrm{I}\end{array}$ & $\begin{array}{c}2.01(2) \\
2.15(2) \\
2.20(2) \\
2.07(3,2) \\
2.601(3)\end{array}$ & $\begin{array}{l}\mathrm{N}, \mathrm{N} \\
\mathrm{C}, \mathrm{C} \\
\mathrm{N}, \mathrm{C} \\
\mathrm{N}, \mathrm{I} \\
\\
\mathrm{C}, \mathrm{I}\end{array}$ & $\begin{array}{c}84.5(9,6)^{\mathrm{e}} \\
98(1) \\
89(1,1) \\
177(1,0) \\
91.4(6,6) \\
174.8(5) \\
93.9(9,1)\end{array}$ & [67] \\
\hline & & & & $\mathrm{PtN}_{3} \mathrm{C}_{2} \mathrm{I}$ & $\begin{array}{r}\eta^{3} \mathrm{~N} \\
\mathrm{PhC} \\
\\
\mathrm{I}\end{array}$ & $\begin{array}{c}2.11(3,1) \\
2.18(3) \\
2.03(3) \\
2.43(3) \\
2.63(4)\end{array}$ & $\begin{array}{l}\mathrm{N}, \mathrm{N} \\
\mathrm{C}, \mathrm{C} \\
\mathrm{N}, \mathrm{C} \\
\mathrm{N}, \mathrm{I} \\
\mathrm{C}, \mathrm{I}\end{array}$ & $\begin{array}{c}82(1,2)^{\mathrm{e}} \\
100(2) \\
93(1,1) \\
173(1) \\
96.2(2,4.5) \\
172.9(8) \\
95(2,4)\end{array}$ & \\
\hline
\end{tabular}


(Table 2).contd.....

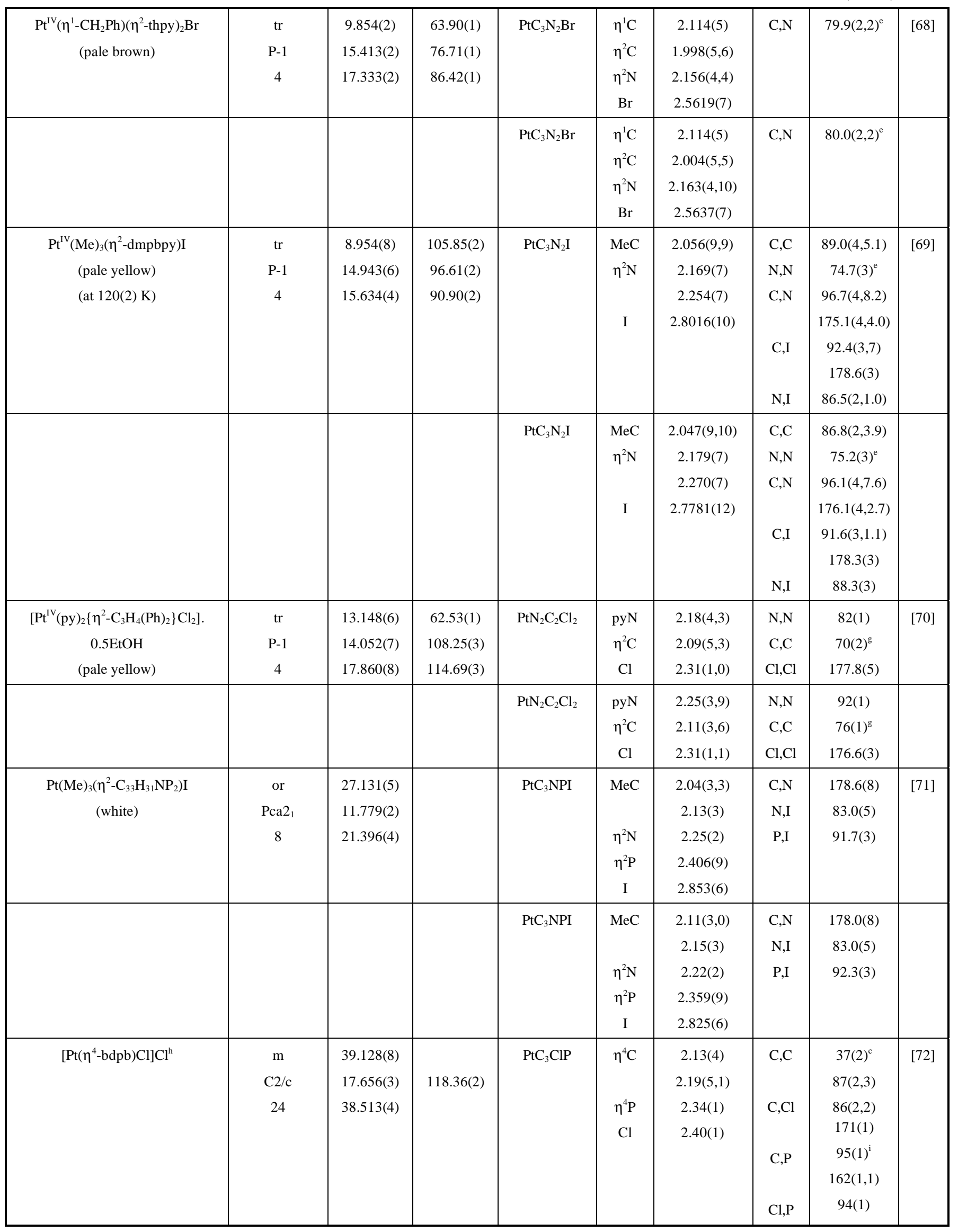


(Table 2).contd.....

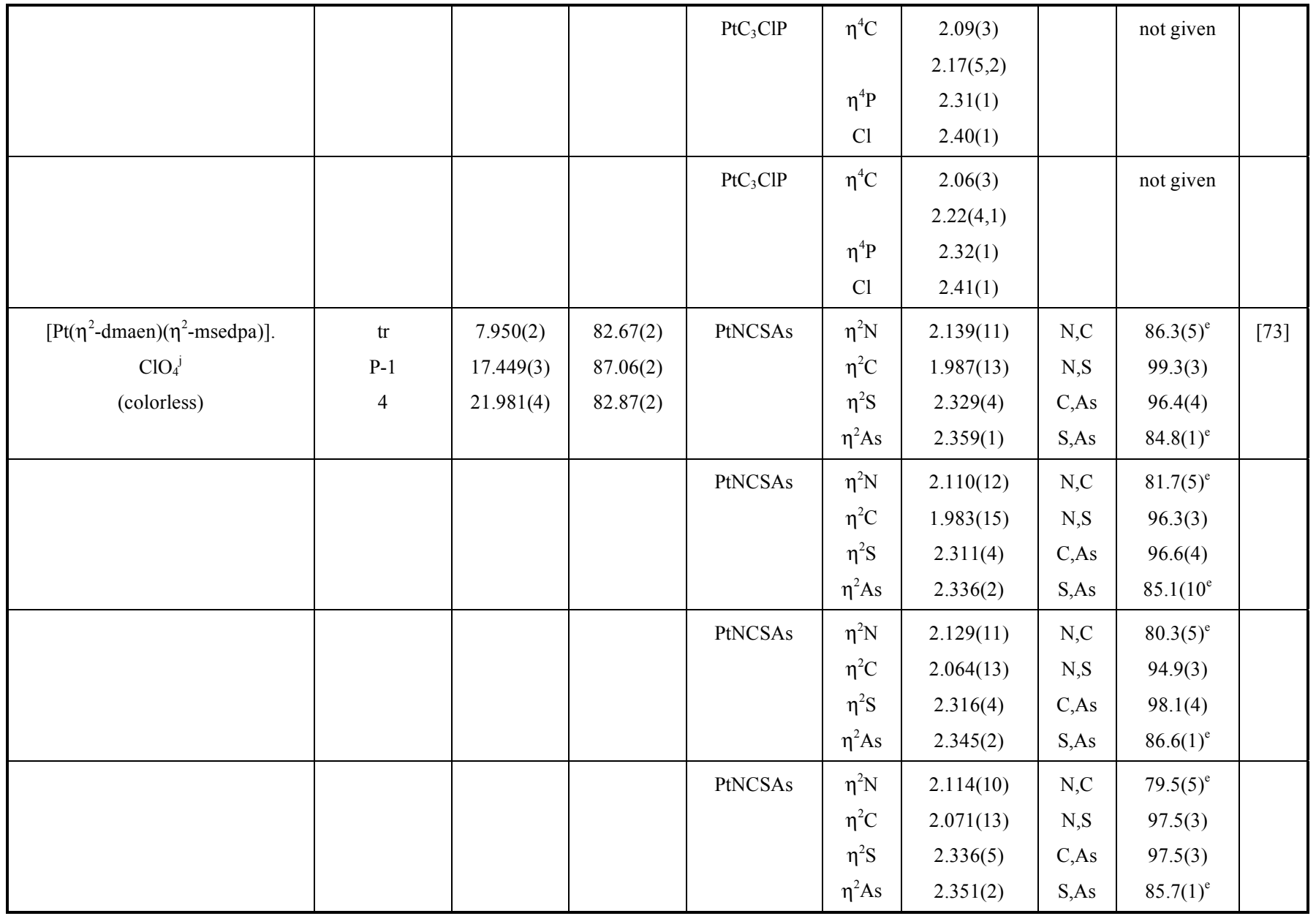

Footnotes: a.) Where more than one chemically equivalent distance or angle is present, the mean value is tabulated. The first number in parenthesis is the e.s.d., and the second is the maximum deviation from the mean.

b). The chemical identity of the coordinated atom or ligand is specified in these columns

c). Three - membered metallocyclic ring.

d). Mean values for both molecules are given in original paper.

e). Five-membered metallocyclic ring.

f). Six - membered metallocyclic ring.

g). Four - membered metallocyclic ring.

h). Three crystallographically independent molecules.

i). Nine - membered metallocyclic ring.

j). Four crystallographically independent molecules.

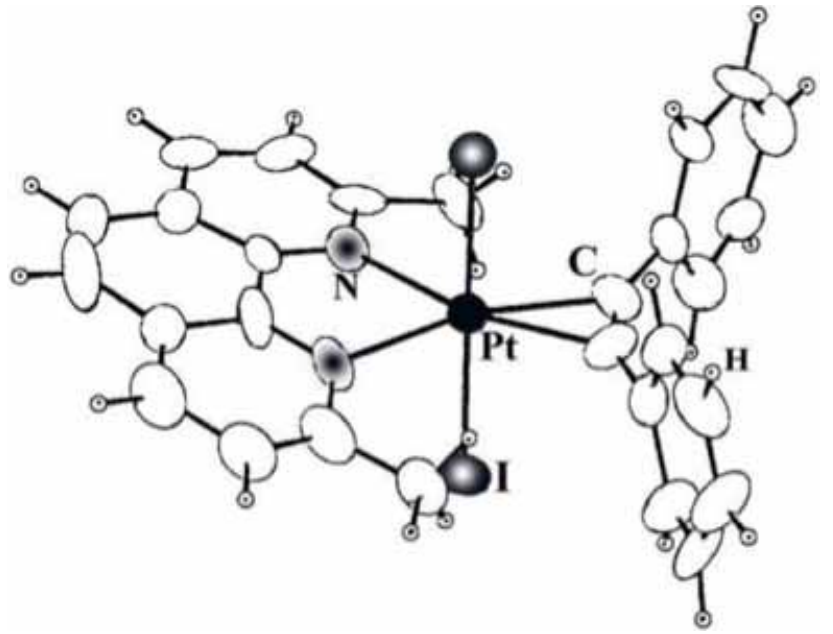

Fig. (2). Structure of one isomeric form of $\left[\mathrm{Pt}\left(\eta^{2}-\mathrm{PhC} \equiv \mathrm{CPh}\right)\left(\eta^{2}-\right.\right.$ dmphen) $\left.\mathrm{I}_{2}\right] . \mathrm{CHCl}_{3}[65]$.

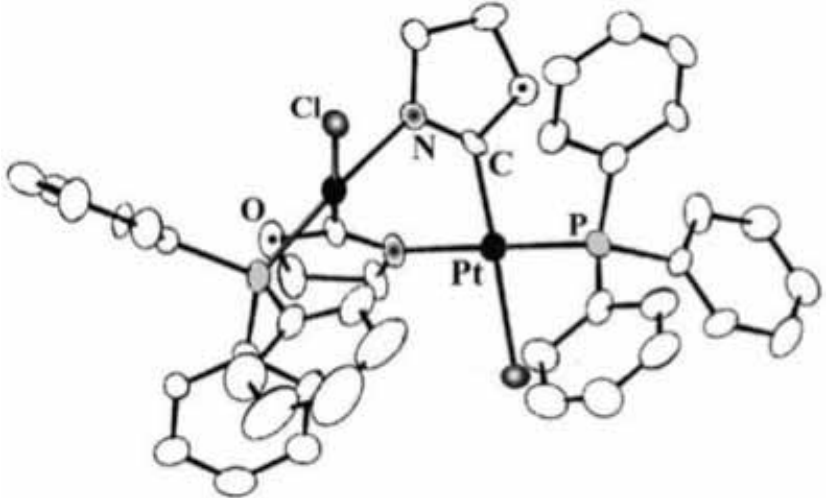

Fig. (3). Structure of one independent molecule of $\left[\operatorname{Pt}\left(\mu-\eta^{2}-\right.\right.$ $\left.\left.\mathrm{COCH}_{2} \mathrm{CH}_{2} \mathrm{~N}\right)(\mathrm{Cl})\left(\mathrm{PPh}_{3}\right)\right]_{2}$ [79].

In yellow $\mathrm{Pt}_{2}(\mathrm{Me})_{6}(\mu \text {-salal })_{2}$ [77] dimerisation occurs by formation of a four - membered centrosymmetrical $\mathrm{Pt}(\mathrm{O})_{2} \mathrm{Pt}$ ring via the phenolic oxygen. Around each Pt(IV) atom there 
Table 3. Crystallographic and Structural Data for Bi-, Tri- and Tetra-nuclear Platinum Organometallic Compounds which Contain Two Crystallographically Independent Molecules ${ }^{\mathrm{a}}$

\begin{tabular}{|c|c|c|c|c|c|c|c|c|c|c|}
\hline $\begin{array}{l}\text { COMPOUND } \\
\text { (colour) }\end{array}$ & $\begin{array}{c}\text { Cryst.cl. } \\
\text { Space GR. } \\
\text { Z }\end{array}$ & $\begin{array}{l}\mathrm{a}[\mathbf{\AA}] \\
\mathrm{b}[\AA ̊ \AA] \\
\mathrm{c}[\AA \mathbf{\AA}]\end{array}$ & $\begin{array}{l}\alpha\left[^{\circ}\right] \\
\beta\left[^{\circ}\right] \\
\gamma\left[^{\circ}\right]\end{array}$ & $\begin{array}{l}\text { Chromo- } \\
\text { PHORE }\end{array}$ & & $\begin{array}{l}\mathbf{P t}-\mathbf{L} \\
{[\AA ̊}\end{array}$ & $\begin{array}{c}\mathbf{P t}-\mathbf{P t}\left[\left[^{\mathbf{A}}\right]\right. \\
\mathbf{P t}-\mathbf{L}-\mathbf{P t}\left[^{\circ}\right]\end{array}$ & \multicolumn{2}{|c|}{$\begin{array}{c}\mathbf{L}-\mathbf{P t}-\mathbf{L} \\
{\left[{ }^{\circ}\right]}\end{array}$} & Ref. \\
\hline \multicolumn{11}{|l|}{ A. BINUCLEAR } \\
\hline \multirow[t]{2}{*}{$\begin{array}{c}\mathrm{Pt}_{2}(\mu-\mathrm{S})(\mathrm{CO})_{2}\left(\mathrm{PPh}_{3}\right)_{2} \\
\text { (yellow) }\end{array}$} & $\begin{array}{c}\mathrm{m} \\
\mathrm{P} 2{ }_{1} / \mathrm{n} \\
4\end{array}$ & $\begin{array}{l}17.997(3) \\
20.620(7) \\
19.078(4)\end{array}$ & $98.28(2)$ & $\mathrm{Pt}^{\mathrm{I}} \mathrm{CSP}$ & $\begin{array}{c}\mathrm{OC}^{\mathrm{b}} \\
\mu \mathrm{S} \\
\mathrm{Ph}_{3} \mathrm{P}\end{array}$ & $\begin{array}{c}1.82(2,6) \\
2.264(5,6) \\
2.292(4,0)\end{array}$ & $\begin{array}{l}2.5998(9) \\
\text { S 70.1(1) }\end{array}$ & $\begin{array}{l}\mathrm{C}, \mathrm{S}^{\mathrm{b}} \\
\mathrm{C}, \mathrm{P} \\
\mathrm{S}, \mathrm{P}\end{array}$ & $\begin{array}{c}153.9(7,3) \\
100.3(5,2.3) \\
105.2(2,2.6)\end{array}$ & [74] \\
\hline & & & & $\mathrm{Pt}^{\mathrm{I}} \mathrm{CSP}$ & $\begin{array}{c}\mathrm{OC} \\
\mu \mathrm{S} \\
\mathrm{Ph}_{3} \mathrm{P}\end{array}$ & $\begin{array}{c}1.87(3,2) \\
2.262(5,8) \\
2.293(4,6)\end{array}$ & $\begin{array}{l}2.6008(8) \\
\text { S 70.2(1) }\end{array}$ & $\begin{array}{l}\mathrm{C}, \mathrm{S} \\
\mathrm{C}, \mathrm{P} \\
\mathrm{S}, \mathrm{P}\end{array}$ & $\begin{array}{c}155.1(8,4) \\
101.2(6,1.2) \\
105.2(2,2.6)\end{array}$ & \\
\hline \multirow[t]{3}{*}{$\begin{array}{c}\mathrm{Pt}_{2}(\mu-\mathrm{C} \equiv \mathrm{CHPh})\left(\mathrm{PEt}_{3}\right)_{3} \cdot \mathrm{Br}_{2} \\
\text { (pale orange) }\end{array}$} & $\begin{array}{l}\mathrm{m} \\
\mathrm{Cc} \\
8\end{array}$ & $\begin{array}{l}10.359(1) \\
33.054(9) \\
19.855(5)\end{array}$ & $93.40(2)$ & $\mathrm{PtCPBr}$ & $\begin{array}{c}\mu \mathrm{C} \\
\mathrm{Et}_{3} \mathrm{P} \\
\mathrm{Br}\end{array}$ & $\begin{array}{c}1.94(2) \\
2.200(4) \\
2.521(3)\end{array}$ & $\begin{array}{l}2.682(1) \\
\text { C } 84.6(6)\end{array}$ & $\begin{array}{l}\mathrm{C}, \mathrm{P} \\
\mathrm{C}, \mathrm{Br} \\
\mathrm{P}, \mathrm{Br}\end{array}$ & $\begin{array}{l}110.3(5) \\
157.9(5) \\
91.8(2,8)\end{array}$ & [75] \\
\hline & & & & $\mathrm{PtCPBr}$ & $\begin{array}{c}\mu \mathrm{C} \\
\mathrm{Et}_{3} \mathrm{P} \\
\mathrm{Br}\end{array}$ & $\begin{array}{c}1.85(1) \\
2.216(5) \\
2.543(3)\end{array}$ & $\begin{array}{l}2.687(1) \\
\text { C } 85.8(6)\end{array}$ & $\begin{array}{l}\text { C.P } \\
\text { C,Br } \\
\mathrm{P}, \mathrm{Br}\end{array}$ & $\begin{array}{c}109.3(5) \\
160.1(5) \\
90.5(2)\end{array}$ & \\
\hline & & & & $\mathrm{PtP}_{2} \mathrm{CBr}$ & $\begin{array}{c}\mathrm{Et}_{3} \mathrm{P} \\
\mu \mathrm{C} \\
\mathrm{Br}\end{array}$ & $\begin{array}{c}2.321(6,16) \\
2.09(2) \\
2.491(3)\end{array}$ & & $\begin{array}{c}\mathrm{P}, \mathrm{P} \\
\mathrm{C}, \mathrm{Br} \\
\mathrm{P}, \mathrm{Br}\end{array}$ & $\begin{array}{c}177.0(2) \\
174.5(5) \\
89.6(2,1.2)\end{array}$ & \\
\hline \multirow[t]{2}{*}{$\begin{array}{c}\mathrm{Pt}_{2}(\mathrm{Me})_{6}(\mu-\mathrm{salal})_{2} \\
\text { (yellow) }\end{array}$} & $\begin{array}{c}\operatorname{tr} \\
\mathrm{P}-1 \\
4\end{array}$ & $\begin{array}{c}10.892(5) \\
11.455(5) \\
9.147(5)\end{array}$ & $\begin{array}{c}74.7(5) \\
110.8(5) \\
92.55(5)\end{array}$ & $\mathrm{Pt}^{\mathrm{IV}} \mathrm{O}_{3} \mathrm{C}_{3}$ & $\begin{array}{c}\mu \mathrm{O} \\
\mathrm{O} \\
\mathrm{MeC}\end{array}$ & $\begin{array}{c}2.24(3,1) \\
2.25(4) \\
1.96(4) \\
2.11(4,2)\end{array}$ & $\begin{array}{l}3.407(4) \\
\text { O } 100(1)\end{array}$ & $\begin{array}{c}\mu \mathrm{O}, \mu \mathrm{O} \\
\mu \mathrm{O}, \mathrm{O} \\
\mathrm{C}, \mathrm{C}\end{array}$ & $\begin{array}{c}80(1) \\
88(1,2) \\
89(2,1)\end{array}$ & [77] \\
\hline & & & & $\mathrm{Pt}^{\mathrm{IV}} \mathrm{O}_{3} \mathrm{C}_{3}$ & $\begin{array}{c}\mu \mathrm{O} \\
\mathrm{O} \\
\mathrm{MeC}\end{array}$ & $\begin{array}{c}2.20(3,3) \\
2.27(3) \\
1.95(5) \\
2.04(5,2)\end{array}$ & $\begin{array}{l}3.418(4) \\
\text { O } 102(1)\end{array}$ & $\begin{array}{c}\mu \mathrm{O}, \mu \mathrm{O} \\
\mu \mathrm{O}, \mathrm{O} \\
\mathrm{C}, \mathrm{C}\end{array}$ & $\begin{array}{c}78(1) \\
87(1,2) \\
87(2,4)\end{array}$ & \\
\hline $\begin{array}{c}\mathrm{Pt}_{2}\left(\mu-\mathrm{NH}_{2}\right)_{2}(\mathrm{Me})_{2}\left(\mathrm{PPh}_{3}\right)_{2} \\
\text { (colourless) }\end{array}$ & $\begin{array}{c}\mathrm{m} \\
\mathrm{C} 2 / \mathrm{c} \\
8\end{array}$ & $\begin{array}{l}22.592(5) \\
11.844(3) \\
29.403(6)\end{array}$ & $116.43(2)$ & $\mathrm{PtN}_{2} \mathrm{CP}$ & $\begin{array}{c}\mu \mathrm{N} \\
\mathrm{MeC} \\
\mathrm{Ph}_{3} \mathrm{P}\end{array}$ & $\begin{array}{l}2.13(1,0) \\
2.07(1) \\
2.205(2)\end{array}$ & $\begin{array}{l}3.106(1) \\
\text { N 95.2(4) }\end{array}$ & $\begin{array}{l}\mathrm{N}, \mathrm{N} \\
\mathrm{C}, \mathrm{P} \\
\mathrm{N}, \mathrm{C} \\
\\
\mathrm{N}, \mathrm{P}\end{array}$ & $\begin{array}{c}77.5(4) \\
89.1(3) \\
90.4(4) \\
167.7(3) \\
102.9(2)\end{array}$ & [78] \\
\hline
\end{tabular}


(Table 3).contd.....

\begin{tabular}{|c|c|c|c|c|c|c|c|c|c|c|}
\hline & & & & $\mathrm{PtN}_{2} \mathrm{CP}$ & $\begin{array}{c}\mu \mathrm{N} \\
\mathrm{MeC} \\
\mathrm{Ph}_{3} \mathrm{P}\end{array}$ & $\begin{array}{l}2.14(1,0) \\
2.08(1) \\
2.204(2)\end{array}$ & $\begin{array}{l}3.117(1) \\
\text { N 95.1(3) }\end{array}$ & $\begin{array}{l}\mathrm{N}, \mathrm{N} \\
\mathrm{C}, \mathrm{P} \\
\mathrm{N}, \mathrm{C} \\
\\
\mathrm{N}, \mathrm{P}\end{array}$ & $\begin{array}{c}78.2(3) \\
88.9(3) \\
90.3(4) \\
168.5(3) \\
102.5(2)\end{array}$ & \\
\hline $\begin{array}{c}{\left[\mathrm{Pt}\left(\mu-\eta^{2}-\mathrm{COCH}_{2} \mathrm{CH}_{2} \mathrm{~N}\right) .\right.} \\
\left.(\mathrm{Cl})\left(\mathrm{PPh}_{3}\right)_{2}\right]_{2} . \\
0.5 \mathrm{ClCH}_{2} \mathrm{CH}_{2} \mathrm{Cl} \\
\text { (yellow) }\end{array}$ & $\begin{array}{c}\operatorname{tr} \\
\mathrm{P}-1 \\
4\end{array}$ & $\begin{array}{l}19.564(3) \\
16.497(3) \\
13.243(2)\end{array}$ & $\begin{array}{l}88.93(3) \\
97.88(3) \\
91.97(3)\end{array}$ & PtNCClP & $\begin{array}{c}\mu \eta^{2} \mathrm{~N} \\
\mu \eta^{2} \mathrm{C} \\
\mathrm{Cl} \\
\mathrm{Ph}_{3} \mathrm{P}\end{array}$ & $\begin{array}{c}2.07(2,0) \\
1.95(2,2) \\
2.452(4,11) \\
2.239(6,6)\end{array}$ & $3.407(1)$ & $\begin{array}{c}\mathrm{N}, \mathrm{C} \\
\mathrm{Cl}, \mathrm{P} \\
\mathrm{N}, \mathrm{Cl} \\
\mathrm{N}, \mathrm{P} \\
\mathrm{C}, \mathrm{Cl} \\
\mathrm{C}, \mathrm{P}\end{array}$ & $\begin{array}{c}85.8(7,1) \\
91.2(2,3) \\
91.0(2,5) \\
174.8(5,3) \\
174.5(6,1.4) \\
93.7(6,8)\end{array}$ & [79] \\
\hline \multirow[t]{2}{*}{$\begin{array}{c}\mathrm{Pt}_{2}\left(\mu-\eta^{2}-\mathrm{dmpm}\right)_{2}(\mathrm{Me})_{4} \\
(\text { colourless })\end{array}$} & $\begin{array}{c}\mathrm{m} \\
\mathrm{P} 2{ }_{1} / \mathrm{a} \\
4\end{array}$ & $\begin{array}{l}16.634(4) \\
11.112(3) \\
12.469(3)\end{array}$ & $103.40(2)$ & $\mathrm{PtC}_{2} \mathrm{P}_{2}$ & $\begin{array}{l}\mu \eta^{2} \mathrm{P} \\
\mathrm{MeC}\end{array}$ & $\begin{array}{c}2.270(4,6) \\
2.168(12,13)\end{array}$ & $4.198(1)$ & $\begin{array}{l}\mathrm{P}, \mathrm{P} \\
\mathrm{C}, \mathrm{C} \\
\mathrm{P}, \mathrm{C}\end{array}$ & $\begin{array}{c}102.7(2) \\
82.8(5) \\
87.5(4,2) \\
169.7(4,2)\end{array}$ & [80] \\
\hline & & & & $\mathrm{PtC}_{2} \mathrm{P}_{2}$ & $\begin{array}{l}\mu \eta^{2} \mathrm{P} \\
\mathrm{MeC}\end{array}$ & $\begin{array}{c}2.280(3,0) \\
2.142(12,18)\end{array}$ & $4.276(1)$ & $\begin{array}{l}\mathrm{P}, \mathrm{P} \\
\mathrm{C}, \mathrm{C} \\
\mathrm{P}, \mathrm{C}\end{array}$ & $\begin{array}{c}102.9(2) \\
83.4(5) \\
86.9(4,4) \\
170.3(4,3)\end{array}$ & \\
\hline $\begin{array}{c}{\left[\mathrm{Pt}_{2}(\mu-\mathrm{H})(\mathrm{Ph})_{2}\left(\mathrm{Pme}_{3}\right)_{4}\right]} \\
\mathrm{Bph}_{4} \\
(\text { white })\end{array}$ & & & & $\mathrm{PtP}_{2} \mathrm{HC}$ & $\begin{array}{c}\mu \mathrm{H} \\
\mathrm{Me}_{3} \mathrm{P} \\
\mathrm{PhC}\end{array}$ & $\begin{array}{c}\text { not given } \\
2.286(4,5) \\
2.04(4,2)\end{array}$ & $\begin{array}{c}3.0969(9) \\
\text { H not given }\end{array}$ & $\begin{array}{l}\mathrm{P}, \mathrm{P} \\
\mathrm{P}, \mathrm{C}\end{array}$ & $\begin{array}{c}172.0(2,4) \\
87.2(4,6) \\
166.0(5,1.0)\end{array}$ & \\
\hline \multirow[t]{2}{*}{$\begin{array}{c}\mathrm{Pt}_{2}(\mu-\eta: \eta \text {-btmset }) . \\
\left(\mathrm{Pet}_{3}\right)_{4}(\mathrm{Ph})_{2} \\
\text { (pale yellow) }\end{array}$} & $\begin{array}{c}\operatorname{tr} \\
\mathrm{P}-1 \\
3\end{array}$ & $\begin{array}{c}16.804(3) \\
25.107(5) \\
9.221(2)\end{array}$ & $\begin{array}{l}98.40(3) \\
99.67(3) \\
88.12(3)\end{array}$ & $\mathrm{PtC}_{2} \mathrm{P}_{2}$ & $\begin{array}{l}\mu \eta \mathrm{C} \\
\mathrm{PhC} \\
\mathrm{Et}_{3} \mathrm{P}\end{array}$ & $\begin{array}{c}2.03(2,1) \\
2.09(1,1) \\
2.286(6,12)\end{array}$ & & $\begin{array}{l}\mathrm{C}, \mathrm{C} \\
\mathrm{P}, \mathrm{P} \\
\mathrm{C}, \mathrm{P}\end{array}$ & $\begin{array}{c}176(2,2) \\
176.4(2,7) \\
89.1(6,2.4)\end{array}$ & [82] \\
\hline & & & & $\mathrm{PtC}_{2} \mathrm{P}_{2}$ & $\begin{array}{l}\mu \eta \mathrm{C} \\
\mathrm{PhC} \\
\mathrm{Et}_{3} \mathrm{P}\end{array}$ & $\begin{array}{c}2.03(2) \\
2.09(2) \\
2.2985,5)\end{array}$ & & $\begin{array}{l}\mathrm{C}, \mathrm{C} \\
\mathrm{P}, \mathrm{P} \\
\mathrm{C}, \mathrm{P}\end{array}$ & $\begin{array}{c}179.0(7) \\
176.1(2) \\
89.3(5,2.1)\end{array}$ & \\
\hline \multirow[t]{2}{*}{$\begin{array}{c}\mathrm{Pt}_{2}(\mu-\eta: \eta-b t m s e b t) \\
\left(\mathrm{Pet}_{3}\right)_{4}(\mathrm{Ph})_{2} \\
(\text { yellow })\end{array}$} & $\begin{array}{c}\operatorname{tr} \\
\mathrm{P}-1 \\
2\end{array}$ & $\begin{array}{c}15.870(6) \\
17.460(8) \\
9.525(4)\end{array}$ & $\begin{array}{l}97.28(3) \\
90.80(3) \\
82.06(3)\end{array}$ & $\mathrm{PtC}_{2} \mathrm{P}_{2}$ & $\begin{array}{l}\mu \eta \mathrm{C} \\
\mathrm{PhC} \\
\mathrm{Et}_{3} \mathrm{P}\end{array}$ & $\begin{array}{c}1.96(2) \\
2.03(2) \\
2.312(6,5)\end{array}$ & & $\begin{array}{l}\mathrm{C}, \mathrm{C} \\
\mathrm{P}, \mathrm{P} \\
\mathrm{C}, \mathrm{P}\end{array}$ & $\begin{array}{c}179.0(8) \\
178.2(2) \\
90.2(6,2)\end{array}$ & [82] \\
\hline & & & & $\mathrm{PtC}_{2} \mathrm{P}_{2}$ & $\begin{array}{l}\mu \eta \mathrm{C} \\
\mathrm{PhC} \\
\mathrm{Et}_{3} \mathrm{P}\end{array}$ & $\begin{array}{c}2.05(2) \\
2.06(2) \\
2.293(6,3)\end{array}$ & & $\begin{array}{l}\mathrm{C}, \mathrm{C} \\
\mathrm{P}, \mathrm{P} \\
\mathrm{C}, \mathrm{P}\end{array}$ & $\begin{array}{c}176.8(7) \\
174.5(2) \\
89.9(5,2.4)\end{array}$ & \\
\hline
\end{tabular}




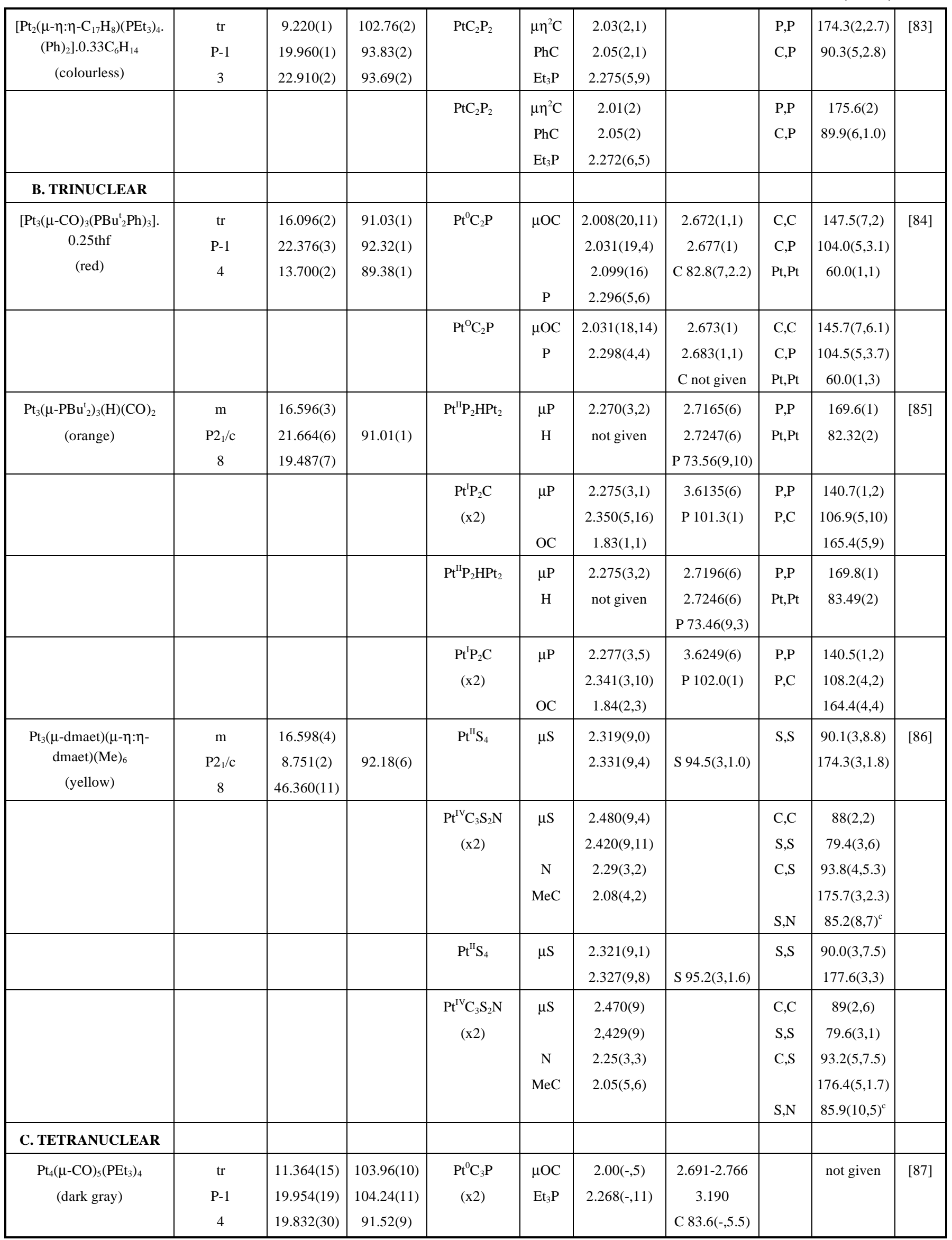


(Table 3).contd.....

\begin{tabular}{|c|c|c|c|c|c|c|c|c|c|c|}
\hline & & & & $\begin{array}{l}\mathrm{Pt}^{0} \mathrm{C}_{2} \mathrm{P} \\
(\mathrm{x} 2)\end{array}$ & $\begin{array}{l}\mu \mathrm{OC} \\
\mathrm{Et}_{3} \mathrm{P}\end{array}$ & $\begin{array}{c}2.06(-, 2) \\
2.24 \\
2.228(-, 6)\end{array}$ & & & & \\
\hline $\begin{array}{c}{\left[\mathrm{Pt}_{4}\left(\mu_{3}-\mathrm{I}\right)_{4}\left(\mathrm{Me}_{12}\right] .0 .5 \mathrm{MeI}\right.} \\
\text { (colourless) }\end{array}$ & $\begin{array}{l}\mathrm{rh} \\
\mathrm{R} 3 \\
12\end{array}$ & $\begin{array}{l}10.3139(4) \\
90.555(8)\end{array}$ & & $\begin{array}{c}\mathrm{Pt}^{\mathrm{IV}} \mathrm{C}_{3} \mathrm{I}_{3} \\
(\mathrm{x} 4)\end{array}$ & $\mu_{3} \mathrm{I}$ & $\begin{array}{c}2.8105(8) \\
2.8328(8,38) \\
2.059(13,14)\end{array}$ & $\begin{array}{c}4.105(1) \\
\text { I } 93.0(1,5)\end{array}$ & $\begin{array}{c}\mathrm{C}, \mathrm{C} \\
\mathrm{I}, \mathrm{I} \\
\mathrm{C}, \mathrm{I}\end{array}$ & $\begin{array}{c}88.4(6,1.0) \\
86.7(1,4) \\
92.5(4,3.0) \\
177.5(4,1.1)\end{array}$ & [88] \\
\hline \multirow[t]{2}{*}{$\mathrm{Pt}_{4}(\mu-\mathrm{Cl})_{4}\left(\mu-\eta^{3} \text {-allyl }\right)_{4}$} & $\begin{array}{c}\operatorname{tr} \\
\mathrm{P}-1 \\
4\end{array}$ & $\begin{array}{l}13.34(3) \\
16.16(3) \\
8.33(1)\end{array}$ & $\begin{array}{l}90.2(2) \\
100.2(2) \\
93.2(2)\end{array}$ & $\begin{array}{c}\mathrm{PtC}_{3} \mathrm{Cl}_{3} \\
(\mathrm{x} 4)\end{array}$ & $\begin{array}{l}\mu \mathrm{Cl} \\
\eta^{3} \mathrm{C}\end{array}$ & $\begin{array}{c}2.37(2,1) \\
2.49(2,3) \\
1.98(8,11) \\
2.20(12,7)\end{array}$ & $\begin{array}{c}3.236(4,12) \\
3.278(4,5) \\
\text { Cl } 83.7(6,1.2)\end{array}$ & $\mathrm{Cl}, \mathrm{Cl}$ & $85.0(7,7)$ & [89] \\
\hline & & & & $\begin{array}{c}\mathrm{PtC}_{3} \mathrm{Cl}_{3} \\
(\mathrm{x} 4)\end{array}$ & $\begin{array}{l}\mu \mathrm{Cl} \\
\eta^{3} \mathrm{C}\end{array}$ & $\begin{array}{l}2.37(2,3) \\
2.49(2,2) \\
2.08(9,5) \\
2.20(9,5)\end{array}$ & $\begin{array}{c}3.233(4,3) \\
3.262(4,0) \\
\mathrm{Cl} 83.6(7,8)\end{array}$ & $\mathrm{Cl}, \mathrm{Cl}$ & $85.0(7,5)$ & \\
\hline
\end{tabular}

Footnotes: a). Where more than one chemically equivalent distance or angle is present, the mean value is tabulated. The first number in parenthesis is the e.s.d., and the second is the maximum deviation from the mean.

b). The chemical identity of the coordinated atom or ligand is specified in these columns

c). Five-membered metallocyclic ring.

are three methyl groups in the cis configuration, two oxygen atom forming a chelate salicylaldehyde, and one bridging oxygen atom $\left(\mathrm{PtO}_{3} \mathrm{C}_{3}\right)$. The mean $\mathrm{Pt}-\mathrm{O}$ - Pt bridge angle and Pt...Pt separation are $100(1)^{\circ}$ and 3.407(4) $\AA$ (molecule 1) and $102(1)^{\circ}$ and $3.418(4) \AA$ (molecule 2$)$.

The asymmetric unit of colourless $\left[\mathrm{Pt}_{2}(\mu-\right.$ $\left.\mathrm{NH}_{2}\right)_{2}(\mathrm{Me})_{2}\left(\mathrm{PPh}_{3}\right)_{2}$ ] [78] consists of two independent but chemically identical half molecules on sites with a common 2-fold rotation axis. Two $\left\{\mathrm{Pt}(\mathrm{Me})\left(\mathrm{PPh}_{3}\right)\right\}^{+}$fragments are connected by two $\mathrm{NH}_{2}$ groups, which serve as bridges and form a four - membered centrosymmetrical $\mathrm{Pt}(\mathrm{N})_{2} \mathrm{Pt}$ ring, with the mean $\mathrm{Pt}-\mathrm{N}$ - Pt bond angles of 95.2 and $95.1^{\circ}$ and $\mathrm{Pt}(\mathrm{II})$... Pt(II) separations of 3.106(1) and 3.117(1) $\AA$, respectively.

The structure of one of the two independent molecules of yellow $\left[\mathrm{Pt}\left(\mu-\eta^{2}-\mathrm{COCH}_{2} \mathrm{CH}_{2} \mathrm{~N}\right)(\mathrm{Cl})\left(\mathrm{PPh}_{3}\right)\right]_{2}$ [79] is shown in (Fig. 3). Each dimer is formed by two bridging $\mathrm{COCH}_{2} \mathrm{CH}_{2} \mathrm{~N}$ ligands which are coordinated by one $\mathrm{Pt}(\mathrm{II})$ on one side via a $\mathrm{Pt}-\mathrm{N}$ bond and to the second $\mathrm{Pt}(\mathrm{II})$ via $\mathrm{Pt}-\mathrm{C}$ bond forming a six membered $(-\mathrm{Pt}-\mathrm{C}-\mathrm{N}-) 2$ ring . The $\mathrm{Pt}(\mathrm{II})$...Pt(II) separations are 3.407(1) and 3.420(1) $\AA$. The coordination geometry around each $\mathrm{Pt}(\mathrm{II})$ atom is an irregular square with deviation in the tetrahedral direction of the bonded atoms (PtNCClP).

Structure of colourless $\mathrm{Pt}_{2}\left(\mu-\eta^{2}-\mathrm{dmpm}\right)_{2}(\mathrm{Me})_{4}$ [80] contains the eight membered $\{\mathrm{PtPCP}-\}_{2}$ ring with $\mathrm{Pt}(\mathrm{II}) \ldots \mathrm{Pt}(\mathrm{II})$ separations of 4.198(1) $\AA$ (molecule 1) and 4.276(1) (molecule 2). The ring adopts the twist - sadl conformation. Each $\mathrm{Pt}(\mathrm{II})$ atom displays a cis - square - planar coordination $\left(\mathrm{PtC}_{2} \mathrm{P}_{2}\right)$.

In white $\left[\mathrm{Pt}_{2}(\mu-\mathrm{H})(\mathrm{Ph})_{2}\left(\mathrm{PMe}_{3}\right)_{4}\right] \mathrm{BPh}_{4}$ [81] both complex cations contain the two planar $\left\{\mathrm{Pt}(\mathrm{Ph})_{2}\left(\mathrm{PMe}_{3}\right)_{2}\right\}$ moieties bridged by a single hydride ligand. The Pt(II) ... Pt(II) distances are 3.061(7 and 3.0969(9) $\AA$. The $\mathrm{Pt}-\mathrm{H}-\mathrm{Pt}$ bridge angle is $121(5)^{\circ}$.

There are two Pt(II) dimers: pale yellow and yellow [82] and both contain two independent dimer molecules. In each case, the crystal structure consists of discrete dimeric molecules. A pair of $\mathrm{Pt}(\mathrm{Ph})\left(\mathrm{PEt}_{3}\right)_{2}$ fragments are bridged by a thiophenediyl or bis- (acetylide) ligand for the pale yellow and yellow, respectively. Each $\mathrm{Pt}(\mathrm{II})$ atom has a trans square - planar geometry $\left(\mathrm{PtC}_{2} \mathrm{P}_{2}\right)$.

In colourless $\left[\mathrm{Pt}_{2}\left(\mu-\eta: \eta-\mathrm{C}_{17} \mathrm{H}_{8}\right)\left(\mathrm{PEt}_{3}\right)_{4}(\mathrm{Ph})_{2}\right] .0 .33 \mathrm{C}_{6} \mathrm{H}_{14}$ [83] two $\mathrm{Pt}(\mathrm{Ph})\left(\mathrm{PEt}_{3}\right)_{2}$ fragment are bridged by 2,7- 
bis(ethynyl) fluorene. Each $\mathrm{Pt}(\mathrm{II})$ has a trans -square planar geometry $\left(\mathrm{PtC}_{2} \mathrm{P}_{2}\right)$.

There are three trimer derivatives each containing two independent trimeric molecules (Table 3B). In red $\left[\mathrm{Pt}_{3}(\mu-\right.$ $\mathrm{CO})_{3}\left(\mathrm{PBu}_{2}^{\mathrm{t}} \mathrm{Ph}\right)_{3}$ ]. 0.25thf [84] each molecule contains a triangular core of $\mathrm{Pt}_{3}$ atoms. The angle between the $\mathrm{Pt}_{3}$ planes in the two different molecules is $95.0^{\circ}$. The $\mathrm{Pt}-\mathrm{Pt}$ distances span a range 2.672(1) to 2.677(1) $\AA$ in one and 2.673(1) to 2.684(1) in another molecule. Each Pt - Pt edge is bridged by $\mathrm{CO}$ group and $\mathrm{PBu}_{2}^{\mathrm{t}} \mathrm{Ph}$ ligand is attached to each $\mathrm{Pt}(0)$ atom.

The structure of orange $\mathrm{Pt}_{3}\left(\mu-\mathrm{PBu}_{2}^{\mathrm{t}}\right)_{3}(\mathrm{H})(\mathrm{CO})_{2}$ [85] contains an "open" triangular $\mathrm{Pt}_{3}$ units with two short $\mathrm{Pt}(\mathrm{I})-$ $\mathrm{Pt}(\mathrm{II})$ bond distances and a long $\mathrm{Pt}(\mathrm{I})$... Pt(I) separation, each side of this unit is symmetrically bridged by a $\mu-\mathrm{PBu}_{2}{ }_{2}$ group. The mean $\mathrm{Pt}(\mathrm{II})-\operatorname{Pt}(\mathrm{I})$ bond distances and $\mathrm{Pt}(\mathrm{I})$ ...Pt(I) separations are 2.721 and $3.613 \AA$ (molecule 1 ) and 2.722 and $3.625 \AA$ (molecule 2). The Pt(I) - P - Pt(II) and $\mathrm{Pt}(\mathrm{I})-\mathrm{Pt}-\mathrm{P}(1)$ bridge angles are 73.6 (av.) and 101.3(1) ${ }^{\circ}$ (molecule 1) and 73.5 (av.) and $102.0(1)^{\circ}$ (molecule 2), respectively. In this mixed - valence triplatinum (I, I, II), each $\mathrm{Pt}(\mathrm{I})$ atom has tetrahedral $\left(\mathrm{PtP}_{2} \mathrm{CPt}(\mathrm{II})\right)$ and $\mathrm{Pt}(\mathrm{II})$ squareplanar $\left(\mathrm{PtP}_{2} \mathrm{Pt}_{2}(\mathrm{I})\right)$ arrangement.

The structure of one of two independent molecules of $\left[\mathrm{Pt}_{3}(\mathrm{dmaet})_{4}(\mathrm{Me})_{6}\right][86]$ is shown in (Fig. 4). As can be seen, two Pt(IV) $(\mathrm{Me})_{3}\left(\mathrm{SCH}_{2} \mathrm{CH}_{2} \mathrm{NMe}_{2}\right)_{2}$ moieties are connected through the pairs of $\mathrm{S}$ donor atoms which coordinated to the central $\mathrm{Pt}(\mathrm{II})$ atom. Each $\mathrm{Pt}(\mathrm{IV})$ atom shows distorted pseudo octahedral geometry $\left(\mathrm{PtC}_{3} \mathrm{~S}_{2} \mathrm{~N}\right)$. The $\mathrm{Pt}(\mathrm{II})$ atom in each molecule shows distorted square planar geometry $\left(\mathrm{PtS}_{4}\right)(\mathrm{Ta}-$ ble 3B).

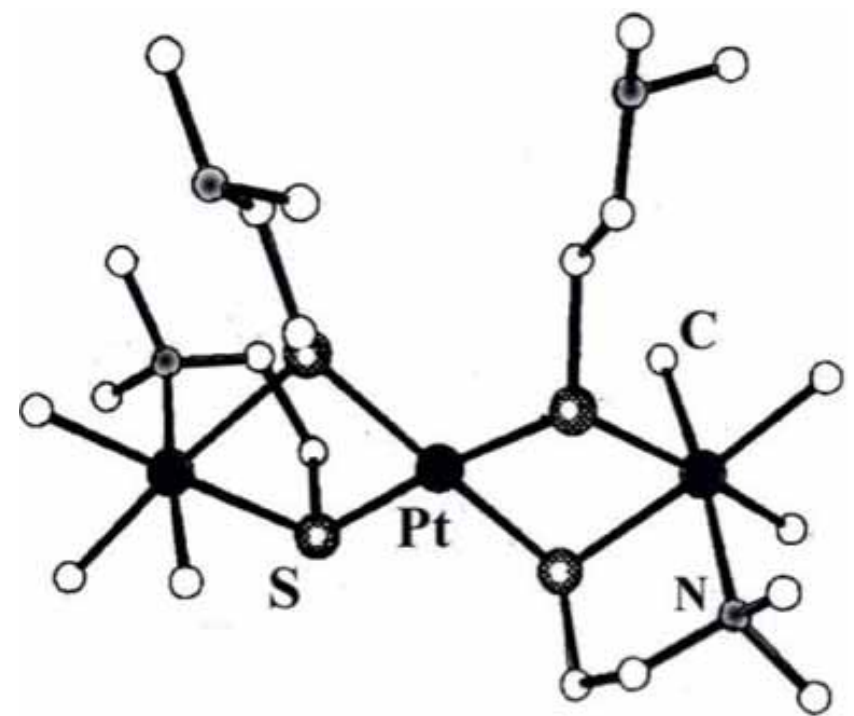

Fig. (4). Structure of one independent molecule of $\left[\mathrm{Pt}_{3}(\mathrm{dmaet})_{4}\right.$ $\left.(\mathrm{Me})_{6}\right]$ [86].

There are also three tetranuclear derivatives, which contain two crystallographically independent molecules, as well (Table 3C). In each molecule of dark gray $\mathrm{Pt}_{4}(\mu-\mathrm{CO})_{5}\left(\mathrm{PEt}_{3}\right)_{4}$ [87], the four $\operatorname{Pt}(0)$ atoms are located at the apices of a deformed tetrahedron (butterfly), in which five edges have PtPt bond lengths in range 2.69-2.766 (av. 2.731 $\AA$ ) and 2.7042.745 (av. $2.727 \AA$ ) (in molecule 1 and 2) respectively while the six edge, are extended to 3.190 and $3.263 \AA$, respec- tively. Bridging $\mathrm{CO}$ groups are bound to each shortened edge of $\mathrm{Pt}_{4}$ tetrahedron. The $\mathrm{PEt}_{3}$ molecules are terminal ligands of the $\operatorname{Pt}(0)$ atom.

The structure of colourless $\left[\mathrm{Pt}_{4}\left(\mu^{3}-\mathrm{I}\right)_{4}(\mathrm{Me})_{12}\right] .0 .5 \mathrm{MeI}$ [88] is shown in (Fig. 5). Each cluster has $\mathrm{C}_{3}$ symmetry. A distorted "cubane skeleton" was found for the cluster, with octahedrally coordinated $\mathrm{Pt}(\mathrm{IV})$ atom $\left(\mathrm{PtC}_{3} \mathrm{I}_{3}\right)$ and tetrahedrally coordinated iodine atoms. The sum of six $\mathrm{Pt}-\mathrm{L}$ bond distances in molecule 1 is $15.404 \AA$ which is about $0.036 \AA$ smaller than that found in molecule 2 , which means that the former is less crowded than the latter. The mean Pt...Pt separation is 4.105(1) $\AA$ and mean $\mathrm{Pt}$ - I - Pt bridge angle $93.0^{\circ}$.

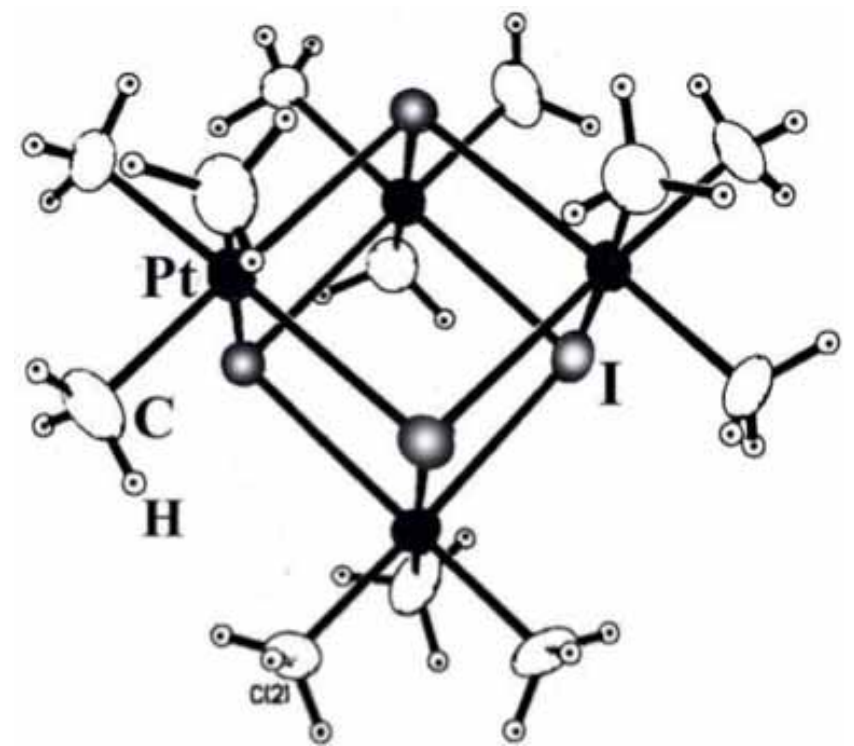

Fig. (5). Structure of one independent molecule of $\left[\mathrm{Pt}_{4}\left(\mu_{3}-\right.\right.$ $\left.\mathrm{I})_{4}(\mathrm{Me})_{12}\right] .0 .5 \mathrm{MeI}[88]$.

Inspection of the data in Tables $\mathbf{1 - 3}$ reveals that there are over ninety platinum distortion isomers, which can be divided into several subgroups. In the series of mononuclear derivatives one example, $\mathrm{Pt}(\mathrm{II})\left(\mathrm{CH}_{2} \mathrm{Cl}\right)\left(\eta_{3}-\mathrm{tmba}\right) \mathrm{Cl}$ [37] exists in three isomeric forms. Nineteen exist in two isomeric forms $(\mathrm{Pt}(0)$ [7], $\mathrm{Pt}(\mathrm{II})$ [8-34], $\mathrm{Pt}(\mathrm{IV})[35,36])$. One example $\mathrm{Pt}(\mathrm{II})\left(\mathrm{SMe}_{2}\right)_{2}(\mathrm{Ph})(\mathrm{Cl})$ [12] exists in two isomeric forms (triclinic and monoclinic) and monoclinic contains two crystallographically independent molecules.

There are thirty six examples $(\operatorname{Pt}(0)$ [38-42], $\mathrm{Pt}(\mathrm{II})$ [12, 37, 42-65], Pt(IV) [66-71]) which contains two crystallographicaly independent molecules. One example, $\left[\mathrm{Pt}(\mathrm{II})\left(\eta^{4}-\mathrm{bdpb}\right) \mathrm{Cl}\right] \mathrm{Cl}[72]$ contains three such molecules and another one $\left[\mathrm{Pt}(\mathrm{II})\left(\eta^{2}\right.\right.$-dmaen $)\left(\eta^{2}\right.$-msedpa $\left.)\right] . \mathrm{ClO}_{4}$ [73] contains even four such molecules.

$\mathrm{Bi}-$, tri- and tetranuclear derivatives are found only derivatives, which contain two crystallographically independent molecules. The platinum in these derivatives are found in the oxidation states of zero [84, 87], +1 [74], +2 [75, 76, 7983, 89], and mixed valence $\mathrm{Pt}(\mathrm{I})(\mathrm{x} 2)$ plus $\mathrm{Pt}(\mathrm{II})$ [85] and Pt(II) plus Pt(IV) (x2) [86].

In this series of distortion isomers the $\mathrm{Pt}(0)$ atoms are tetrahedrally coordinated $\left(\mathrm{PtP}_{3} \mathrm{C}[7,8], \mathrm{PtC}_{4}[38], \mathrm{PtC}_{2} \mathrm{P}_{2}\right.$ [39-42], $\left.\mathrm{PtC}_{2} \mathrm{PPt}^{\prime}[84,87]\right)$. The mean $\mathrm{Pt}(0)-\mathrm{L}$ bond distances elongated in the order: $1.86 \AA(\mathrm{CO})<2.055 \AA(\mu-\mathrm{CO})$ 
$<2.066 \AA$ (bi-CL) $<2.273 \AA$ (bi-PL) < $2.278 \AA$ (PL). The $\mathrm{Pt}(0)-\operatorname{Pt}(0)$ bond distances range from 2.671(1) - 2.766(1) $\AA$ (av. $2.709 \AA$ ).
The $\mathrm{Pt}(\mathrm{I})$ is tetrahedrally coordinated (PtCSPPt') [74] with the mean $\mathrm{Pt}(1)-\mathrm{L}$ bond distances elongated in the order: $1.845 \AA(\mathrm{CO})<2.263 \AA(\mu-\mathrm{S})<2.267 \AA(\mu-\mathrm{PL})<$

Table 4. Crystallographic and Structural Data for Platinum Organometallic Complexes - Cis- and Trans- Isomers ${ }^{\mathrm{a}}$

\begin{tabular}{|c|c|c|c|c|c|c|c|c|c|}
\hline $\begin{array}{l}\text { COMPOUND } \\
\text { (colour) }\end{array}$ & $\begin{array}{c}\text { Cryst. cl. } \\
\text { SPACE GR. } \\
\text { Z }\end{array}$ & $\begin{array}{l}\mathbf{a}[\AA ̊ \AA] \\
\mathbf{b}[\AA ̊ \\
\mathrm{c}[\AA]\end{array}$ & $\begin{array}{l}\alpha\left[^{\circ}\right] \\
\beta\left[^{\circ}\right] \\
\gamma\left[^{\circ}\right]\end{array}$ & $\begin{array}{l}\text { Chromo- } \\
\text { PHORE }\end{array}$ & & $\begin{array}{l}t-\mathbf{L} \\
{[\AA ̊}\end{array}$ & & $\begin{array}{l}\mathbf{P t}-\mathbf{L} \\
{\left[{ }^{\circ}\right]}\end{array}$ & Ref. \\
\hline $\begin{array}{c}\text { cis- }\left[\mathrm{Pt}(\mathrm{ozl})_{2}(\mathrm{CN})_{2}\right] \cdot \mathrm{H}_{2} \mathrm{O} \\
\text { (orange) }\end{array}$ & $\begin{array}{c}\operatorname{tr} \\
\mathrm{P}-1 \\
2\end{array}$ & $\begin{array}{l}8.085(2) \\
8.379(2) \\
8.669(1)\end{array}$ & $\begin{array}{l}78.78(1) \\
71.17(1) \\
79.09(1)\end{array}$ & $\mathrm{PtC}_{4}$ & $\begin{array}{l}\mathrm{NC}^{\mathrm{b}} \\
\mathrm{ozlC}\end{array}$ & $\begin{array}{l}1.997(8,7) \\
2.015(7,5)\end{array}$ & $\mathrm{C}, \mathrm{C}^{\mathrm{b}}$ & $\begin{array}{l}90.0(3,4.7) \\
176.1(3,1.2)\end{array}$ & [90] \\
\hline $\begin{array}{l}\text { cis- } \mathrm{Pt}\left\{\mathrm{CH}_{2}=\mathrm{C}(\mathrm{Me})\right\} \\
\left(\mathrm{C} \equiv \mathrm{CBu}^{\mathrm{t}}\right)\left(\mathrm{PPh}_{3}\right)_{2} \\
(\text { colourless })\end{array}$ & $\begin{array}{c}\mathrm{m} \\
\mathrm{P}_{2} / \mathrm{n} \\
4\end{array}$ & $\begin{array}{l}14.000(3) \\
11.991(4) \\
22.602(4)\end{array}$ & $90.50(1)$ & $\mathrm{PtC}_{2} \mathrm{P}_{2}$ & $\begin{array}{l}\mathrm{C} \\
\mathrm{P}\end{array}$ & $\begin{array}{c}2.05(2) \\
1.97(2) \\
2.340(4) \\
2.286(4)\end{array}$ & $\begin{array}{l}\mathrm{C}, \mathrm{C} \\
\mathrm{P}, \mathrm{P} \\
\mathrm{C}, \mathrm{P}\end{array}$ & $\begin{array}{c}84.3(7) \\
100.3(1) \\
87.7(5,4.6) \\
171.9(4,4.7)\end{array}$ & [91] \\
\hline $\begin{array}{c}\text { cis- }\left[\mathrm{Pt}(\mathrm{COEt})(\mathrm{COPh})\left(\mathrm{PPh}_{3}\right)_{2}\right] . \\
0.5 \mathrm{C}_{6} \mathrm{H}_{6} \\
(\text { white })\end{array}$ & $\begin{array}{c}\mathrm{m} \\
\mathrm{P} 2 / \mathrm{c} \\
4\end{array}$ & $\begin{array}{l}17.770(8) \\
10.537(1) \\
23.739(9)\end{array}$ & 111.52(4) & $\mathrm{PtC}_{2} \mathrm{P}_{2}$ & $\begin{array}{l}\mathrm{C} \\
\mathrm{C} \\
\mathrm{P}\end{array}$ & $\begin{array}{c}2.00(2) \\
1.98(3) \\
2.329(6,0)\end{array}$ & $\begin{array}{l}\mathrm{C}, \mathrm{C} \\
\mathrm{P}, \mathrm{P} \\
\mathrm{C}, \mathrm{P}\end{array}$ & $\begin{array}{c}81.6(9) \\
100.5(2) \\
88.9(7,2) \\
169.0(7,1.1)\end{array}$ & [92] \\
\hline $\begin{array}{c}\text { trans- }[\mathrm{Pt}(\mathrm{COEt})(\mathrm{COPh}) \\
\left.\left(\mathrm{PPh}_{3}\right)_{2}\right] \cdot 0.5 \mathrm{C}_{6} \mathrm{H}_{6} \\
\text { (white) }\end{array}$ & $\begin{array}{c}\mathrm{m} \\
\mathrm{P} 2 / \mathrm{n} \\
4\end{array}$ & $\begin{array}{l}12.204(8) \\
14.542(8) \\
24.659(4)\end{array}$ & $102.10(4)$ & $\mathrm{PtC}_{2} \mathrm{P}_{2}$ & $\begin{array}{l}\mathrm{C} \\
\mathrm{C} \\
\mathrm{P}\end{array}$ & $\begin{array}{c}2.04(2) \\
2.01(2) \\
2.306(6,5)\end{array}$ & $\begin{array}{l}\mathrm{C}, \mathrm{C} \\
\mathrm{P}, \mathrm{P} \\
\mathrm{C}, \mathrm{P}\end{array}$ & $\begin{array}{c}176.0(8) \\
175.8(3) \\
90.0(6,3.0)\end{array}$ & [92] \\
\hline $\begin{array}{c}\text { cis- } \mathrm{PtCl}_{2}\left\{\eta-\mathrm{C}\left(\mathrm{PhNCH}_{2}\right)_{2}\right\} .\left(\mathrm{PEt}_{3}\right) \\
\text { (colourless) }\end{array}$ & $\begin{array}{c}\text { or } \\
\text { Pnam } \\
4\end{array}$ & $\begin{array}{l}15.991(2) \\
8.601(2) \\
16.703(2)\end{array}$ & & $\mathrm{PtCl}_{2} \mathrm{CP}$ & $\begin{array}{c}\mathrm{Cl} \\
\eta \mathrm{C} \\
\mathrm{Et}_{3} \mathrm{P}\end{array}$ & $\begin{array}{c}2.362(3) \\
2.381(3) \\
2.009(13) \\
2.234(3)\end{array}$ & $\begin{array}{c}\mathrm{Cl}, \mathrm{Cl} \\
\mathrm{C}, \mathrm{P} \\
\mathrm{Cl}, \mathrm{C} \\
\\
\mathrm{Cl}, \mathrm{P}\end{array}$ & $\begin{array}{c}89.3(1) \\
93.7(4) \\
91.5(4) \\
179.8(4) \\
86.6(1) \\
174.9(1)\end{array}$ & [93] \\
\hline $\begin{array}{c}\text { cis-[Pt }\left(\mathrm{PPh}_{3}\right)_{2}\left\{\eta-\mathrm{CH}_{2} \mathrm{P}(\mathrm{O}) .\right. \\
\left.\left.(\mathrm{OMe})_{2}\right\} \mathrm{I}\right] \cdot \mathrm{H}_{2} \mathrm{O} \\
\text { (pale yellow) }\end{array}$ & $\begin{array}{c}\mathrm{m} \\
\mathrm{P} 2 / \mathrm{n} \\
4\end{array}$ & $\begin{array}{l}16.981(3) \\
12.870(3) \\
17.309(3)\end{array}$ & 96.71(1) & $\mathrm{PtP}_{2} \mathrm{CI}$ & $\begin{array}{c}\mathrm{Ph}_{3} \mathrm{P} \\
\eta \mathrm{C} \\
\mathrm{I}\end{array}$ & $\begin{array}{c}2.257(2) \\
3.331(2) \\
2.124(6) \\
2.6561(7)\end{array}$ & $\begin{array}{l}\mathrm{P}, \mathrm{P} \\
\mathrm{C}, \mathrm{I} \\
\mathrm{P}, \mathrm{C} \\
\\
\mathrm{P}, \mathrm{I}\end{array}$ & $\begin{array}{c}97.97(2) \\
85.4(2) \\
99.8(4) \\
169.9(2) \\
85.98(5) \\
171.56(5)\end{array}$ & [94] \\
\hline $\begin{array}{c}\operatorname{trans}-\left[\mathrm{Pt}\left(\mathrm{PPh}_{3}\right)_{2}\left\{\eta-\mathrm{CH}_{2} \mathrm{P}(\mathrm{O})\right.\right. \\
\left.\left.(\mathrm{OMe})_{2}\right\} \mathrm{I}\right] \cdot \mathrm{Me}_{2} \mathrm{CO} \\
\text { (pale yellow) }\end{array}$ & $\begin{array}{c}\mathrm{m} \\
\mathrm{P} 2 / \mathrm{n} \\
4\end{array}$ & $\begin{array}{l}11.678(2) \\
19.664(4) \\
18.788(4)\end{array}$ & $107.36(1)$ & $\mathrm{PtP}_{2} \mathrm{CI}$ & $\begin{array}{c}\mathrm{Ph}_{3} \mathrm{P} \\
\eta \mathrm{C} \\
\mathrm{I}\end{array}$ & $\begin{array}{l}2.309(2,4) \\
2.080(8) \\
2.6774(8)\end{array}$ & $\begin{array}{r}\mathrm{P}, \mathrm{P} \\
\mathrm{C}, \mathrm{I} \\
\mathrm{P}, \mathrm{C} \\
\mathrm{P}, \mathrm{I}\end{array}$ & $\begin{array}{c}170.69(8) \\
169.9(2) \\
92.4(2,1.0) \\
88.9(1,1.0)\end{array}$ & [94] \\
\hline
\end{tabular}

Footnotes: a). Where more than one chemically equivalent distance or angle is present, the mean value is tabulated. The first number in parenthesis is the e.s.d., and the second is the maximum deviation from the mean.

b). The chemical identity of the coordinated atom or ligand is specified in these columns. 
$2.277 \AA\left(\mathrm{PPh}_{3}\right)<2.346 \AA$ ( $\mu$-PL, trans to $\left.\mathrm{CO}\right)$. The $\mathrm{Pt}(\mathrm{I})-$ $\mathrm{Pt}(\mathrm{I})$ bond lengths are 2.5998(9) and 2.6008(8) $\AA$.

In this series of distortion isomers the predominant square - planar configuration about the $\mathrm{Pt}(\mathrm{II})$ atom is cis rather than trans with wide variety of the chromophores and $\pi$ - complexes, as well. The mean value of Pt(II) $-\mathrm{L}$ bond distances (mutually trans) increases in the orders: $2.03 \AA$ (L $=\mathrm{C})<2.07 \AA(\mathrm{O}$ or $\mathrm{N})<2: 29 \AA(\mathrm{P})<2.30 \AA(\mathrm{Cl})<2.64 \AA$ (I) (monodentate ligands); $2.13 \AA$ (N-unsaturated ligands) $<$ $2.14 \AA(\mathrm{C})<2.25 \AA(\mathrm{N}$-saturated ligands $)<2.26 \AA(\mathrm{P})$ (bidentate ligands). The mean value of $\mathrm{Pt}(\mathrm{II})-\mathrm{L}$ elongated in the order : $2.02 \AA<$ (uni-) $<2.14 \AA$ (bi- or tri-) $<2.21 \AA$ (tetracoordinated). The trans effect on $\mathrm{Pt}(\mathrm{II})-\mathrm{L}$ bond distances (trans to $\mathrm{C}$ ), the $\mathrm{C}$ donor atom increases the lengths: $2.33 \AA$ $(\mathrm{L}=\mathrm{P}$; trans to $\mathrm{CO})<2.38 \AA(\mathrm{Cl})<2.51 \AA(\mathrm{Br})$. The mean value of $\operatorname{Pt}(\mathrm{II})-\mathrm{L}$ (bridge) bond distance elongated in the order: $1.76 \AA(\mu-\mathrm{H})<1.98 \AA(\mu-\mathrm{CL})<2.135 \AA(\mu-\mathrm{NL})<$ $2.26 \AA(\mu-\mathrm{PL})<2.325 \AA(\mu-\mathrm{SL})<2.43 \AA(\mu-\mathrm{Cl})$.

The $\mathrm{Pt}(\mathrm{IV})$ atoms are six coordinated with the chromophores: $\mathrm{PtN}_{2} \mathrm{C}_{2} \mathrm{Se}_{2}$ [35, 36], $\mathrm{PtN}_{3} \mathrm{C}_{3}$ [66], $\mathrm{PtC}_{3} \mathrm{~N}_{2} \mathrm{X}$ (X = I [67, 69] or $\mathrm{Br}[68])), \mathrm{PtN}_{2} \mathrm{C}_{2} \mathrm{Cl}_{2}$ [70], $\mathrm{PtC}_{3} \mathrm{NPI}$ [71], $\mathrm{PtO}_{3} \mathrm{C}_{3}$ [77], $\mathrm{PtC}_{3} \mathrm{~S}_{2} \mathrm{~N}$ [86] and $\mathrm{PtC}_{3} \mathrm{I}_{3}$ [88].

The mean value of $\operatorname{Pt}(\mathrm{IV})-\mathrm{L}$ bond length (mutually trans) increases in the orders: $2.07 \AA(\mathrm{L}=\mathrm{C})<2.31 \AA(\mathrm{Cl})<$ $2.49 \AA(\mathrm{Se})<2.615 \AA(\mathrm{I}) ; 2.11 \AA(\mathrm{bi}-\mathrm{CL})<2.15 \AA(\mathrm{bi}-\mathrm{N}$ unsaturated) $<2.26 \AA$ (bi-N-saturated). The mean value of $\mathrm{Pt}(\mathrm{IV})-\mathrm{L}$ bond lengths trans to $\mathrm{C}$ donor atom ligands (Me, $\mathrm{Ph})$ elongated in the order $2.56 \AA(\mathrm{Br})<2.81 \AA$ (I).

The mean $\mathrm{Pt}$ - bonds lengths for the homobidentate ligands, which includes N-, C-, S-, and P-donors, are somewhat shorter than those of the corresponding monodentate ligands except for the $\mathrm{P}$ - donor ligands in which the opposite is seen. There is a variety of hetero - bidentate ligands: $\mathrm{N} / \mathrm{C}, \mathrm{N} / \mathrm{S}, \mathrm{N} / \mathrm{P}, \mathrm{N} / \mathrm{I}, \mathrm{C} / \mathrm{P}, \mathrm{S} / \mathrm{P}$, and S/As donor atoms. The heterotridentate ligands: with $2 \mathrm{~N} / \mathrm{C}, \mathrm{N} / 2 \mathrm{C}, \mathrm{N} / 2 \mathrm{~S}$ and $\mathrm{C} / 2 \mathrm{P}$ donor atoms, and tetradentate with $3 \mathrm{C} / \mathrm{P}$ donor atoms. All of these have mean $\mathrm{Pt}-\mathrm{L}$ bond distances that follow the same trend as the homobidentate ligands. Correspondingly there is a wide variety of metallocyclic rings, and the effect of both
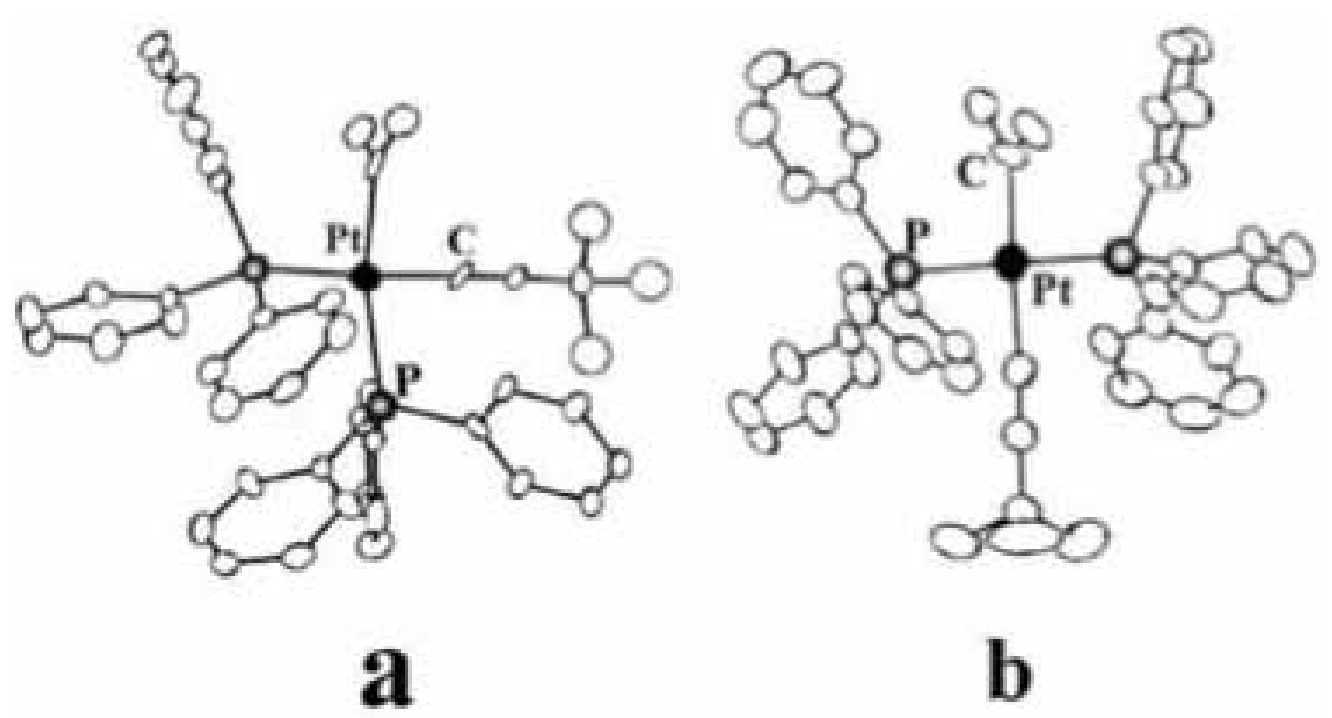
$\left[\mathrm{Pt}\left(\mathrm{H}_{2} \mathrm{C}=\mathrm{CMe}\right)\left(\mathrm{C} \equiv \mathrm{CBu}^{t}\right)\left(\mathrm{PPh}_{3}\right)_{2} \quad[91], \quad[\mathrm{Pt}(\mathrm{COEt})(\mathrm{COPh})\right.$ $\left.\left(\mathrm{PPh}_{3}\right)_{2}\right] \cdot 0.5 \mathrm{C}_{6} \mathrm{H}_{6} \quad[92]$ and $\left[\mathrm{Pt}\left(\mathrm{PPh}_{3}\right)_{2}\left\{\eta-\mathrm{CH}_{2} \mathrm{P}(\mathrm{O})\right.\right.$ $\left.(\mathrm{OMe})_{2} \mathrm{I}\right\} \cdot \mathrm{H}_{2} \mathrm{O}$ [94]. In remaining examples differ from each other not only by configuration but also by crystal class. In $\left[\mathrm{Pt}(\mathrm{ozl})_{2}(\mathrm{CN})_{2}\right] \cdot \mathrm{H}_{2} \mathrm{O}$ [90] cis-isomer is triclinic and transmonoclinic, and in $\left[\mathrm{PtCl}_{2}\left\{\eta-\mathrm{C}\left(\mathrm{PhNCH}_{2}\right)_{2}\right\}\left(\mathrm{PEt}_{3}\right)\right]$ [93] cis- is orthorhombic and trans- triclinic.

A square-planar geometry about each Pt(II) atom is created only by unidentate ligands with the chromophores being: $\mathrm{PtC}_{4}$ [90] $\mathrm{PtC}_{2} \mathrm{P}_{2}$ [91, 92], $\mathrm{PtCl}_{2} \mathrm{CP}$ [93] and $\mathrm{PtC}_{2} \mathrm{CI}$ [94]. The mean values of $\mathrm{Pt}(\mathrm{II})-\mathrm{L}$ bond distances (mutually trans) elongated in the order: $1.973 \AA(\mathrm{CN})<2.024 \AA(\mathrm{CL})$ $<2.302 \AA(\mathrm{Cl})<2.303 \AA\left(\mathrm{PPh}_{3}\right)$. The trans effect on Pt(II) $\mathrm{L}$ bond distances can be divided into two categories. The first in which a hetero-donor atom shortens the trans $\mathrm{Pt}(\mathrm{II})$ $\mathrm{L}$ bond and the second in which the trans donor atom elongates the trans length. As an example are $\mathrm{Pt}(\mathrm{II})-\mathrm{P}\left(\mathrm{PPh}_{3}\right)$ bond distances: $2.257 \AA$ (trans to I) $<2.286 \AA$ (trans to $\mathrm{CH}_{2}=\mathrm{CMe}$ ) $<2.303 \AA$ (mutually trans ) $<2.329 \AA$ (trans to $\mathrm{COX})<2.340 \AA$ (trans to $\mathrm{C} \equiv \mathrm{CBut}$ ). (Table 4). Noteworthy, the cis- L-Pt-L bond angles in the cis-isomers are in the

Fig. (6). Structures of $\mathrm{Pt}\left(\mathrm{CH}_{2}=\mathrm{CMe}\right)\left(\mathrm{C} \equiv \mathrm{CBu}^{\mathrm{t}}\right)\left(\mathrm{PPh}_{3}\right)_{2}$, cis- (a) and trans- (b) [91]. 
range $81.6(9)-100.5(2)^{\circ}$, which is more wide range than that one found in the trans isomer $87.0(2)-93.0(2)^{\circ}$. The deviation of the trans- L-Pt-L bond angles, in cis- derivatives of $11.6^{\circ}$ is somewhat larger than in the trans derivatives of $10^{\circ}$ (range $170-180^{\circ}$ ). This indicates that there are somewhat larger distortions in the cis- isomers than in the transisomers.

\section{LIGAND ISOMERISM}

There are three $\mathrm{Pt}(\mathrm{II})$ complexes (Table 5) which exhibit this type of isomerism: red orange $\mathrm{Pt}\left(\eta^{2}-\mathrm{th}-4,5-\mathrm{ppy}\right)_{2}$ and $\operatorname{Pt}\left(\eta^{2} \text {-th-5,6-ppy }\right)_{2}$ [95]; yellow $\mathrm{PtCl}_{3}\left(\eta^{2}-\mathrm{C}_{4} \mathrm{H}_{10} \mathrm{~N}-2\right)$ [24] and $\mathrm{PtCl}_{3}\left(\eta^{2}-\mathrm{C}_{4} \mathrm{H}_{10} \mathrm{~N}-3\right)$ [96]; three yellow $\mathrm{PtCl}_{3}\left(\eta^{2}-\mathrm{C}_{5} \mathrm{H}_{12} \mathrm{~N}-4\right)$ [25], $\mathrm{PtCl}_{3}\left(\eta^{2}-\mathrm{C}_{5} \mathrm{H}_{12} \mathrm{~N}-2\right)$ [26] and $\mathrm{PtCl}_{3}\left(\eta^{2}-\mathrm{C}_{5} \mathrm{H}_{12} \mathrm{~N}-3\right)$ [97]. Structures of $\operatorname{Pt}\left(\eta^{2}-\text { th-4,5-ppy }\right)_{2}$ and $\operatorname{Pt}\left(\eta^{2}-\text { th-5,6-ppy }\right)_{2}$ [95] are shows in (Fig. 7). The structures of both complexes are very similar. The $\operatorname{Pt}\left(\eta^{2} \text {-th-5,6-ppy }\right)_{2}$ (Fig. 7b) has a more distorted structure caused by a stronger steric interaction of the pimene fragments. This distortion was quantified by considering the $\mathrm{Pt}-\mathrm{N}$ and $\mathrm{Pt}-\mathrm{C}$ distances as vectors and pro- jecting them into the yz plane. In $\operatorname{Pt}\left(\eta^{2}-\text { th-4,5-ppy }\right)_{2}$ complex, the (C3'- Pt - C 23') yz and (N $1-\mathrm{Pt}-\mathrm{N} 21)$ yz angles are 13.3 and $11.4^{\circ}$, respectively, whereas the same angles for $\operatorname{Pt}\left(\eta^{2}-\text { th-5,6-ppy }\right)_{2}$ are 22.5 and $18.3^{\circ}$. In both complexes the pair of heterobidentate th-4,5 ppy (in orthorhombic) and th-5,6-ppy (in hexagonal) ligands (N, C donors with two five five-membered rings create a square-planar geometry about the $\mathrm{Pt}(\mathrm{II})$ atom $\left(\mathrm{PtN}_{2} \mathrm{C}_{2}\right)$ with differing degrees of distortion. The mean $\mathrm{Pt}-\mathrm{N}$ and $\mathrm{Pt}-\mathrm{C}$ bond distances are 2.157 and $1.992 \AA$ (in orthorhombic complex); 2.170 and $1.947 \AA$ (in hexagonal complex).

There are two monoclinic $\mathrm{PtCl}_{3}\left(\eta^{2}-\mathrm{C}_{4} \mathrm{H}_{10} \mathrm{~N}-\mathrm{n}\right) \quad(\mathrm{n}=2$ [24], and 3 [96]) complexes, and three monoclinic $\mathrm{PtCl}_{3}\left(\eta^{2}\right.$ $\left.\mathrm{C}_{5} \mathrm{H}_{12} \mathrm{~N}-\mathrm{n}\right)(\mathrm{n}=4$ [25], 2 [26], and 3 [97]) which are $\pi$ - complexes. Their structures consists of discrete square - planar molecules in which the Pt(II) atom, the three chlorine atoms and the midpoint of the olefine double bond forms a plane with the platinum at the center. The mean value of $\mathrm{Pt}(\mathrm{II})-$ MP (midpoint of the double bond) is $2.05 \AA$. The mean value of $\mathrm{Pt}(\mathrm{II})-\mathrm{Cl}$ bond distance (mutually trans) of $2.307 \AA$ is about $0.023 \AA$ shorter than that of the $\mathrm{Pt}(\mathrm{II})-\mathrm{Cl}$ (trans to

Table 5. Crystallogaphic and Structural Data for Platinum Organometallic Complexes - Ligand Isomers ${ }^{\mathrm{a}}$

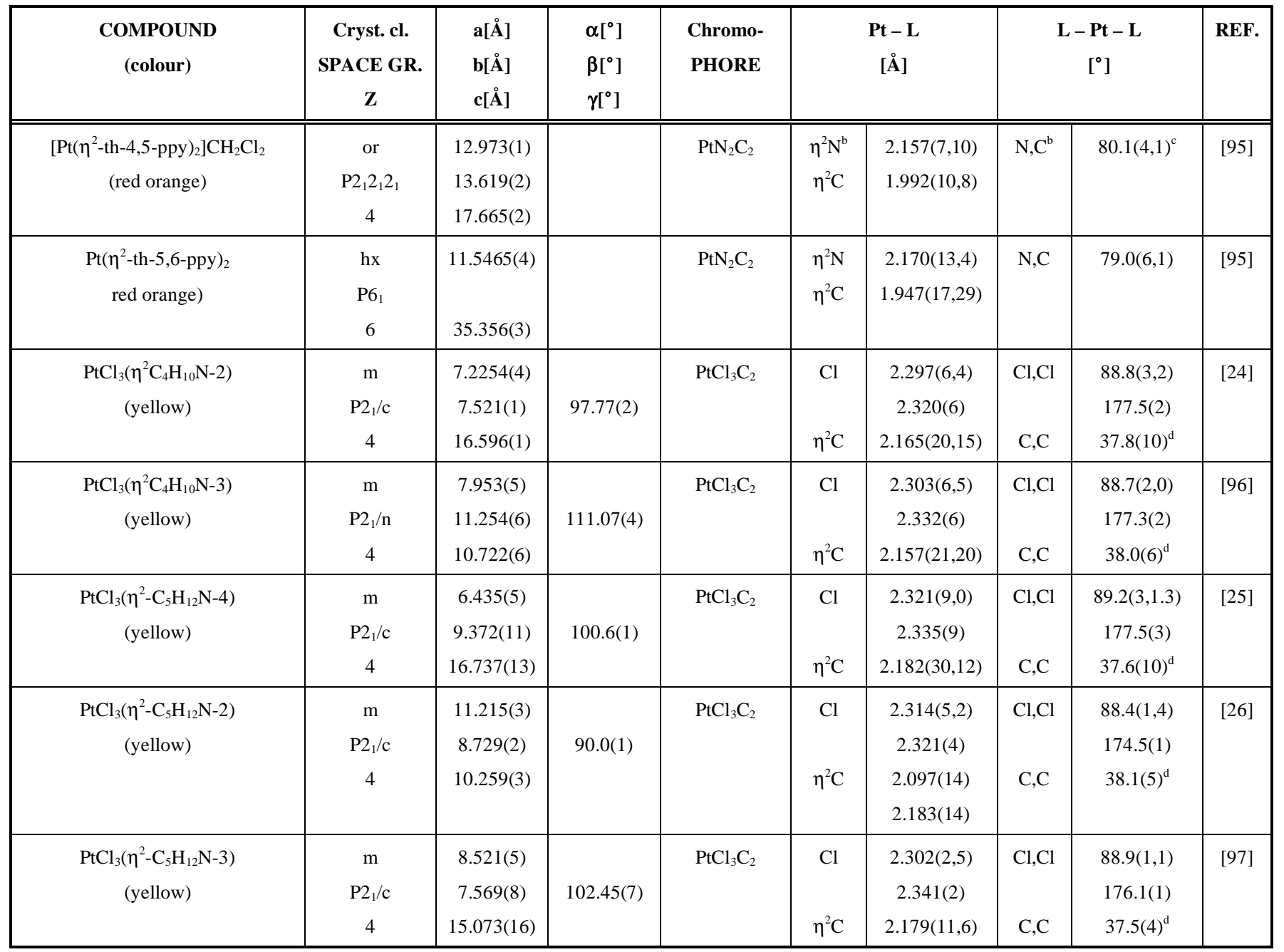

Footnotes: a). Where more than one chemically equivalent distance or angle is present, the mean value is tabulated. The first number in parenthesis is the e.s.d., and the second is the maximum deviation from the mean.

b). The chemical identity of the coordinated atom or ligand is specified in these columns.

c). Five-membered metallocyclic ring.

d). Three-membered metallocyclic ring. 

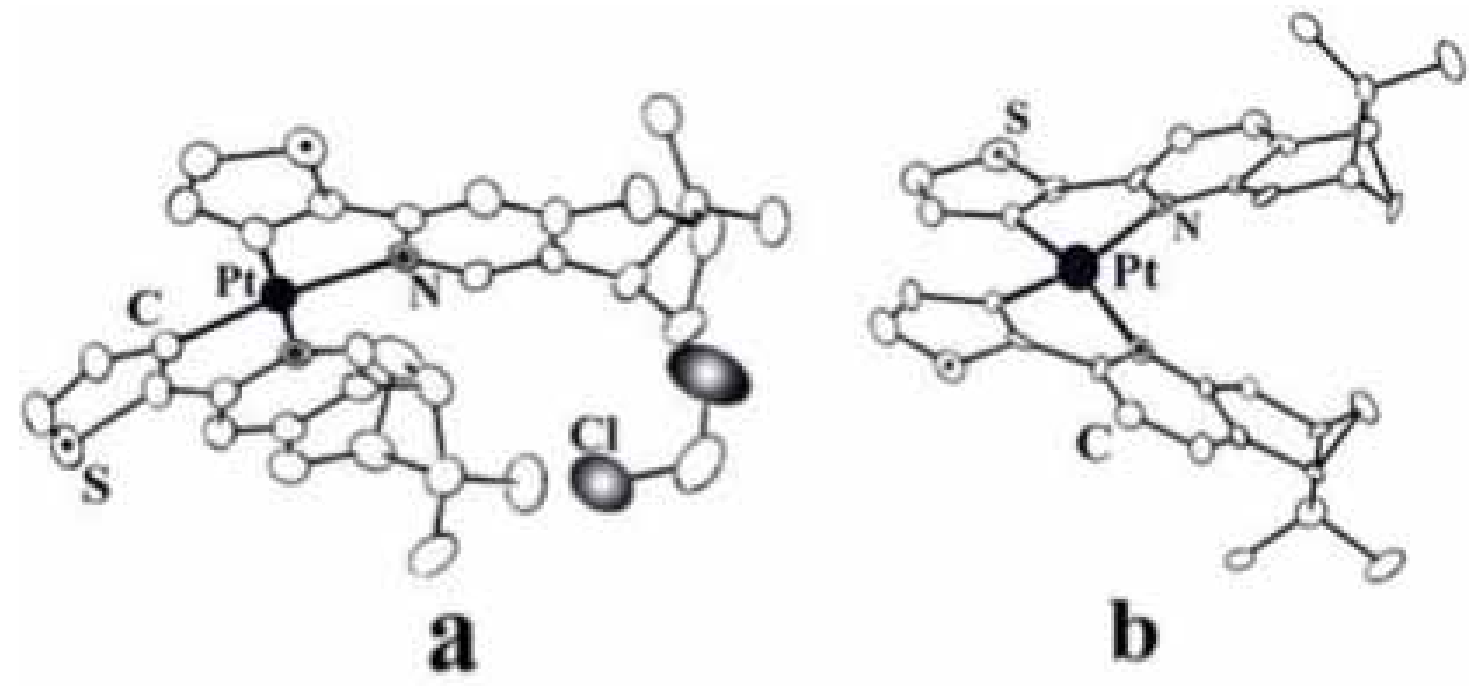

Fig. (7). Structures of $\operatorname{Pt}\left(\eta^{2} \text {-th 4,5 ppy }\right)_{2}$ (a) and $\operatorname{Pt}\left(\eta^{2} \text {-th 5,6ppy }\right)_{2}$ (b) [95].

double bond ) (2.330 ̊) clearly indicates the trans effect of the olefine double bond.

\section{CONCLUSIONS}

An analysis of almost one thousand organometallic platinum complexes shows that about $9 \%$ of them exist in isomeric forms. In general the isomers can be subdivided into two major classes, structural and stereochemical. For platinum chemistry, the latter is more prevalent. The stereoisomers found in this review can be subdivided into, distortion $(90 \%)$, cis - trans $(6 \%)$ and ligand isomers (4\%). Despite the importance of cis - trans geometry in the chemistry of $\mathrm{Pt}(\mathrm{II})$ compared to other transition metal systems, within platinum distortion isomerism is far more common. In the series of monomeric $\mathrm{Pt}$ coordination compounds where found the following types of isomerism: distortion, cistrans-, mixed and ligand, from which the distortion is by far prevails (65\%) [5].

Distortion isomers, differing only by degree of distortion in $\mathrm{Pt}-\mathrm{L}$ bond distances and $\mathrm{L}-\mathrm{Pt}-\mathrm{L}$ angles are the most numerous. They are spread over a wider range of oxidation states of platinum, (zero, $+1,+2,+4$, mixed valence +1 plus +2 and +2 plus +4 ), from which +2 is the most common. In general, in the series of distortion isomers a variations of $\mathrm{Pt}-$ $\mathrm{L}$ bond distances as well as of $\mathrm{L}-\mathrm{Pt}-\mathrm{L}$ bond angles, is depend on several factors, oxidation state of $\mathrm{Pt}(\mathrm{Pt}(\mathrm{II})$ is more favorable), soft donor atoms, (SL, PL, CL, CR and H) are most favorable, crystal packing, etc. In the series of cis-trans and ligand isomers of platinum is found only in oxidation state +2 .

The platinum atoms $\operatorname{Pt}(0)$ and $\operatorname{Pt}(\mathrm{I})$ have a tetrahedral arrangement, most $\mathrm{Pt}(\mathrm{II})$ atoms have a square planar $(\pi-$ complexes included) but some have a trigonal bipyramidal environment, and $\mathrm{Pt}(\mathrm{IV})$ atoms are found with octahedral coordination. The coordination about the platinum atom, and correlation between donor atom, bond length and interbond angles with attention to any trans effect, were noted and discussed within each section.

This review with its precursor [5] represents the first overview of the structural data of stereochemistry of plati- num complexes. Similar review of stereochemistry of heterometallic platinum clusters is in progress.

\section{ACKNOWLEDGMENTS}

The authors thank the Ministry of Education of the Slovak Republic, APVT-20-005504 and VEGA 1/2452/05 for financial support.

$$
\begin{aligned}
& \text { ABBREVIATIONS } \\
& \text { bdpep } \quad=\quad((2 \text {-(benzylthio)phenyl)azophenyl } \\
& 2 \mathrm{bchp} \quad=\text { terc-buthylcyclohexylprop-2-yn-1-ol } \\
& 3 \mathrm{bcn} \quad=\text { bicyclo[4,2,1]non-1(8)-ene } \\
& \mathrm{bdpb} \quad=1,4 \text {-bis[di(terc-butyl)phosphino]butane } \\
& \text { bpy } \quad=2,2^{\prime} \text {-bipyridyl } \\
& \text { btmsebt }=2,5 \text {-bis(trimethylsilylethynyl)bisthiophene- } \\
& \text { dyil } \\
& \text { btmset }=2,5 \text {-bis(trimethylsilylethynyl)thiophenediyl } \\
& \mathrm{Bu}^{\mathrm{t}} \quad=\text { terc-butyl } \\
& \text { bubpy }=\text { 6-terc-butyl-2,2'-bipyridinate } \\
& \text { chiraphos }=\mathrm{Ph}_{2} \mathrm{PCH}(\mathrm{Me}) \mathrm{CH}(\mathrm{Me}) \mathrm{PPh}_{2} \\
& \mathrm{C}_{4} \mathrm{H}_{8} \mathrm{O}_{2}=\text { 1,4-dioxane } \\
& \mathrm{C}_{4} \mathrm{H}_{10} \mathrm{~N}-2=\text { but-2-enylammonium } \\
& \mathrm{C}_{4} \mathrm{H}_{10} \mathrm{~N}-3=\text { but-3-enylammonium } \\
& \mathrm{C}_{4} \mathrm{H}_{12} \mathrm{~N}_{2} \quad=\text { but-2-en-1,4-diammonium } \\
& \mathrm{C}_{5} \mathrm{H}_{11} \mathrm{NMe}=\mathrm{N} \text {-methyl-4-pentenylammonium } \\
& \mathrm{C}_{5} \mathrm{H}_{12} \mathrm{~N}=\text { pent- } \mathrm{X} \text {-enylammonium }(\mathrm{X}=2,3 \text { or } 4) \\
& \mathrm{C}_{6} \mathrm{~F}_{5} \quad=\text { pentafluorophenyl } \\
& \mathrm{C}_{10} \mathrm{H}_{14}=\text { tricyclo[3,3,2.0 } \mathrm{O}^{3,7} \text { dec-3(7)-ene } \\
& \mathrm{C}_{17} \mathrm{H}_{8}=2,7 \text {-bis(ethynyl)fluorene } \\
& \mathrm{C}_{33} \mathrm{H}_{31} \mathrm{NP}_{2}=\left(\mathrm{PPh}_{2}\right) \mathrm{CH}\left(\mathrm{PPh}_{2}\right) \mathrm{CH}_{2} \mathrm{NHCH}_{2} \mathrm{Ph} \\
& \text { cod } \quad=\text { cycloocta-1,5-diene } \\
& \text { ctr }=(\text { cyclohexyl }) \mathrm{N}=\mathrm{C}\left(\mathrm{CH}_{2}\right)_{3} \mathrm{C}_{6} \mathrm{H}_{3}
\end{aligned}
$$




\begin{tabular}{|c|c|c|}
\hline dbpp & $=$ & 1,3-bis(di-terc-buthylphosphine)propane \\
\hline dmaen & $=$ & 1-[1-(dimethylamino)ethyl]-2-naphtadenyl \\
\hline dmaet & $=$ & $\mathrm{SCH}_{2} \mathrm{CH}_{2} \mathrm{NMe}_{2}$ \\
\hline dmpbpy & $=$ & $\begin{array}{l}\text { 6-(3,5-dimethylpyrazol-1-yl)-2,2'- } \\
\text { bipyridine }\end{array}$ \\
\hline dmphen & $=$ & 2,9-dimethyl-1,10-phenanthroline \\
\hline dmpm & $=$ & bis(dimethylphosphino)methane \\
\hline dmps & $=$ & $\begin{array}{l}\text { (2,2-dimethyl-4-penten-1- } \\
\text { yl)methylsulfonium methylide }\end{array}$ \\
\hline dmu & $=$ & 1,3-dimethyluracil \\
\hline dph & $=$ & 2,5-diphenyl-1,5-hexadiene \\
\hline $\mathrm{dpp}$ & $=$ & 2,9-diphenyl-1,10-phenanthrolinate \\
\hline dppe & $=$ & 1,2-bis(diphenylphosphino)ethane \\
\hline Et & $=$ & ethyl \\
\hline eox & $=$ & ethyloxazolidine \\
\hline had & $=$ & 4-homadamantyne \\
\hline hfp & $=$ & 1,1,1,3,3,3-hexafluoro-3-propanolate \\
\hline htpzb & $=$ & hydridotris(1-pyrazolyl)borate \\
\hline hxemba & $=$ & 1,5-hexadiene-d-methylbenzylamine \\
\hline ia & $=$ & 2-iodoaniline \\
\hline ipa & $=$ & isopropenylacetylide \\
\hline $\mathrm{m}$ & $=$ & monoclinic \\
\hline mbpp & $=$ & $\mathrm{C}\left(=\mathrm{CH}_{2}\right) \mathrm{CH}_{2} \mathrm{NHBu}^{\mathrm{t}}$ \\
\hline mctu & $=$ & N-methyl-N-cyanothioureate \\
\hline $\mathrm{Me}$ & $=$ & methyl \\
\hline mppe & $=$ & $\begin{array}{l}\text { 1-methyl(phenyl)phosphino-1,2- } \\
\text { diphenylethenyl }\end{array}$ \\
\hline $\mathrm{mq}$ & $=$ & 8-methylquinolyl \\
\hline msedpa & $=$ & [2-(methylsulfinyl)ethyl]diphenylarsine \\
\hline or & $=$ & orthorhombic \\
\hline ozl & $=$ & oxazolidin-2-ylidene \\
\hline $\mathrm{PBu}_{2}^{\mathrm{t}}$ & $=$ & di-terc-butylphosphinate \\
\hline $\mathrm{Pcy}_{3}$ & $=$ & tricyclohexylphosphine \\
\hline pea & $=$ & $\alpha$-phenethylamine \\
\hline $\mathrm{Ph}$ & $=$ & phenyl \\
\hline phen & $=$ & 1,10-phenanthroline \\
\hline $\mathrm{PMe}_{3}$ & $=$ & trimethylphosphine \\
\hline $\mathrm{PMePh}_{2}$ & $=$ & diphenylmethylphosphine \\
\hline Pmor $_{3}$ & $=$ & tris(morpholino)phosphine \\
\hline $\mathrm{PPh}_{3}$ & $=$ & triphenylphosphine \\
\hline $\operatorname{PPr}_{3}^{\mathrm{i}}$ & $=$ & tri-iso-propylphosphine \\
\hline ppy & $=$ & 2-phenylpyridinate \\
\hline ptpc & $=$ & 5-phenyl-1-thia-5-phosphacyclooctane \\
\hline py & $=$ & pyridine \\
\hline
\end{tabular}

\begin{tabular}{|c|c|c|}
\hline pyp & $=$ & pyridinium propylide \\
\hline pzH & $=$ & pyrazole \\
\hline rh & $=$ & rhombohedral \\
\hline salal & $=$ & salicylaldehydate \\
\hline sti & $=$ & stilbene \\
\hline af & $=$ & tetrahydrofuran \\
\hline th-4,5-ppy & $=$ & 2-(2'-thienyl)4,5-pinenopyridinate \\
\hline th-5,6-ppy & $=$ & 2-(2'-thienyl)-5,6-pinenopyridinate \\
\hline thpy & $=$ & 2-(2'-thienyl)pyridine \\
\hline tmba & $=$ & 2,2,N,N-tetramethyl-3-buten-1-amine \\
\hline tol & $=$ & toluene \\
\hline & $=$ & trigonal \\
\hline tr & $=$ & triclinic \\
\hline
\end{tabular}

\section{REFERENCES}

[1] Holloway C.E.; Melnik M. ORM I. manuscript in preparation.

[2] Holloway C.E.; Melnik M. ORM II. manuscript in preparation.

[3] Holloway C.E.; Melnik M. ORM III. manuscript in preparation.

[4] Holloway C.E.; Melnik M. ORM IV. manuscript in preparation.

[5] Melnik M.; Holloway C.E. Coord. Chem. Rev., 2006, 250, 2261.

[6] Melnik M. Coord. Chem. Rev., 1982, 47, 239.

[7] Albano V.G.; Basso Ricci G.M.; Bellon P.L. Inorg. Chem., 1969, 8,2109 .

[8] Albano V.G.; Bellon P.; Sansoni M. J. Chem. Soc., A, 1971, 2420.

[9] Dryen N.H.; Puddephatt R.J.; Roy S.; Vittal J.J. Acta Cryst., Sect. $C, \mathbf{1 9 9 4}, 50,533$.

[10] Evans J.A.; Everitt G.F.; Kenmitt R.D.W.; Russell D.R. J. Chem. Soc., Chem.Commun., 1973, 158.

[11] Cheney A.J.; MacDonald W.S.; Flynn K.O.; Shaw B.L.; Turtle B.L. J. Chem. Soc., Chem. Commun., 1973, 128

[12] Kapoor P.; Kukushkin V. Yu.; Lövquist K.; Oscarsson A. J. Organomet. Chem.,1996, 517, 71.

[13] Pandolfo L.; Paiaro G.; Ganis P.; Valle G.; Traldi P. Inorg. Chim. Acta, 1993, 200,39.

[14] Ganis P.; Paiaro G.; Pandolfo L.; Valle G. Gazz. Chim. Ital., 1990, 120,541 .

[15] Osakada K.; Kim Y.J.; Yamamoto A. J. Organomet. Chem., 1990, 382,303 .

[16] Osakada K.; Kim Y.J.; Tanaka M.; Ishiguro S.; Yamamoto A. Inorg. Chem., 1991, 30, 197.

[17] Gorla F.; Venanzi L.M.; Albinati A. Organometallics, 1994, 13, 43.

[18] Pedane C.; Benedetti E. J. Organomet. Chem., 1971, 31, 403.

[19] Fu-Yu Tsai; Ray-Hsi Hsu; Tsang-Miao Huang; Jure-Ting Chen; Gene-Hsiang Lee; Yu Wang J. Organomet. Chem., 1996, 520, 85.

[20] Tsang-Miao Huang; Jure-Ting Chen; Gene-Hsiang Lee; Yu Wang J. Am. Chem. Soc., 1993, 115, 1170.

[21] Calapietro M.; Zambonelli L. Acta Cryst., Sect. B, 1971, 27, 734.

[22] Elder R.C.; Pesa F. Acta Cryst., Sect. B, 1978, 34, 268.

[23] Spagna R.; Venanzi L.M.; Zambonelli L. Inorg. Chim. Acta, 1970, 4, 283.

[24] Spagna R.; Venanzi L.M.; Zambonelli L. Inorg. Chim. Acta, 1970, 4, 475 .

[25] Spagna R.; Zambonelli L. Acta Cryst., Sect. B, 1972, 28, 2760.

[26] Spagna R.; Ughetto G.; Zambonelli L. Acta Cryst., Sect. B, 1976, $32,2532$.

[27] Mura P.; Spagna R.; Ughetto G.; Zambonelli L. J. Cryst. Mol. Struct., 1977, 7, 265.

[28] Spagna R.; Zambonelli L. J. Chem. Soc. A, 1971, 2544.

[29] Mura P.; Spagna R.; Zambonelli L. Acta Cryst., Sect. B, 1978, 34 3745 .

[30] Bachechi F.; Zambonelli L. J. Cryst. Spect. Res., 1988, 18, 277.

[31] Benedetti E.; Carradini P.; Pedone C. J. Organomet. Chem., 1969, 18, 203; Corradini P.; Ganis P.; Pedone C. Acta Cryst., 1966, 20 458.

[32] Ganis P.; Pedone C. Rendicontia, 1965, 8, 1462 
[33] Guggolz E.; Koff M.; Ziegler M.L. Z. Anorg. Allg. Chem., 1983, 499, 59.

[34] Kopp M.; Krauth L.R.; Ratka R.; Wiedenhammer K.; Ziegler M.L. Z.Naturforsch., 1980, 35 b, 802 .

[35] Canty A.J.; Jin H.; Skelton B.W.; White A.H. Inorg. Chem., 1998, $37,3975$.

[36] Aye K.T.; Vittal J.J.; Puddephatt R.J. J. Chem. Soc., Dalton Trans., 1993, 1835.

[37] McCrindle R.; Ferguson G.; Arsnault G.J.; McAlles A.J.; Ruhl B.L.; Sneddon D.W. Organometallics, 1986, 5, 1171.

[38] Braga D.; Grepioni F.; Walther D.; Heubach K.; Schmidt A.; Imhof W.; Görls H.; Klettke T. Organometallics, 1997, 16, 4910.

[39] Jagner S.; Hazell R.G.; Rasmussen S.E. J. Chem. Soc., Dalton Trans., 1976, 337.

[40] Komatsu K.; Kamo H.; Tsui R.; Masuda H.; Takeuchi K. J. Chem. Soc., Chem.Commun., 1991, 71.

[41] Stamm E.; Becker K.B.; Engel P.; Ermer O.; Keese R. Angew. Chem. Int. Ed. Engl., 1979, 18, 685.

[42] Wicht D.K.; Zhuravel M.A.; Gregush R.V.; Glueck D.S.; Guzei I.A.; Liable-Sands L.M.; Reingold A.L. Organometallics, 1998, 17, 1412.

[43] Chin-Wing Chen; Ting-Tong Lai; Chi-Ming Che; Shie-Ming Peng J. Am. Chem. Soc., 1993, 115, 11245.

[44] Steinborn D.; Geusch M.; Bruhm C.; Davies J.A. Inorg. Chem., 1999, 38, 680 .

[45] Bonamico B.; Dessy G.; Fares V.; Russo M.V.; Scaramuzza L. Cryst. Struct.Commun., 1977, 6, 39.

[46] Nicolaides A.; SmithJ.M.; Kumar A.; Barnhart D.M.; Borden W.T. Organometallics, 1995, 14, 3475.

[47] Mole L.; Spencer J.L.; Carr N.; Orpen A.G. Organometallics, 1991, 10, 49; Carr N.; Mole L.; Orpen A.G.; Spencer J.L. J. Chem. Soc., Dalton Trans., 1992, 2653.

[48] Höpp M.; Erxleben A.; Rombeck I.; Lippert B. Inorg. Chem., 1966, 35, 397.

[49] Minghetti P.; Cinellu N.A.; Stccoro S.; Zucca A.; Manassero M. J. Chem. Soc., Dalton Trans., 1995, 777.

[50] Maasarani F.; Davidson M.F.; Wehmen-Ooyevaar I.C.M.; Grove D.M.; van Koten M.A.; Smets W.J.J.; Spek A.L.; van Koten G. Inorg. Chim. Acta, 1995, 235, 327.

[51] Pregesin P.S.; Wombacher F.; Albinati A.; Lianza F. J. Organomet. Chem., 1991, 418, 249.

[52] Casas J.M.; Falvello L.R.; Fornies J.; Martin A. Inorg. Chem., 1996, 35, 56.

[53] Johnson D.A.; Cordes A.W.; Fithen-Kelley B.A.; Deese W.C. J. Coord. Chem., 1944, 32, 1.

[54] Keeton M.; Mason R.; Russell D.R. J. Organomet. Chem., 1971, $33,259$.

[55] Robertson G.B.; Tucker P.A. Acta Cryst., Sect. C, 1983, 39, 1354.

[56] Bennett M.A.; Chee H.K.; Robertson G.B. Inorg. Chem., 1979, 18, 1061

[57] Villa A.Ch.; Manfredotti A.G.; Guastini C.; Carusi P.; Furlani A.; Russo M.V. Cryst. Struct. Commun., 1977, 6, 629.

[58] (a) Al-Juaid S.S.; Carmichael D.; Hitchcock P.B.; Kochschmidt S.; Marinetti A.; Mathey F.; Nixon J.F. J. Chem Soc., Chem. Commun., 1988, 1156; (b) Al-Juaid S.S.; Carmichael D.; Hitchcock P.B.; Marinetti A.; Mathey F.; Nixon J.F. J. Chem. Soc., Dalton Trans., 1991, 905.

[59] Briggs J.R.; Crocker Ch.; McDonald W.S.; Shaw B.L. J. Chem. Soc., Dalton Trans., 1981, 575.

[60] Balashev K.P.; Engelbretsen T.; Kwam P.I.; Martmann-Moe K.; Puzyk M.V.; Songstad J. Acta Chem. Scand., 1996, 50, 1108.

[61] Toto S.D.; Olmstead M.M.; Arbuckle B.W.; Bharadway D.K.; Musker W.K. Inorg.Chem., 1990, 29, 691.

[62] Plantevin V.; Blosser P.W.; Gallucci J.C.; Wojcicki A. Orgamnometallics, 1994, 13, 3651 .

[63] Albinati A.; Lianza F.; Berger H.; Arz C.; Pregosin P.S. Inorg. Chim. Acta., 1992, 198-200, 771.

[64] Henderson W.; Micholson B.K. Polyhedron, 1996, 15, 4015.
[65] Fanezzi F.P.; Natile G.; Lanfranchi M.; Tiripicchio A.; Pacchioni G. Inorg. Chim.Acta, 1998, 275-276, 500.

[66] Carty A.J.; Haare J.L.; Patel J.; Pfeffer M.; Skelton B.W.; White A.M. Organometallics, 1999, 8, 2660.

[67] Carty A.J.; Honeyman R.T.; Skelton B.W.; White A.M. J. Organomet. Chem., 1992, 424, 381.

[68] von Zelewsky A.; Suckling A.P.; Stoeckli-Evans H. Inorg. Chem., 1993, 32, 4585 .

[69] Gelling A.; Orrell K.G.; Osborne A.G.; Šik V.; Hursthouse B.; Coles S.J. Polyhedron, 1996, 15, 3203.

[70] McGinnety J.A. J. Organomet. Chem., 1973, 59, 429.

[71] Hassan F.S.M.; Higgins S.J.; Jacobsen G.B.; Shaw B.L.; ThorntonPett M. J. Chem. Soc., Dalton Trans., 1998, 3011.

[72] Wurst K.; Striahle J. Z. Naturforsch., 1993, 48b, 425.

[73] Chooi S.Y.M.; Ranford J.D.; Leung P.H.; Mok K.F. Tetrahedrton Assym., 1994, 5, 1805.

[74] Liu H.; Tan A.L.; Mok K.F.; Hor T.S.A. J. Chem. Soc., Dalton Trans., 1996, 4023.

[75] Baratt E.; Boudreaux E.A.; Demas J.N.; Lenhert P.G.; Lukehart C.M.; McPhail A.T.; McPhail D.R.; Myers J.B. Jr.; Sacksteder L.; True W.R. Organometallics, 1989, 8, 2417.

[76] Jans J.; Naegeli R.; Venani L.M.; Albinati A. J. Organomet. Chem., 1983, 247, C37.

[77] (a) Lydon J.E.; Truter M.R.; Watling R.C. Proc. Chem Soc. (London), 1964, 193; (b) Truter M.R.; Watling R.C. J. Chem. Soc. A, 1967, 1955; (c) Lydon J.E.; Truter M.R. ibid, 1965, 6899.

[78] Park S.; Roundhill D.M.; Rheingold A.L. Inorg. Chem., 1987, 26, 3972; Organometallics, 1991, 10, 615 .

[79] Bertani R.; Mozzon M.; Michelin R.A.; Benetollo F.; Bombieri G.; Castilhí T.J.; Pombieno A.J.L. Inorg. Chim. Acta, 1991, 189, 175.

[80] Manojlovi-Muir L.; Muir K.W.; Frew A.A.; Ling S.S.M.; Thompson M.A.; Puddhephatt R.J. Organometallics, 1984, 3, 1637; Preddephatt R.J.; Thomson A.A.; Manojlovi-Muir L.; Muir K.W.; Frew A.A.; Brown M.P. J. Chem. Soc., Chem. Commun., 1981, 805.

[81] Albinati A.; Chaloupka S.; Eckert J.; Venanzi L.M.; Wolfer M.K. Inorg. Chim. Acta, 1997, 259, 305.

[82] Lewis J.; Long N.J.; Raitby P.R.; Shields G.P.; Wong W.Y.; Younus M. J. Chem. Soc., Dalton Trans., 1997, 4283.

[83] Wong W.Y.; Wong W.K.; Raithby P.R. J. Chem. Soc., Dalton Trans., 1998, 2761.

[84] Bruno R.A.; Farrar D.H.; Irwin J.J. Inorg. Chim. Acta, 1991, 181 65 .

[85] Leoni P.; Manetti S.; Pascquali M.; Albinati A. Inorg. Chem., 1996, 35,6045

[86] Duran M.; Clegg W.; Fraser K.A.; Gonzalez-Duarte P. Inorg. Chim. Acta, 2000, 300-302, 790.

[87] Klevstsova R.F.; Yurchenko E.N.; Glinskaya L.A.; Burgina E.B.; Eremenko N.K.; Bkakin V.V. Zh. Strukt. Khim., 1985, 26, 84; Engl. Ed., p. 216.

[88] Ebert K.H.; Massa W.; Donath H.; Lorberth J.; Seo B.S.; Herdtweck E. J. Organomet. Chem., 1998, 559, 203.

[89] Raper G.; McDonald W.S. J. Chem. Soc., Dalton Trans., 1972, 265.

[90] Kernbach U.; Fehlhammer W.P. Inorg. Chim. Acta, 1995, 235, 299.

[91] Stang P.J.; Kowalski M.H. J. Am. Chem. Soc., 1989, 111, 3356.

[92] Chen J.T.;. Yeh Y.S; Yang Ch.S.; Tsai F.Y.; Huang G.L.; Shu B.Ch.; Huang T.M.; Chen Y.S.; Lee G.H.; Cheng M.Ch.; Wang Ch.Ch.; Wang Y. Organometallics, 1994, 43, 4804.

[93] Manojlovi-Muir L.; Muir K.W. J. Chem. Soc., Dalton Trans., 1974, 2427.

[94] Lin I.J.B.; Kao L.T.C.; Wu F.J.; Lee G.H.; Wang Y. J. Organomet. Chem., 1986, 309, 225.

[95] Gianini M.; Ferster A.; Haang P.; von Zelewski A.; Stoeckli-Evans H. Inorg. Chem., 1996, 35, 4889.

[96] Mura P.; Spagna R.; Zambonelli L. J. Organomet. Chem., 1977, 142, 403.

[97] Mura P.; Spagna R.; Zambonelli L. Acta Cryst., Sect. B, 1976, 32, 2532. 